\title{
Autoionizing states of atomic boron
}

\author{
Luca Argenti ${ }^{1, *}$ and Roberto Moccia ${ }^{2}$ \\ ${ }^{1}$ Departamento de Química, Módulo 13, Universidad Autónoma de Madrid, 28049 Madrid, Spain, EU \\ ${ }^{2}$ IPCF, CNR, Via Giuseppe Moruzzi 1, 56100 Pisa, Italy, EU
}

(Received 1 February 2016; published 8 April 2016)

\begin{abstract}
We present a $B$-spline $K$-matrix method for three-active-electron atoms in the presence of a polarizable core, with which it is possible to compute multichannel single-ionization scattering states with good accuracy. We illustrate the capabilities of the method by computing the parameters of several autoionizing states of the boron atom, with ${ }^{2} S^{e},{ }^{2} P^{e, o}$, and ${ }^{2} D^{e}$ symmetry, up to at least the $2 p^{2}\left({ }^{1} S\right)$ excitation threshold of the B II parent ion, as well as selected portions of the photoionization cross section from the ground state. Our results exhibit remarkable gauge consistency, they significantly extend the existing sparse record of data for the boron atom, and they are in good agreement with the few experimental and theoretical data available in the literature. These results open the way to extend to three-active-electron systems the spectral analysis of correlated wave packets in terms of accurate scattering states that has already been demonstrated for two-electron atoms in Argenti and Lindroth [Phys. Rev. Lett. 105, 053002 (2010)].
\end{abstract}

DOI: 10.1103/PhysRevA.93.042503

\section{INTRODUCTION}

Accurate records for photoexcitation and photoionization atomic spectra, obtained either from measurements or from state-of-the-art calculations, are instrumental in monitoring the properties of matter in extreme conditions, such as hightemperature plasma in laboratory [1], in stellar coronas [2,3], and in black-hole accretion disks [4,5] or the rarefied gases in galactic halos [6] and in the intergalactic medium [7], which are traversed by ionizing radiation [8,9]. Such atomic records are also essential to test new models for correlated electron dynamics in finite many-body systems.

On the experimental side, information on atomic spectra has been initially provided by classical spectroscopy and subsequently by photoionization experiments conducted with synchrotron radiation $[10,11]$. Thanks to the technical advancements in the synchronization of light pulses [12] and in the detection methods for the charged fragments that emerge from the interaction region, the energy resolution of measurements conducted at synchrotron facilities has steadily increased [13-15]. Measurements of the autoionizing series in rare gases with a resolving power as high as $10^{6}$ were recently reported [16]. Velocity-map imaging apparatuses [17] simplified the measurement of angularly resolved photoelectron spectra, while multiple detection techniques such as COLTRIMS [18] permit to do so for several charged fragments in coincidence. Furthermore, using lasers in association with synchrotron beamlines, it is possible to measure photoabsorption spectra from excited states $[19,20]$. Recently, the study of photoionization processes has gained new momentum thanks to the advent of attosecond light sources [21], which opened the way to the time-resolved study of electron photoemission and of correlated electron dynamics in atoms and molecules at its natural time scale [22-24].

Most experimental measurements, however, concern rare gases or highly volatile elements. For other elements, which still represent a challenge due to the difficulty of preparing

\footnotetext{
*luca.argenti@uam.es
}

gaseous samples with sufficient optical density, theoretical calculations are a convenient alternative. In fact, computational methods are now able to provide theoretical spectra with an accuracy that is competitive with experiment [25]. Widely used codes for atomic photoionization implement the $R$-matrix method [26]. The Belfast atomic codes [27], in particular, have been used to compile extensive databases of spectral data of astrophysical interest, such as the opacity project [28-32] and the Chianti project [33,34]. The $B$-spline $R$ matrix code [35-37], a more recent example of this approach, has already been used to accurately reproduce several fully differential collisional and photoionization observables [38]. An alternative general-purpose atomic package, which makes use of exterior complex scaling instead of the $R$-matrix method, and which is based on a $B$-spline close-coupling (CC) extension of the multiconfiguration Hartree-Fock (MCHF) Fröse-Fischer's ATSP2K libraries [39,40], is currently under development in Stockholm [41].

The literature now reports theoretical discrete transition probabilities and photoionization cross sections for most of the light atoms of the second period, as well as for some of their ions. In the case of a neutral boron atom, however, the photoionization cross sections record is still rather scant. Various studies examined in detail the ground-state energy, the discrete spectrum [42-59], the electron affinity [60,61], the electron impact excitation and ionization [62-66], and the $1 s$ core excitation [67] and core photoionization [68,69] of the boron atom. Discrete and photoionization spectra of the $\mathrm{B}^{+}$parent ion have also been computed [70-75]. To date, however, we are aware of only two sources for the theoretical photoionization spectrum of boron from the valence shell: a work of the multichannel photoionization cross sections up to $\sim 100 \mathrm{eV}$ above threshold [76], which only accounts for the background signal, without reporting any resonant or other high-resolution feature of the spectrum, and the opacity project [32], which focuses on the high end of the energy spectrum and only provides total photoionization cross sections on an energy grid too sparse to resolve any resonant structure.

Part of the reason why the photoionization of boron failed to attract larger interest is possibly its peculiar nucleogenesis, 
which takes place through spallation of heavier elements in the interstellar medium [77] rather than by nuclear fusion in a stellar interior, where, in fact, it is consumed [78]. Furthermore, boron's most characteristic spectral features, at 209 and $250 \mathrm{~nm}$ [79], are not easily observable from Earth, requiring an orbiting telescope instead. Since the recent commissioning of a UV spectrometer on the Hubble telescope, however, the situation may change, with boron becoming more relevant for diagnostics of astrophysical processes [78]. Boron is interesting also from a purely theoretical perspective, since it is the simplest atomic system that features already in its ground state three valence electrons with similar binding energies and thus arguably strongly interacting with each other. In comparison with two-active-electron atoms, which have been the subject of a staggering amount of theoretical studies [80], the continuum of three-active-electron systems is still, on the whole, a scantly explored territory [81]. Indeed, the large size of the configuration space hampers calculations in the continuum at a full-configuration-interaction (full-CI) level.

In this work we contribute to fill this gap by presenting comprehensive sets of parameters (position, width, branching ratios) for the autoionizing states of the neutral boron atom with ${ }^{2} S^{e},{ }^{2} P^{e, o}$, and ${ }^{2} D^{e}$ symmetry, within $\approx 16 \mathrm{eV}$ from the first ionization threshold, computed with the $B$-splines $K$-matrix method [82]. Within the autoionizing series, several intruder states appear. We also present few selected portions of the photoionization cross sections of the atom from the ${ }^{2} P^{o}$ ground state, as a way of discriminating between resonances with different character. Good gauge invariance of the cross sections indicates that the present results are well converged. This is also confirmed by the agreement between the results of the present work and the few theoretical and experimental data available in the literature. Finally, explicit calculation of scattering states for two-electron systems has proved useful to analyze the asymptotic properties of the correlated electron wave packets generated in time-resolved simulations of photoionization events triggered by femtosecond and attosecond light pulses [83-88]. This work demonstrates that such approach can be extended to more complex systems, for which the representation of scattering states in terms of orthogonal single partial-wave channels is not possible, due to the correlated character of the parent ions, thus laying the foundations for a time-resolved study of the correlated motion of three electrons in the continuum.

The paper is organized as follows. Section II summarizes the $K$-matrix method and illustrates its implementation for a three-active-electron atom with a polarizable core. Section III describes the details of the calculation for the boron atom, presents bound-state energies and resonance parameters, and compares them with the theoretical and experimental values available in the literature. Finally, Sec. IV draws the conclusions.

\section{THEORETICAL METHOD}

In the $K$-matrix method, the multichannel single-ionization scattering states at a given energy $E$ of an $N$-electron atom are sought as solutions of the Lippmann-Schwinger equation in stationary form [89], fulfilling assigned boundary conditions,

$$
\left|\Psi_{\alpha E}^{\mathcal{P}}\right\rangle=\left|\phi_{\alpha E}\right\rangle+\sum_{\gamma} \bigvee d \epsilon\left|\phi_{\gamma \epsilon}\right\rangle \frac{\mathcal{P}}{E-\epsilon} K_{\gamma \epsilon, \alpha E} .
$$

In this expression, $\left|\phi_{\gamma_{E}}\right\rangle$ are partial-wave channel (PWC) functions defining ionization states, in the LS-coupling approximation, in which the coupled parent ion and free electron have well-defined asymptotic quantum numbers. The PWCs are defined as

$$
\begin{aligned}
\phi_{\alpha E}\left(x_{1}, \ldots, x_{N}\right)= & \hat{\mathcal{A}} \sum_{M_{a} m} \sum_{\Sigma_{a} \sigma} C_{L_{a} M_{a}, \ell m}^{L M} C_{S_{a} \Sigma_{a}, \frac{1}{2} \sigma}^{S \Sigma} \Phi_{a, M_{a} \Sigma_{a}} \\
& \times\left(x_{1}, \ldots, x_{N-1}\right)^{2} \chi_{\sigma}\left(\zeta_{N}\right) Y_{\ell m}\left(\hat{r}_{N}\right) \frac{f_{\alpha, \epsilon}\left(r_{N}\right)}{r_{N}},
\end{aligned}
$$

where an eigenstate $\Phi_{a}$ of the $(N-1)$-electron parent ion, $H_{N-1}\left|\Phi_{a}\right\rangle=\left|\Phi_{a}\right\rangle E_{a}$, target state, with angular momentum $L_{a}$ and spin $S_{a}$, is coupled to an electron with well-defined asymptotic energy $\epsilon=E-E_{a}$ and angular momentum $\ell$. In the previous expression, $\hat{\mathcal{A}}$ is the $N$-electron antisymmetrizer, $C_{\ell_{1} m_{1}, \ell_{2} m_{2}}^{\ell_{12} m_{12}}$ are Clebsch-Gordan coefficients, $Y_{\ell m}(\hat{r})$ are spherical harmonics, the variable $x_{i} \equiv\left(\vec{r}_{i}, \zeta_{i}\right)$ indicates the pair of spatial and spin coordinates for the $i$ th electron, while lowercase greek indexes represent collectively all the total quantum numbers, besides energy, needed to fully characterize the state asymptotically. These comprise, on the one hand, the good total quantum numbers, such as the angular momentum $L$, its projection $M$, the total spin $S$, its projection $\Sigma$, and parity $\pi$, which identify the total symmetry of the system, indicated here as $\Gamma=(S, L, \pi, \Sigma, M)$, and, on the other hand, the (unoriented) state $a$ of the parent ion and the orbital angular momentum $\ell$ of the $N$ th electron, $\alpha=(\Gamma ; a, \ell)$. In Eq. (1), $\gamma$ runs over all channels within an assigned symmetry $\Gamma$. The CC expansion coefficients in (1), which are expressed in terms of the off-shell reaction matrix $K_{\gamma \epsilon, \alpha E}$, are uniquely determined by requiring $\left|\Psi_{\alpha E}^{\mathcal{P}}\right\rangle$ to be an eigenstate of the total Hamiltonian $H,(H-E)\left|\Psi_{\alpha E}^{\mathcal{P}}\right\rangle=0$. When projected on a complete set of $L^{2}$ basis functions, the time-independent Schrödinger equation for the continuum provides a system of linear integral equations for $K_{\gamma \epsilon, \alpha E}$, which can be discretized and solved with standard numerical methods. It should be noticed that in the $K$-matrix method, in contrast to Feshbach formalism [90], PWCs are not required to be orthogonal, their possible overlap being consistently taken into account in the $K$-matrix equations. To improve convergence, the $\mathrm{CC}$ basis is supplemented with a set of $N$-electron functions, built from orbitals localized in the vicinity of the nucleus, that accounts for short-range electron correlation and which is called here the localized channel (LC). The numerical technique employed to solve the $K$-matrix equations in the extended $\mathrm{CC}$ space defined by the PWCs and the LC is fully described in previous publications ([82,91-94]) and will not be repeated here. Once the expansion coefficients in (1) have been determined, the on-shell reaction matrix, $\mathbf{K}(E)$, is obtained interpolating $K_{\alpha \epsilon, \beta E}$ on the energy shell, $K_{\alpha \beta}(E)=K_{\alpha E, \beta E}$. The solutions $\left|\Psi_{E}^{\mathcal{P}}\right\rangle=\left(\left|\Psi_{\alpha E}^{\mathcal{P}}\right\rangle,\left|\Psi_{\beta E}^{\mathcal{P}}\right\rangle, \ldots\right)$, for all open channels, form a complete, nonorthogonal set of stationary scattering states at energy $E,\left\langle\boldsymbol{\Psi}_{E^{\prime}}^{\mathcal{P}} \mid \boldsymbol{\Psi}_{E}^{\mathcal{P}}\right\rangle=\delta\left(E-E^{\prime}\right)\left[1+\pi^{2} \mathbf{K}^{2}(E)\right]$. The 
scattering states $\left|\Psi_{E}^{ \pm}\right\rangle=\left(\left|\Psi_{\alpha, E}^{ \pm}\right\rangle,\left|\Psi_{\beta, E}^{ \pm}\right\rangle, \ldots\right)$ that fulfill outgoing $(+)$ or incoming $(-)$ boundary conditions are obtained from the stationary solutions $\left|\Psi_{E}^{\mathcal{P}}\right\rangle$ through the transformation $\left|\boldsymbol{\Psi}_{E}^{ \pm}\right\rangle=\left|\boldsymbol{\Psi}_{E}^{\mathcal{P}}\right\rangle /[1 \pm i \pi \mathbf{K}(E)]$, so that the scattering matrix $\mathbf{S}(E)$ can be computed as

$$
\mathbf{S}(E)=[1-i \pi \mathbf{K}(E)][1+i \pi \mathbf{K}(E)]^{-1} .
$$

The scattering matrix is unitary and, for time-reversal invariant systems such as those examined here, it is also symmetric. Therefore, it can be expressed as $\mathbf{S}(E)=$ $\mathbf{O}(E) \exp [2 i \varphi(E)] \mathbf{O}^{t}(E)$, where $\left\{\varphi_{\alpha}(E)\right\}$ are the so-called eigenphases, whereas the real orthogonal vectors $\left\{\mathbf{O}_{\bullet \alpha}(E)\right\}$ are the corresponding eigenchannels. Resonances are assumed to be the rank 1 poles of the scattering matrix. In particular, they are simple poles of its determinant $|\mathbf{S}(E)|$. Their position $\tilde{E}_{a}=E_{a}-i \Gamma_{a} / 2$, therefore, can conveniently be determined by fitting $|\mathbf{S}(E)|$ with products of unitary Breit-Wigner factors, $|\mathbf{S}(E)|=\exp \left[2 i \varphi_{\mathrm{bg}}(E)\right] \prod_{a}(E-$ $\left.\tilde{E}_{a}^{*}\right) /\left(E-\tilde{E}_{a}\right)$, where $\varphi_{\mathrm{bg}}(E)$ is a nonresonant background phase. Equivalently, one can directly fit the total phase shift $\varphi_{\text {tot }}(E)=\frac{1}{2} \arg |\mathbf{S}(E)|$ as $\varphi_{\text {tot }}(E)=\varphi_{\text {bg }}(E)+\sum_{a}\{\pi / 2+$ $\left.\arctan \left[2\left(E-E_{a}\right) / \Gamma_{a}\right]\right\}$. In this work, we follow this second approach, which is well established and proved able to yield robust results [95]. Apart for their position $E_{a}$ and width $\Gamma_{a}$, autoionizing states are also characterized by the partial widths $\Gamma_{\alpha, a}$, with which they decay to individual open channels. In principle, the partial widths $\Gamma_{\alpha, a}$ can be obtained from the residuals of the scattering matrix at the pole $\tilde{E}_{a}$ [96], $\Gamma_{\alpha, a}=i \lim _{z \rightarrow \tilde{E}_{a}}\left(z-\tilde{E}_{a}\right) S_{\alpha \alpha}(z)$. The analytic continuation to the complex plane of the full matrix $\left(E-\tilde{E}_{a}\right) \mathbf{S}(E)$, however, is numerically problematic. Here, therefore, we follow an alternative approximate procedure. In the vicinity of an isolated resonance, the scattering matrix can be represented as $\mathbf{S}(E)=\mathbf{S}_{\mathrm{bg}}(E)-i \boldsymbol{\gamma}_{a} \boldsymbol{\gamma}_{a}^{t} /\left(E-\tilde{E}_{a}\right)$ [96], where $\boldsymbol{\gamma}_{a}$ is the vector of partial-width amplitudes, $\Gamma_{\alpha, a}=\left|\gamma_{\alpha, a}\right|^{2}$. By taking the derivative at $E_{a}$, one finds $\mathbf{S}^{\prime}\left(E_{a}\right)=\mathbf{S}_{\mathrm{bg}}^{\prime}\left(E_{a}\right)-i \boldsymbol{\gamma}_{a} \boldsymbol{\gamma}_{a}^{t} / \Gamma_{a}^{2}$. If the background changes slowly compared with the resonant term, then the largest eigenvalue of $\left|\mathbf{S}^{\prime}\left(E_{a}\right)\right|^{2}$ is very close to $16 / \Gamma_{a}^{2}$, and the corresponding eigenvector $\boldsymbol{u}_{a}$ is a good estimator of the partial-width amplitudes.

\section{A. Polarizable-core model}

In this work we are interested in the autoionizing states of boron at energies much smaller than that required to excite an electron out of the $1 s^{2}$ core, which is of the order of 10 a.u. Under these conditions, it is convenient to consider as active only the three valence electrons, whereas the core is treated as a frozen single-determinant shell. To make theoretical predictions that are accurate enough to be compared with the experiment, nevertheless, it is still important to account for the dynamical correlation between core and valence electrons. A large part of this correlation manifests itself as a polarization of the core electrons in response to the electrostatic field generated by the electrons in the valence shell. A convenient way to account for this effect is to include in the corevalence interaction Hamiltonian, beyond the usual Coulomb and exchange terms, a regularized semiempirical corepolarization potential given by the sum of a one-particle and a two-particle effective component, $V_{\mathrm{p}}=V_{\mathrm{p}}^{(1)}+V_{\mathrm{p}}^{(2)}$, defined as [97]

$$
\begin{gathered}
V_{\mathrm{p}}^{(1)}=-\frac{\alpha_{\mathrm{c}}}{2} \sum_{i \ell} g\left(r_{i}, R_{\ell}\right) \pi_{\ell}(i), \\
V_{\mathrm{p}}^{(2)}=-\frac{\alpha_{\mathrm{c}}}{2} \sum_{i j} \sum_{\ell_{1}-\ell_{4}} \sqrt{g\left(r_{i}, \bar{R}_{\ell_{1} \ell_{3}}\right) g\left(r_{j}, \bar{R}_{\ell_{2} \ell_{4}}\right)} \\
\times \pi_{\ell_{1}}(i) \pi_{\ell_{2}}(j) \hat{r}_{i} \cdot \hat{r}_{j} \pi_{\ell_{3}}(i) \pi_{\ell_{4}}(j),
\end{gathered}
$$

where $\alpha_{\mathrm{c}}$ is the core static polarizability, $\pi_{\ell}(i)=\sum_{\mu}|\ell \mu\rangle\langle\ell \mu|$ is the projector on the subspace with orbital angular momentum $\ell$, for electron $i$, and $g(r, R)=r^{-4}\left\{1-\exp \left[(r / R)^{6}\right]\right\}$ is a regularized polarization potential that smoothly turns off when the electron penetrates the core region, where the static-polarization model is not applicable. The $\ell$-dependent cutoff radii $R_{\ell}$, beyond which the polarization interaction is switched on, are adjustable parameters of the model. In the two-electron term, the average $\bar{R}_{\ell \ell^{\prime}}=\left(R_{\ell}+R_{\ell^{\prime}}\right) / 2$ is used. The core-valence polarization potential (4), (5) is known to reproduce well the effects of long-range core-valence electron correlation [98-102] and to give results equivalent to those obtained with more rigorous approaches [103]. In the case of boron, in particular, due to the clear separation between the spatial distribution of core and valence electrons, both the switch off of the polarization potential and the orthogonality constraint are well justified. In this work, the core static polarizability, $\alpha_{\mathrm{c}}=0.0193$ a.u., is computed as $\alpha_{\mathrm{c}}=2 \sum_{n}\left|\left\langle g{ }^{1} \mathrm{~S}\left|z_{1}+z_{2}\right| n^{1} \mathrm{P}^{o}\right\rangle\right|^{2} /\left(E_{n}^{1} \mathrm{P}^{o}-E_{g}{ }^{1} \mathrm{~S}\right)$, where the eigenstates and energies of $\mathrm{B}^{3+}$ ( $g$ is the $\sim 1 s^{2}$ ground state) are obtained from a full-CI calculation with maximum orbital angular momentum $\ell=4$. The cutoff parameters $R_{\ell}=$ 0.7 a.u., for $\ell \neq 1$, and $R_{1}=0.71$ a.u., give excitation energies for the $\mathrm{B}^{2+}$ ion in good agreement with the experiment. In the polarizable frozen-core model, the states of the boron atom or ion are represented as functions of valence electrons only, with single-particle factors orthogonal to the $1 \mathrm{~s}$ core orbital, governed by the effective Hamiltonian

$$
H_{\mathrm{eff}}=E_{\mathrm{c}}+H+J_{\mathrm{c}}-K_{\mathrm{c}}+V_{\mathrm{p}},
$$

where $H$ is the electrostatic Hamiltonian of either the $\mathrm{B}^{2+}$ or the $\mathrm{B}^{3+}$ ion, whereas $J_{\mathrm{c}}$ and $K_{\mathrm{c}}$ are the Coulomb and exchange operator due to the $1 s^{2}$ core. Neglecting core correlation does not have any major impact on the relative energies of valenceexcited states.

\section{B. Three-electron close-coupling states}

In the $B$-spline $K$-matrix method, all the radial parts of the electron orbitals, and in particular the reduced radial wave function of the $N$ th electron, are expanded in terms of a set of $B$ splines [82,104,105] defined in a quantization box. To achieve satisfactory accuracy while keeping the configuration space within manageable proportions, we use two different sets of $B$ splines: a localized one, $\left\{B_{i}^{\text {loc }}\right\}$, defined by a series of knots with increasing spacing and spanning a radial range up to a few tens of Bohr radii, to describe interacting electrons bound at short range, and a diffuse one, $\left\{B_{i}^{\text {wav }}\right\}$, with constant spacing up to large distances, which can exhibit rapid oscillations and describe asymptotically free electrons. To build the two-electron basis functions for 
the parent ion, as well as the three-electron LC, we first solve the Hartree-Fock equations for the neutral atom in the space defined by the localized $B$ splines, thus obtaining a set of self-consistent-field (SCF) orbitals $\phi_{\ell m n}(\vec{r})=$ $\phi_{\ell n}(r) Y_{\ell m}(\hat{r}), \phi_{\ell n}(r)=\sum_{i} B_{i}^{\text {loc }}(r) r^{-1} c_{i, \ell n}$. The two-electron bases with symmetry $\Gamma_{a}=\left(S_{a}, L_{a}, \pi_{a}, \Sigma_{a}, M_{a}\right)$ are constructed from the SCF orbitals as

$$
\phi_{\ell_{1} \ell_{2} n_{1} n_{2}}^{\Gamma}=\Theta_{S_{a} \Sigma_{a}}\left[1(-)^{S_{a}} \sigma_{12}\right]\left[\phi_{\ell_{1} n_{1}} \otimes \phi_{\ell_{2} n_{2}}\right]_{L_{a} M_{a}},
$$

where $\Theta_{S_{a} \Sigma_{a}}$ is a singlet or triplet spin function, $\sigma_{12}$ is the permutation of the two electrons, and we used the notation $\left[\mathcal{M}_{a} \otimes \mathcal{N}_{b}\right]_{c \gamma} \equiv \sum_{\alpha \beta} C_{a \alpha, b \beta}^{c \gamma} \mathcal{M}_{a \alpha} \mathcal{N}_{b \beta}$ for the coupling of irreducible spherical tensors. Diagonalization of the Hamiltonian (6) in a two-electron full-CI basis, $H_{\mathrm{eff}} \Phi_{a}=E_{a} \Phi_{a}$, provides accurate target states $\Phi_{a}$, which are exactly factorizable as $\Phi_{a}=\Theta_{S_{a} \Sigma_{a}} F_{a}$. In this case, the PWC (2) can be written in the compact form

$$
\phi_{\alpha n}=\hat{\mathcal{A}} \Theta_{S \Sigma ; S_{a}}\left[F_{a} \otimes \varphi_{n \ell}\right]_{L M},
$$

where $\Theta_{S \Sigma ; S_{a}}=\left[\Theta_{S_{a}} \otimes{ }^{2} \chi\right]_{S \Sigma}$ and $\left[F_{a} \otimes \varphi_{n \ell}\right]_{L M}$ are the coupled spin and spatial components of the parent ion with the third electron, whose radial part is expressed in the diffuse $B$-spline basis. The spin components for a given $S, \Theta_{S \Sigma ; S_{a}}$ belong to the irreducible representation $\gamma_{S}$ of the $\mathcal{S}_{3}$ symmetric group, with $\gamma_{\frac{1}{2}}=\{2,1\}$ and $\gamma_{\frac{3}{2}}=\{3\}$ [106]: for $\sigma \in \mathcal{S}_{3}, \sigma \Theta_{S \Sigma ; \tau}=\sum_{\zeta} \Theta_{S \Sigma ; \zeta} D_{\zeta \tau}^{\gamma_{s}}(\sigma)$. Using group theory, it is possible to evaluate the effect of $\hat{\mathcal{A}}$ on the spin functions in Eq. (7), obtaining the expression

$$
\phi_{\alpha n}=n_{\gamma_{s}}^{-1} \sum_{\varsigma} \Theta_{S \Sigma ; \zeta} \hat{\rho}_{\varsigma S_{a}}^{\bar{\gamma}_{s}}\left[F_{a} \otimes \varphi_{n \ell}\right]_{L M},
$$

where $\hat{\rho}_{\zeta \tau}^{\bar{\gamma}_{s}}=\frac{n_{\gamma_{s}}}{3 !} \sum_{\sigma \in \mathcal{S}_{3}} \operatorname{sgn} \sigma D_{\varsigma \tau}^{\gamma_{s}}(\sigma) \sigma$ is the Wigner operator for the dual representation of $\gamma_{s}$ [107], $D_{\varsigma \tau}^{\bar{\gamma}_{s}}(\sigma)=$ $\operatorname{sgn} \sigma D_{\varsigma \tau}^{\gamma_{s} *}(\sigma)$, with $n_{\frac{1}{2}}=2$ and $n_{\frac{3}{2}}=1$. From Eq. (8) it is easy to derive the expression for the reduced matrix elements of any spin-free operator $O$ symmetric under permutations,

$$
\left\langle\phi_{\alpha i}\|O\| \phi_{\beta j}\right\rangle=n_{\gamma_{s}}^{-1}\left\langle\left[F_{a} \otimes \varphi_{i \ell_{\alpha}}\right]_{L_{\alpha}}\left\|O \hat{\rho}_{S_{a} S_{b}}^{\bar{\gamma}_{s}}\right\|\left[F_{b} \otimes \varphi_{j \ell_{\beta}}\right]_{L_{\beta}}\right\rangle .
$$

This formulation, which does not assume orthogonality between single-particle wave functions, permits us to take advantage of the sparsity of the primitive matrix elements built using the $B$-spline radial basis. The angular part of the spatial integrals in the last expression can be computed using standard techniques of angular momentum algebra. In the Appendix we report the contraction formulas for arbitrary one-body tensor operators and for scalar two-body operators.

The diagonalization of the Hamiltonian (6) projected on a given PWC yields a Rydberg series of discrete states with energy below that of the PWC parent ion and a set of states with energy above that of the parent ion, which provide a discretized representation for the continuum states of an asymptotically free electron in the field of the parent ion. When the PWCs are allowed to interact, within any assigned total symmetry, the Rydberg series converging to the parent ion with the lowest energy, among those available, form the backbone of the bound states of the neutral atom in that symmetry, while the Rydberg series bound to more excited parent ions gives rise to autoionizing states that decay to the channels with lower thresholds by emitting an electron.

Since the CC approach already describes well the electronic correlation of the $\mathrm{B}^{+}+e^{-}$pair at intermediate distances, the LC configurations must only account for the residual short-range correlation, and are thus built using localized orbitals only,

$$
\phi_{\ell n, s_{12} \ell_{12}}^{\Gamma}=\hat{\mathcal{A}} \Theta_{S \Sigma, S_{12}}\left[\left[\phi_{n_{1} \ell_{1}} \otimes \phi_{\ell_{2} n_{2}}\right]_{\ell_{12}} \otimes \phi_{\ell_{3} n_{3}}\right]_{L M} .
$$

Even with this restriction, however, the full-CI three-electron space is still too large to be entirely included in the LC. Luckily, a comparatively small number of configurations can already improve significantly the results obtained with the PWC CC basis alone. The set of configurations included in the LC is selected with an iterative procedure as follows. The LC initially comprises only a small number of the configurations with the lowest energies. Taking an assigned set of target CI states as a reference, a second-order perturbative criterion is used to assess which other configurations would improve the results the most, as well as which configurations already in the LC can be eliminated. The Hamiltonian is diagonalized in the new LC set and the procedure is repeated until the marginal improvement of the energy of the target states is below a preestablished threshold.

\section{RESULTS FOR THE BORON ATOM}

In this section we illustrate the capabilities of the procedure described in Sec. II by using it to compute bound and autoionizing states of the neutral boron atom with ${ }^{2} S^{e}$, ${ }^{2} P^{e, o}$, and ${ }^{2} D^{e}$ symmetry, oscillator strengths between bound states, as well as photoionization cross sections from the $2 s^{2} 2 p$ ground state to selected energy regions of the even manifolds. Whenever possible, the present theoretical results are compared with experimental and theoretical data from the literature.

\section{A. Target B II states}

The B II parent-ion states needed to define the CC expansion for the neutral boron atom are obtained by diagonalizing the two-active-electron effective Hamiltonian of the ion [see Eq. (6)] on the full-CI basis generated from a set of localized orbitals. In the present case, a maximum orbital angular momentum $\ell=6$ is employed, whereas the orbital radial component is expressed in terms of a linear combination of $B$ splines of order 8 [104], defined by a nonuniform grid of 30 nodes, spanning a radial range of $120 \mathrm{Bohr}$ radii. The $\mathrm{CI}$ space comprises a few thousands configurations for each symmetry. The energy for the $2 s^{2}$ ground state calculated in this basis is -24.304540 a.u., which exceeds the experimental value of -24.353148 a.u. [108] by 0.048608 a.u. This difference is in good agreement with the theoretically estimated value for the $K$-shell correlation energy in the neutral, $\Delta E_{c}^{\prime}(2,5)=0.044735$ a.u. (compare with Eq. 12 in [109]). While not included in the present calculations, core correlation is not expected to significantly influence the energy difference between valence-excited states. Table I reports the absolute energy, in a.u., of the first 20 parent-ion states computed in this work, alongside their relative energies with 
TABLE I. Energies of B II bound states.

\begin{tabular}{|c|c|c|c|c|c|}
\hline \multirow[b]{2}{*}{ Configuration } & \multirow[b]{2}{*}{ Symmetry } & \multirow[b]{2}{*}{ This work (a.u.) } & \multicolumn{2}{|c|}{$E-E_{2 s^{2}}(\mathrm{eV})$} & \multirow{2}{*}{$\begin{array}{c}\Delta E \\
(\mathrm{meV})\end{array}$} \\
\hline & & & This work & Exp. $(J)[74]$ & \\
\hline $2 s^{2}$ & ${ }^{1} S$ & -24.3045403 & 0.00000 & 0.00000 & 0 \\
\hline \multirow[t]{2}{*}{$2 s 2 p$} & ${ }^{3} P$ & -24.1340457 & 4.63916 & $4.629017(0)$ & 10 \\
\hline & & & & $4.631777(2)$ & 7 \\
\hline $2 s 2 p$ & ${ }^{1} P$ & -23.9694165 & 9.11872 & 9.1000074 & 19 \\
\hline \multirow[t]{2}{*}{$2 p^{2}$} & ${ }^{3} P$ & -23.8529647 & 12.28737 & $12.263448(0)$ & 24 \\
\hline & & & & $12.266161(2)$ & 21 \\
\hline $2 p^{2}$ & ${ }^{1} D$ & -23.8375559 & 12.70665 & 12.6913659 & 15 \\
\hline $2 p^{2}$ & ${ }^{1} S$ & -23.7225600 & 15.83569 & 15.827970 & 8 \\
\hline $2 s 3 s$ & ${ }^{3} S$ & -23.7130298 & 16.09500 & 16.089904 & 5 \\
\hline $2 s 3 s$ & ${ }^{1} S$ & -23.6772255 & 17.06924 & 17.062984 & 6 \\
\hline \multirow[t]{2}{*}{$2 s 3 p$} & ${ }^{3} P$ & -23.6482411 & 17.85790 & $17.852478(0)$ & 5 \\
\hline & & & & $17.852994(2)$ & 5 \\
\hline $2 s 3 p$ & ${ }^{1} P$ & -23.6476403 & 17.87425 & 17.866487 & 8 \\
\hline $2 s 3 d$ & ${ }^{3} D$ & -23.6179836 & 18.68121 & 18.678180 & 3 \\
\hline $2 s 3 d$ & ${ }^{1} D$ & -23.5995313 & 19.18330 & 19.178634 & 5 \\
\hline $2 s 4 s$ & ${ }^{3} S$ & -23.5464303 & 20.62818 & 20.624138 & 4 \\
\hline $2 s 4 s$ & ${ }^{1} S$ & -23.5392220 & 20.82431 & 20.821325 & 3 \\
\hline $2 s 4 p$ & ${ }^{1} P$ & -23.5270406 & 21.15577 & 21.150629 & 5 \\
\hline $2 s 4 p$ & ${ }^{3} P$ & -23.5227275 & 21.27313 & 21.268988 & 4 \\
\hline $2 s 4 d$ & ${ }^{3} D$ & -23.5112449 & 21.58557 & 21.582342 & 3 \\
\hline $2 s 4 f$ & ${ }^{3} F$ & -23.5074603 & 21.68855 & 21.685249 & 3 \\
\hline $2 s 4 f$ & ${ }^{1} F$ & -23.5073747 & 21.69088 & 21.687550 & 3 \\
\hline $2 s 4 d$ & ${ }^{1} D$ & -23.5045055 & 21.76895 & 21.765055 & 4 \\
\hline
\end{tabular}

respect to the $2 s^{2}$ ground state in electron volts, computed as

$$
\bar{E}_{i(\text { bound })}=R_{\bar{B}}\left(E_{i}^{\mathrm{th}}-E_{2 s^{2}}^{\mathrm{th}}\right),
$$

where the factor $R_{\bar{B}}=\frac{\mu_{\bar{B}}}{m_{e}} R_{\infty}=27.210004 \mathrm{eV}$ takes into account the reduced mass of the $\mathrm{B}^{2+}-e^{-}$system, using for the $\mathrm{B}^{2+}$ ion an average of its natural isotopes $\left(R_{\infty}=\right.$ $\left.27.211385 \mathrm{eV}, \mu_{\bar{B}} / m_{e}=0.99994926\right)$. In the same table, the latter theoretical values are also compared with the corresponding experimental quantities taken from the literature, with which they are in excellent agreement. For the first five excited states, which have either $2 s 2 p$ or $2 p^{2}$ dominant configuration, the discrepancy between the theoretical and the experimental energy is comprised between 7 and $24 \mathrm{meV}$. For the more excited states, however, which have all a single dominant configuration of the form $2 s 3 \ell$ or $2 s 4 \ell$, the agreement is significantly better. To ascertain the convergence of the parention states, we have repeated the CI calculation with two other basis. In one case, the orbital angular momentum was reduced to $\ell=5$, which leads to results differing from those reported in Table I by at most $3 \mathrm{meV}$, but more often by only a fraction of this value. In the other case, nine additional nodes were added at small radii to improve the radial representation of the wave function. The energies obtained in this second case did not differ from those in Table I, within the reported precision.

\section{B. Close-coupling space}

In the present calculation, the nodes defining the diffuse $B$ splines used for Rydberg satellites and asymptotically free electrons have a maximum uniform spacing of 1 a.u., up to a total distance from the nucleus of approximately 300 a.u., where interchannel coupling is assumed to be negligible. Indeed, selected calculations in larger grids show that, in the energy region of interest here, the results are converged with respect to this parameter. After this point, the spacing between consecutive nodes increases rapidly to accommodate the long-range tails of Rydberg states.

In the present work we examine the autoionizing states with either ${ }^{2} P^{o}$ symmetry, i.e., the same symmetry as the $2 s^{2} 2 p$ ground state of the neutral atom, or a symmetry that can be reached from the ground state by means of the absorption of a single photon, within the dipole approximation, namely, the ${ }^{2} S^{e},{ }^{2} P^{e}$, and ${ }^{2} D^{e}$ manifolds. In particular, we focus our attention on the energy region up to the $2 p^{2}\left({ }^{1} S\right)$ threshold for the natural symmetries and up to the next closest threshold, $2 s 3 p\left({ }^{3} P\right)$, in the case of the ${ }^{2} P^{e}$ symmetry. In order to represent correctly the continuum degeneracy and the resonant structure, the CC space must comprise at the very least all the PWCs that open, or whose bound satellites can fall, in this energy region. The essential PWCs for the symmetry and energy region of interest are schematically represented in Fig. 1, alongside the discrete component of each PWC spectrum. This representation already gives an indicative idea of the resonant structure of the ionization continuum. Single-channel bound states located above the first ionization threshold [with the exception of the $2 s 2 p^{2}\left({ }^{1} \mathrm{~S}\right)$ state; see discussion below] give rise to autoionizing resonances. States falling below the threshold that precedes the limit of the series they belong to give rise to intruder states and are tagged with an asterisk. The figure also singles out redundant states, i.e., those bound states of a PWC that are already approximately represented by a bound state that belongs to a channel with a 


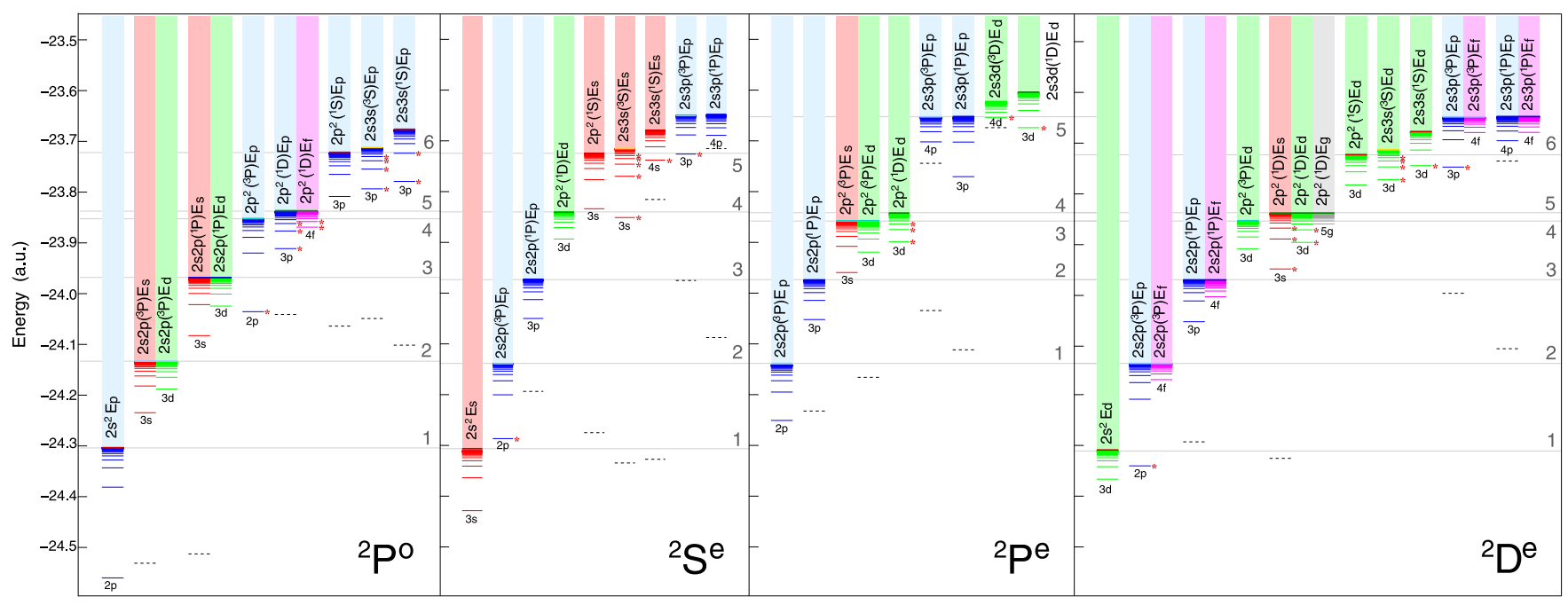

FIG. 1. Energy-level schemes of the single-ionization continuum of the boron atom for the four doublet symmetries examined in this work. The figure shows only the set of channels that are essential to the representation of the autoionizing states of up to the $2 p^{2}\left({ }^{1} S\right)$ threshold for the natural symmetries and up to the $2 s 3 p\left({ }^{3} P\right)$ threshold for the ${ }^{2} P^{e}$ symmetry. The actual CC calculation normally comprises other virtual PWCs, which do not qualitatively alter the resonant structure in the energy region of interest, but which improve the description of the parent-ion polarization induced by the outer electron. Each PWC is represented with a vertical stripe starting at the corresponding threshold, representing the PWC ionization continuum, and with the PWC Rydberg series below threshold. Each parent ion is identified by its dominant configuration and symmetry. The PWC states are colored according to the orbital angular momentum $\ell$ of the satellite or free electron: red, blue, green and magenta for $s, p, d$, and $f$ waves, respectively. Horizontal dashed lines identify redundant states that should not be considered when interpreting the spectrum. For example, in ${ }^{2} P^{o}$ symmetry the first bound states in the $2 s^{2} \varepsilon_{p}$ and $2 s 2 p\left({ }^{3} P\right) \varepsilon_{s}$ channels both give an approximation to the $2 s^{2} 2 p$ ground state. When the PWC channels are allowed to interact, the PWC Rydberg series converging to the lowest threshold gives rise to the bound states of each symmetry, whereas the Rydberg series converging to a limit above the first ionization threshold gives rise to autoionizing states. The terms that fall below the threshold preceding the limit of the series they belong to give rise to intruder states and have been indicated with an asterisk. In the case of the $2 s 2 p^{2}\left({ }^{1} S\right)$ state, the first of the $2 s 2 p\left({ }^{3} P\right) n p\left({ }^{1} S\right)$ series, the energy of the PWC state is actually above the first ionization threshold. However, the double occupancy of the $2 p$ orbital in the main configuration of the first term in the series causes a substantial relaxation of the $2 p$ orbital, if compared to the $2 s 2 p\left({ }^{3} P\right)$ parent ion, which results in the energy of the term to drop below threshold, thus giving rise to an intruder in the $2 s^{2} n s$ bound-state series.

lower threshold. For example, in the case of the ${ }^{2} P^{o}$ symmetry, the ground states of both the $2 s 2 p\left({ }^{3} P\right) \varepsilon_{s}$ and the $2 s 2 p\left({ }^{1} P\right) \varepsilon_{s}$ channels are just slightly different approximations of the $2 s^{2} 2 p$ ground state of the atom, already represented by the first bound state of the $2 s^{2}\left({ }^{1} S\right) \varepsilon_{p}$ channel. When estimating the location of bound and resonant states, all such redundant states must be ignored. In the CC expansion, beyond the essential PWCs shown in Fig. 1, we also include a few additional virtual channels that improve the description of the parent-ion polarization induced by an electron at shortand mid-range distances: the $2 s 3 d\left({ }^{3,1} D\right) \varepsilon_{d}, 2 s 4 s\left({ }^{3,1} S\right) \varepsilon_{s}$, and $2 s 4 p\left({ }^{1,3} P\right) \varepsilon_{p}$ channels in ${ }^{2} \mathrm{~S}^{e}$ symmetry; the $2 s 3 p\left({ }^{1,3} P\right) \varepsilon_{s, d}$ channels in ${ }^{2} P^{o}$ symmetry; the $2 s 4 p\left({ }^{1,3} P\right) \varepsilon_{p}, 2 s 4 d\left({ }^{1,3} D\right) \varepsilon_{d}$, and $2 s 4 f\left({ }^{1,3} F\right) \varepsilon_{f}$ channels in ${ }^{2} P^{e}$ symmetry; and, finally, the $2 s 3 d\left({ }^{3} D\right) \varepsilon_{s, d, g}$ channels in ${ }^{2} D^{e}$ symmetry. The LC, selected with the iterative procedure described in Sec. II from the full-CI space generated by localized orbitals with maximum orbital angular momentum $\ell=6$, counts 5706, 4376, 8582, and 5413 configurations for the ${ }^{2} S^{e},{ }^{2} P^{o},{ }^{2} P^{e}$, and ${ }^{2} D^{e}$ symmetry, respectively. In all the tests we conducted, further expansion of the LC basis to sizes larger than the original selected value by a significant fraction yielded improvements of only few $\mathrm{cm}^{-1}$ for the final energies and equally negligible changes in other quantities such as polarizability, transition probabilities, resonance positions, and widths.

\section{Bound states of B I}

The literature reports several theoretical results for the bound states of B I and related properties calculated by various techniques [49,55-59,109-115] and with an accuracy that, in some cases, is very high. A comprehensive set of the experimental bound-state properties of the neutral boron atom are collected, alongside the most reliable theoretical results, in the review by Fuhr and Wiese [116], which comprises results published across several years. Here we report extensive bound-state properties computed with the method illustrated above to check their accuracy in comparison with published experimental and theoretical benchmarks, as well as to expand the existing database.

The bound states for the ${ }^{2} P^{o},{ }^{2} S^{e},{ }^{2} D^{e},{ }^{2} P^{e}$ manifolds are obtained by diagonalizing the Hamiltonian on the same basis used to compute the continuum states. The results are listed in Table II, together with their corresponding term energies, defined as the energy relative to the $2 s^{2}$ ionization threshold. In this case, the term energy is expressed in wave numbers, using the conversion formula (9), with $1 \mathrm{eV}=8065.544 \mathrm{~cm}^{-1}$, and compared with the weighted average of the available multiplet experimental values. Additional values are provided in the Supplemental Material [117]. Similarly to what was observed in the case of the parent ion, the present ground-state energy for the ${ }^{2} P^{o}$ symmetry differs from the most accurate theoretical 
TABLE II. Energies of the first ${ }^{2} P^{o},{ }^{2} S^{e},{ }^{2} D^{e}$, and ${ }^{2} P^{e}$ bound states of the neutral boron atom. Term energies, $T=E_{2 s^{2}}-E$, are expressed in $\mathrm{cm}^{-1}$. The experimental term energies, which are reported in the second row of each entry and are taken from [108], have been rounded to the first decimal digit. The experimental energies of ten additional ${ }^{2} D^{e}$ states that are also available in the literature differ from the theoretical data obtained in this work by $0.2 \mathrm{~cm}^{-1}$ or less.

\begin{tabular}{|c|c|c|c|c|c|c|c|c|c|c|c|}
\hline \multicolumn{3}{|c|}{${ }^{2} P^{o}$} & \multicolumn{3}{|c|}{${ }^{2} S^{e}$} & \multicolumn{3}{|c|}{${ }^{2} P^{e}$} & \multicolumn{3}{|c|}{${ }^{2} D^{e}$} \\
\hline$E^{\text {th }}$ (a.u.) & $n^{*}$ & $T$ & $E^{\text {th }}$ (a.u.) & $n^{*}$ & $T$ & $E^{\text {th }}$ (a.u.) & $n^{*}$ & $T$ & $E^{\text {th }}$ (a.u.) & $n^{*}$ & $T$ \\
\hline-24.6092411 & 1.281 & $\begin{array}{l}-66877.7 \\
-66928.4\end{array}$ & -24 & 2.020 & $\begin{array}{l}-26886.2 \\
-26888.4\end{array}$ & -24.2782652 & 1.862 & $\begin{array}{l}-31650.9 \\
-31768.8\end{array}$ & -24.39098575 & 2.405 & $\begin{array}{l}-18971.6 \\
-19071.0\end{array}$ \\
\hline-24.3879622 & 2.448 & $\begin{array}{l}-18308.1 \\
-18315.0\end{array}$ & -24.3588371 & 3.035 & $\begin{array}{l}-11916.2 \\
-11917.8\end{array}$ & -24.2131735 & 2.514 & $\begin{array}{l}-17365.7 \\
-17436.4\end{array}$ & -24.35989854 & 3.005 & $\begin{array}{l}-12149.1 \\
-12160.2\end{array}$ \\
\hline-24.3461810 & 3.465 & $\begin{array}{l}-9138.6 \\
-9141.2\end{array}$ & -24.3354263 & 4.024 & $\begin{array}{l}-6778.4 \\
-6781.6\end{array}$ & -24.1758605 & 3.458 & $\begin{array}{l}-9176.8 \\
-8849.4\end{array}$ & -24.33612596 & 3.979 & $\begin{array}{l}-6931.9 \\
-6935.0\end{array}$ \\
\hline-24.3295651 & 4.470 & $\begin{array}{l}-5492.0 \\
-5494.5\end{array}$ & -24.3247445 & 4.975 & $\begin{array}{l}-4434.1 \\
-4445.9\end{array}$ & -24.1593297 & 4.447 & $\begin{array}{l}-5548.9 \\
-5377.9\end{array}$ & -24.32477710 & 4.971 & $\begin{array}{l}-4441.2 \\
-4442.5\end{array}$ \\
\hline-24.3212405 & 5.472 & $\begin{array}{l}-3665.1 \\
-3664.8\end{array}$ & -24.3196524 & 5.752 & $\begin{array}{l}-3316.6 \\
-3367.4\end{array}$ & -24.1509227 & 5.443 & $\begin{array}{l}-3703.9 \\
-3618.5\end{array}$ & -24.31858401 & 5.967 & $\begin{array}{l}-3082.1 \\
-3082.7\end{array}$ \\
\hline-24.3164752 & 6.473 & $\begin{array}{l}-2619.3 \\
-2619.7\end{array}$ & -24.3170193 & 6.330 & $\begin{array}{l}-2738.7 \\
-2772.0\end{array}$ & -24.1460975 & 6.441 & -2644.9 & -24.31484818 & 6.965 & $\begin{array}{l}-2262.2 \\
-2262.7\end{array}$ \\
\hline-24.3134936 & 7.473 & $\begin{array}{l}-1964.9 \\
-1963.7\end{array}$ & -24.3142393 & 7.180 & $\begin{array}{l}-2128.6 \\
-2136.0\end{array}$ & -24.1430785 & 7.440 & -1982.4 & -24.31242497 & 7.964 & $\begin{array}{l}-1730.4 \\
-1730.8\end{array}$ \\
\hline-24.3115045 & 8.473 & -1528.4 & -24.3120817 & 8.143 & $\begin{array}{l}-1655.1 \\
-1657.9\end{array}$ & -24.1410660 & 8.440 & -1540.7 & -24.31076507 & 8.963 & $\begin{array}{l}-1366.1 \\
-1366.4\end{array}$ \\
\hline-24.3101116 & 9.474 & -1222.7 & -24.3105416 & 9.128 & $\begin{array}{l}-1317.1 \\
-1318.7\end{array}$ & -24.1396579 & 9.439 & -1231.7 & -24.30957879 & 9.962 & $\begin{array}{l}-1105.8 \\
-1105.9\end{array}$ \\
\hline-24.3090984 & 10.474 & -1000.3 & -24.3094224 & 10.120 & -1071.4 & -24.1386344 & 10.439 & -1007.0 & -24.30870180 & 10.962 & $\begin{array}{l}-913.3 \\
-913.5\end{array}$ \\
\hline
\end{tabular}

nonrelativistic result reported in the literature, -24.65393 a.u. [115], by 0.04465 a.u., which is in excellent agreement

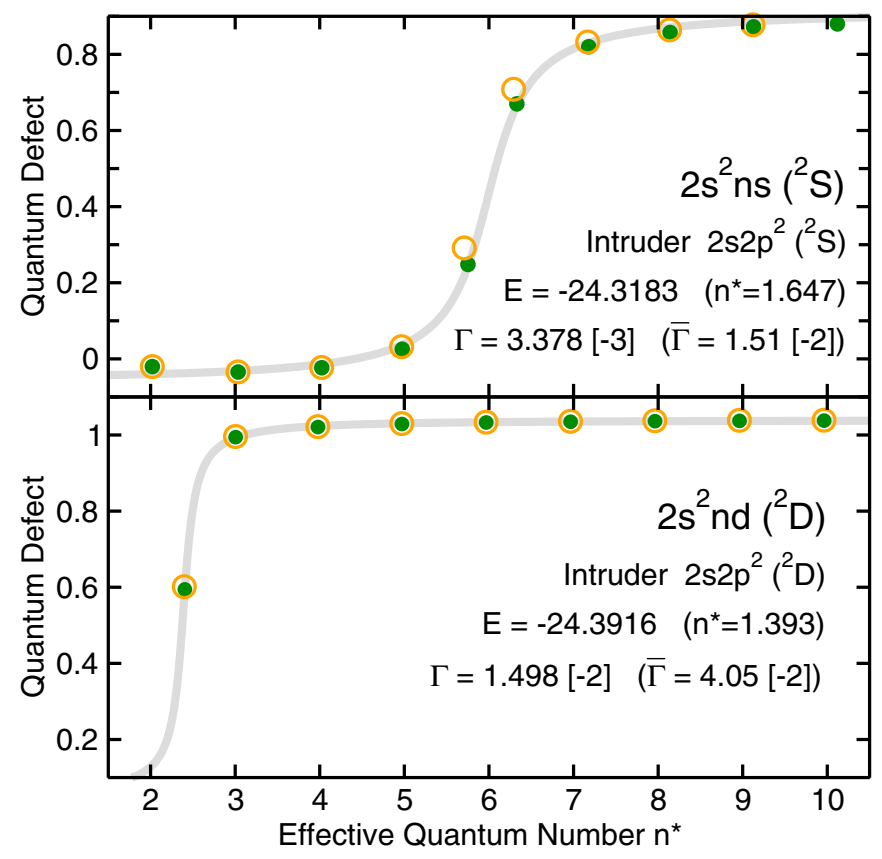

FIG. 2. Quantum defect $\mu$ of the bound ${ }^{2} S^{e}$ states (top) and ${ }^{2} D^{e}$ states (bottom) converging to the $2 s^{2}$ threshold, as a function of the effective quantum number $n^{*}$ (green solid dots). Both series feature an intruder state with $2 s 2 p^{2}$ character. Experimental data are shown as open orange circles. Energies $(E)$, widths $(\Gamma)$, and reduced widths $(\bar{\Gamma})$ are all expressed in atomic units. with the estimated $K$-shell correlation energy $\Delta E_{c}^{\prime}(2,5)=$ 0.044735 a.u. [109]. Regarding the comparison with the experimental term energies, in most cases the agreement is within a few $\mathrm{cm}^{-1}$. Only in a couple of instances does the separation between theoretical and experimental value exceed $100 \mathrm{~cm}^{-1}$. As expected, the agreement between experimental and theoretical term energies is better for the most excited states, since in that case one of the three electrons is mainly localized out of the $L$ shell. In particular, the calculated energies correctly reproduce the ordering observed in the experiment, with the sole exception of the almost degenerate $2 s^{2} 6 s$ and $2 s^{2} 5 d$ states.

A closer look at the quantum defect $\mu_{n} \equiv n-n_{n}^{*}$ of the ${ }^{2} S^{e}$ and ${ }^{2} D^{e}$ bound states, where $n_{n}^{*}$ is the effective principal quantum number with respect to the first ionization threshold, $n_{n}^{*}=\left[2\left(E_{2 s^{2}}-E_{n}\right)\right]^{-1 / 2}$, reveals that in both cases $\mu_{n}$ deviates from the approximately constant value that would be expected for a series of states well approximated by a regular sequence of $2 s^{2} n s$ or $2 s^{2} n d$ configurations (see Fig. 2). This phenomenon is due to the strong mixing of these main-series configurations with the $2 s 2 p^{2}\left({ }^{1} S\right)$ and $2 s 2 p^{2}\left({ }^{1} D\right)$ configurations, respectively, which are approximated by the first bound term of the $2 s 2 p\left({ }^{3} P\right) \varepsilon_{p}$ channels. Such mixing can be construed as the first examples of an intruder state within a Rydberg series (see, e.g., Sec. 3.2.4 in [118]). In ${ }^{2} S^{e}$ symmetry, the first bound state of the $2 s 2 p\left({ }^{1} P\right) \varepsilon_{p}$ PWC is located above the $1 s^{2} \varepsilon_{s}$ threshold. In principle, therefore, this state could appear as the first term of the $2 s 2 p\left({ }^{3} P\right) n_{p}$ autoionizing series. As a result of the interchannel coupling, however, the energy of this state drops below the ionization threshold, where it gives rise to an intruder of the $2 s^{2} n_{s}$ 
TABLE III. Oscillator strength of selected ${ }^{2} P^{o} \rightarrow{ }^{2} S^{e},{ }^{2} D^{e},{ }^{2} P^{e}$ bound-bound transitions. The notation 7.87[-2](3.29) stands for 7.87 $\times$ $10^{-2}$ in length gauge, whereas the value in velocity gauge is $3.29 \%$ larger.

\begin{tabular}{|c|c|c|c|}
\hline & \multicolumn{3}{|c|}{${ }^{2} P^{o} \rightarrow{ }^{2} S^{e}$} \\
\hline & $2 s^{2} 2 p$ & $2 s^{2} 3 p$ & $2 s^{2} 4 p$ \\
\hline $2 s^{2} 3 s$ & $\begin{array}{l}7.87[1-2](-3.29) \\
7.83[-2]^{\mathrm{a}}\end{array}$ & $\begin{array}{l}-1.05(-0.87) \\
-1.05^{\mathrm{b}}\end{array}$ & $\begin{array}{l}-2.67[-3](-5.51) \\
-3.21[-3]^{\mathrm{b}}\end{array}$ \\
\hline $2 s^{2} 4 s$ & $\begin{array}{l}1.63[-2](-3.88) \\
1.54[-2]^{\mathrm{b}}\end{array}$ & $\begin{array}{l}2.02[-1](-0.76) \\
2.03[-1]^{\mathrm{b}}\end{array}$ & \\
\hline $2 s^{2} 5 s$ & $\begin{array}{l}1.06[-2](-3.80) \\
8.20[-3]^{\mathrm{b}}\end{array}$ & $\begin{array}{l}2.05[-2](-0.89) \\
2.07[-2]^{\mathrm{b}}\end{array}$ & $\begin{array}{l}3.33[-1](-0.25) \\
3.35[-1]^{\mathrm{b}}\end{array}$ \\
\hline $2 s^{2} 6 s$ & $\begin{array}{l}1.48[-2](-3.61) \\
1.08[-2]^{\mathrm{b}}\end{array}$ & $\begin{array}{l}6.14[-3](-0.05) \\
6.71[-3]^{\mathrm{c}}\end{array}$ & $\begin{array}{l}2.44[-2](-0.14) \\
2.77[-3]^{\mathrm{c}}\end{array}$ \\
\hline $2 s^{2} 7 s$ & $\begin{array}{l}3.53[-2](-3.34) \\
1.64[-2]^{\mathrm{b}}\end{array}$ & $\begin{array}{l}6.78[-4](-7.19) \\
3.22[-3]^{\mathrm{b}}\end{array}$ & $\begin{array}{l}5.32[-4](-8.87) \\
8.98[-3]^{\mathrm{c}}\end{array}$ \\
\hline $2 s^{2} 8 s$ & $\begin{array}{l}1.78[-2](-3.10) \\
7.4[-3]^{\mathrm{b}}\end{array}$ & $\begin{array}{l}9.57[-4](-8.47) \\
1.57[-3]^{\mathrm{b}}\end{array}$ & $\begin{array}{l}5.36[-3](-3.60) \\
3.03[-3]^{\mathrm{c}}\end{array}$ \\
\hline $2 s^{2} 9 s$ & $\begin{array}{l}2.38[-3](-2.70) \\
1 .[-2]^{\mathrm{b}}\end{array}$ & $\begin{array}{c}1.48[-3](-4.29) \\
{ }^{2} P^{o} \rightarrow{ }^{2} P^{e}\end{array}$ & $5.03[-3](-2.24)$ \\
\hline $2 s 2 p^{2}$ & \begin{tabular}{l}
\multicolumn{1}{c}{$2 s^{2} 2 p$} \\
$5.98[-1](4.41)$ \\
$5.85[-1]^{c}$
\end{tabular} & $\begin{array}{l}2 s^{2} 3 p \\
1.51[-3](-0.48) \\
1.6[-3]^{c}\end{array}$ & \begin{tabular}{l}
\multicolumn{1}{c}{$2 s^{2} 4 p$} \\
$5.18[-4](0.19)$ \\
$6.6[-4]^{c}$
\end{tabular} \\
\hline $2 s 2 p\left({ }^{3} P\right) 3 p$ & $\begin{array}{l}4.06[-2](10.22) \\
5.8[-2]^{\mathrm{c}}\end{array}$ & $\begin{array}{l}3.96[-3](2.70) \\
3.5[-3]^{\mathrm{c}}\end{array}$ & $\begin{array}{l}1.68[-3](1.79) \\
1.52[-3]^{\mathrm{c}}\end{array}$ \\
\hline $2 s 2 p\left({ }^{3} P\right) 4 p$ & $\begin{array}{l}1.70[-3](22.61) \\
3.5[-3]^{\mathrm{c}}\end{array}$ & $\begin{array}{l}1.70[-3](3.46) \\
1.7[-3]^{\mathrm{c}}\end{array}$ & $\begin{array}{l}4.86[-4](2.94) \\
5.1[-4]^{\mathrm{c}}\end{array}$ \\
\hline $2 s 2 p\left({ }^{3} P\right) 5 p$ & $1.69[-4](47.23)$ & $\begin{array}{c}8.11[-4](3.38) \\
{ }^{2} P^{o} \rightarrow{ }^{2} D^{e}\end{array}$ & $1.98[-4](2.65)$ \\
\hline $2 s 2 p^{2}$ & $\begin{array}{l}\quad 2 s^{2} 2 p \\
4.58[-2](12.24) \\
4.71[-2]^{\mathrm{a}}\end{array}$ & $2 s^{2} 3 p$ & $2 s^{2} 4 p$ \\
\hline $2 s^{2} 3 d$ & $\begin{array}{l}1.70[-1](1.48) \\
1.70[-1]^{\mathrm{a}}\end{array}$ & $\begin{array}{l}8.32[-1](0.08) \\
8.35[-1]^{\mathrm{a}}\end{array}$ & \\
\hline $2 s^{2} 4 d$ & $\begin{array}{l}7.02[-2](0.84) \\
7.22[-2]^{b}\end{array}$ & $\begin{array}{l}9.93[-6](14.82) \\
6.6[-6]^{b}\end{array}$ & $\begin{array}{l}1.26(0.18) \\
1.26^{\mathrm{b}}\end{array}$ \\
\hline $2 s^{2} 5 d$ & $\begin{array}{l}3.48[-2](0.68) \\
3.58[-2]^{\mathrm{b}}\end{array}$ & $\begin{array}{l}2.84[-3](0.72) \\
2.57[-3]^{\mathrm{b}}\end{array}$ & $\begin{array}{l}9.78[-3](0.37) \\
1.08[-2]^{\mathrm{b}}\end{array}$ \\
\hline $2 s^{2} 6 d$ & $\begin{array}{l}1.97[-2](0.75) \\
2.06[-2]^{\mathrm{c}}\end{array}$ & $\begin{array}{l}2.74[-3](0.97) \\
2.60[-3]^{\mathrm{c}}\end{array}$ & $\begin{array}{l}1.08[-4](-0.48) \\
1.72[-4]^{\mathrm{c}}\end{array}$ \\
\hline $2 s^{2} 7 d$ & $\begin{array}{l}1.23[-2](0.75) \\
1.27[-2]^{\mathrm{c}}\end{array}$ & $\begin{array}{l}2.09[-3](0.94) \\
2.01[-3]^{\mathrm{c}}\end{array}$ & $7.70[-5](1.04)$ \\
\hline $2 s^{2} 8 d$ & $\begin{array}{l}8.14[-3](0.73) \\
8.46[-3]^{c}\end{array}$ & $\begin{array}{l}1.54[-3](0.91) \\
1.49[-3]^{\mathrm{c}}\end{array}$ & $\begin{array}{l}1.87[-4](0.66) \\
1.57[-4]^{c}\end{array}$ \\
\hline
\end{tabular}

${ }^{\mathrm{a}}$ Tachiev and Froese-Fischer [49].

${ }^{\mathrm{b}}$ Fernley et al. [112].

${ }^{\mathrm{c}}$ Opacity Project [32].

series, located around $n_{s}^{*}=6.02$. The interchannel interaction is strong enough to mix the state with several terms of the $2 s^{2} n_{s}$ Rydberg series. From the $\mu_{n}$ values, it is possible to extrapolate a position $E_{2 s 2 p^{2}\left({ }^{2} S\right)}$ and a width $\Gamma_{2 s 2 p^{2}\left({ }^{2} S\right)}$ for the intruder state as if it were a resonance interacting with the discretized continuum realized by the $2 s^{2} n s$ Rydberg series. This is done by fitting the quantum defect with the function $\mu(E)=$ $c_{0}+1 / 2+\pi^{-1} \arctan \left[2\left(E-E_{2 s 2 p^{2}\left({ }^{2} S\right)}\right) / \Gamma_{2 s 2 p^{2}\left({ }^{2} S\right)}\right]$, where $c_{0}, E_{2 s 2 p^{2}\left({ }^{2} S\right)}$, and $\Gamma_{2 s 2 p^{2}\left({ }^{2} S\right)}$ are free parameters. The energy and width estimated in this way are $E_{2 s 2 p^{2}\left({ }^{2} S\right)} \simeq-24.3183$ a.u. and $\Gamma_{2 s 2 p^{2}\left({ }^{2} S\right)}=0.0034(3)$ a.u., which correspond to an effective quantum defect with respect to the $2 s 2 p\left({ }^{3} P\right)$ threshold $n_{2 s 2 p^{2}\left({ }^{2} S\right)}^{*}=1.647$ and to a reduced width $\bar{\Gamma}_{2 s 2 p^{2}\left({ }^{2} S\right)}=$ $\Gamma_{2 s 2 p^{2}\left({ }^{2} S\right)}\left(n_{2 s 2 p^{2}\left({ }^{2} S\right)}^{*}\right)^{3}=0.015$ a.u. The effective quantum defect for this state, $\mu=2-1.647=0.353$, is reasonably close to the value $\sim 0.267$ observed for the higher terms in the $2 s 2 p\left({ }^{3} P\right) n_{p}$ autoionizing series (compare with Table VIII). A slightly larger value for the quantum defect of the first term in the series is justified since the two outer electrons occupy the 
TABLE IV. Oscillator strength of ${ }^{2} D^{e} \rightarrow{ }^{2} P^{o},{ }^{2} D^{e} \rightarrow{ }^{2}$ $D^{o},{ }^{2} S^{e} \rightarrow{ }^{2} P^{o}$, and ${ }^{2} D^{e} \rightarrow{ }^{2} F^{o}$ bound-bound transitions.

\begin{tabular}{|c|c|c|}
\hline Initial & Final & $f$ \\
\hline $2 s 2 p^{2}$ & $2 s^{2} 3 p$ & $\begin{array}{l}4.26[-3](24.76) \\
4.42[-3]^{\mathrm{a}}\end{array}$ \\
\hline " & $2 s^{2} 4 p$ & $\begin{array}{l}3.00[-5](57.51) \\
3.2[-5]^{\mathrm{a}}\end{array}$ \\
\hline " & $2 s 2 p\left({ }^{3} P\right) 3 d\left({ }^{2} D\right)$ & $\begin{array}{l}4.28[-3](-17.65) \\
5.5[-3]^{\mathrm{a}}\end{array}$ \\
\hline " & $2 s^{2} 4 f$ & $\begin{array}{l}3.49[-2](0.17) \\
4.84[-2]^{\mathrm{b}}\end{array}$ \\
\hline " & $2 s^{2} 5 f$ & $\begin{array}{l}1.50[-2](0.58) \\
2.1[-2]^{\mathrm{a}}\end{array}$ \\
\hline " & $2 s^{2} 6 f$ & $\begin{array}{l}7.82[-3](0.35) \\
1.1[-2]^{\mathrm{a}}\end{array}$ \\
\hline " & $2 s^{2} 7 f$ & $\begin{array}{l}4.63[-3](0.54) \\
6.2[-3]^{\mathrm{a}}\end{array}$ \\
\hline " & $2 s^{2} 8 f$ & $\begin{array}{c}2.98[-3](0.59) \\
4[-3]^{\mathrm{a}}\end{array}$ \\
\hline $2 s^{2} 3 d$ & $2 s 2 p\left({ }^{3} P\right) 3 d\left({ }^{2} D\right)$ & $\begin{array}{l}9.02[-4](-11.82) \\
1.3[-3]^{\mathrm{a}}\end{array}$ \\
\hline " & $2 s^{2} 4 f$ & $\begin{array}{l}9.60[-1](0.23) \\
9.6[-1]^{b}\end{array}$ \\
\hline " & $2 s^{2} 5 f$ & $\begin{array}{l}1.36[-1](0.24) \\
1.3[-1]^{\mathrm{a}}\end{array}$ \\
\hline " & $2 s^{2} 6 f$ & $\begin{array}{l}4.45[-2](0.29) \\
4.18[-2]^{\mathrm{a}}\end{array}$ \\
\hline " & $2 s^{2} 7 f$ & $\begin{array}{l}2.05[-2](0.50) \\
1.90[-2]^{\mathrm{a}}\end{array}$ \\
\hline " & $2 s^{2} 8 f$ & $\begin{array}{l}1.13[-2](0.57) \\
1.03[-2]^{\mathrm{a}}\end{array}$ \\
\hline $2 s^{2} 4 d$ & $2 s 2 p\left({ }^{3} P\right) 3 d\left({ }^{2} D\right)$ & $\begin{array}{l}2.79[-4](-9.60) \\
3.7[-4]^{\mathrm{a}}\end{array}$ \\
\hline " & $2 s^{2} 5 f$ & $\begin{array}{l}8.55[-1](0.21) \\
8.92[-1]^{\mathrm{a}}\end{array}$ \\
\hline " & $2 s^{2} 6 f$ & $\begin{array}{l}1.82[-1](0.32) \\
1.84[-1]^{\mathrm{a}}\end{array}$ \\
\hline " & $2 s^{2} 7 f$ & $\begin{array}{l}7.09[-2](0.50) \\
7.08[-2]^{\mathrm{a}}\end{array}$ \\
\hline " & $2 s^{2} 8 f$ & $\begin{array}{l}3.60[-2](0.57) \\
3.57[-2]^{\mathrm{a}}\end{array}$ \\
\hline $2 s^{2} 5 d$ & $2 s^{2} 6 f$ & $\begin{array}{l}7.88[-1](0.12) \\
8.26[-1]^{\mathrm{a}}\end{array}$ \\
\hline " & $2 s^{2} 7 f$ & $\begin{array}{l}1.91[-1](0.29) \\
1.95[-1]^{\mathrm{a}}\end{array}$ \\
\hline " & $2 s^{2} 8 f$ & $\begin{array}{l}7.97[-2](0.37) \\
8.05[-2]^{\mathrm{a}}\end{array}$ \\
\hline $2 s^{2} 6 d$ & $2 s^{2} 7 f$ & $\begin{array}{c}7.60[-1](0.12) \\
8[-1]^{\mathrm{a}}\end{array}$ \\
\hline " & $2 s^{2} 8 f$ & $\begin{array}{l}1.95[-1](0.21) \\
1.99[-1]^{\mathrm{a}}\end{array}$ \\
\hline $2 s^{2} 7 d$ & $2 s^{2} 8 f$ & $\begin{array}{l}7.52[-1](0.12) \\
7.9[-1]^{\mathrm{a}}\end{array}$ \\
\hline
\end{tabular}

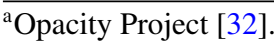

${ }^{\mathrm{b}}$ Fernley et al. [112].

same subshell and their mutual screening is therefore smaller. The reduced width $\bar{\Gamma}_{n}$ of the $2 s 2 p\left({ }^{3} P\right) n_{p}$ resonance series, extrapolated to the energy of the $2 s 2 p^{2}$ intruder state, gives the value $\bar{\Gamma} \simeq 0.0137$ a.u., which, again, is in line with the effective width $\bar{\Gamma}_{2 s 2 p^{2}\left({ }^{2} S\right)}$ found earlier. The good agreement between the present calculations and the experimental values, also shown in Fig. 2, is clearly visible in this representation. In the experimental case, it appears that the ${ }^{2} S$ intruder is centered at a slightly lower energy compared with the calculation. The energy difference is of the order of $6 \mathrm{meV}$, which is perfectly in line with the theoretical overestimation, by $7 \mathrm{meV}$ (for $J=2$ ), of the $E_{2 s 2 p^{3} P}-E_{2 s^{2}}$ gap already seen in Sec. III A (compare with Table I).

Entirely similar considerations can be repeated for ${ }^{2} D^{e} 2 s^{2} n d$ bound states, in relation to the $2 s 2 p^{2}\left({ }^{2} D\right)$ intruder state (see bottom panel in Fig. 2). In this case, the fitting parameters are $E_{2 s 2 p^{2}\left({ }^{2} D\right)}=-24.3916$ a.u. and $\Gamma_{2 s 2 p^{2}\left({ }^{2} D\right)}=$ 0.015 a.u., which correspond to an effective quantum number and a reduced width, with respect to the $2 s 2 p\left({ }^{3} P\right)$ threshold, of $n_{2 s 2 p^{2}\left({ }^{2} D\right)}^{*}=1.393$ and $\bar{\Gamma}_{2 s 2 p^{2}\left({ }^{2} D\right)}=0.0405$ a.u., whereas the values extrapolated from the $2 s 2 p\left({ }^{3} P\right) n p\left({ }^{2} D\right)$ autoionizing series are 1.568 and 0.0451 a.u., respectively (compare with Table XII). Also in this case, the fitted values are in reasonable agreement with the ones extrapolated from above the threshold and in very good agreement with the experimental measurements.

The good agreement between the theoretical and experimental intruderlike features of the ${ }^{2} S^{e}$ and ${ }^{2} D^{e}$ boundstate series is a strong indication that the present $\mathrm{CC}+\mathrm{LC}$ bases represent accurately not only the states associated with the closed-shell $2 s^{2}$ parent ion, but also those associated with the open-shell states as well as their interchannel coupling.

Table III lists the values of the oscillator strength between selected bound states with ${ }^{2} P^{o}$ and with ${ }^{2} S^{e},{ }^{2} P^{e}$, or ${ }^{2} D^{e}$ symmetry and compares them with the values available in the literature. For completeness, in Table IV we also report other transitions for which a comparison with existing literature data is possible: ${ }^{2} D^{e}{ }_{-}^{2} P^{o},{ }^{2} S^{e}{ }_{-}^{2} P^{o},{ }^{2} D^{e}{ }_{-}^{2} D^{o}$ and ${ }^{2} D^{e}{ }_{-}^{2} F^{o}$. So far, we have not discussed the ${ }^{2} D^{o}$ and ${ }^{2} F^{o}$ symmetries, since their resonant structure is beyond the scope of this work. To compute the ${ }^{2} D^{e}{ }_{-}^{2} D^{o}$ and ${ }^{2} D^{e}-{ }^{2} F^{o}$ transitions, the bound states for these two symmetries have also been obtained from a CC + LC space. Oscillator strengths have been computed both in velocity and in length gauge. The present results are compared with the data in Fuhr and Wiese [116], which compile results from multiple sources and with unequal levels of accuracy [49]. The agreement with the available data, especially with the most accurate ones [47], is overall very satisfactory, despite the $\mathrm{CC}+\mathrm{LC}$ approach not being specifically tailored to bound states. For the two transitions $2 s^{2} 2 p\left({ }^{2} P^{o}\right) \rightarrow 2 s 2 p\left({ }^{3} P\right) 4-5 p\left({ }^{2} P^{e}\right)$, the difference between the oscillator strength in length and velocity gauge, $\sim 23 \%$ and $\sim 47 \%$, respectively, is uncharacteristically large. However, it looks like these specific transitions are very sensitive to the accuracy of the states involved, since transitions to the same ${ }^{2} P^{e}$ final states from excited ${ }^{2} P^{o}$ states, or, conversely, from the ${ }^{2} P^{o}$ ground state to bound states in ${ }^{2} S^{e}$ or ${ }^{2} D^{e}$ symmetries, all exhibit a much better gauge agreement. In all the other cases, the gauge agreement is of the order of $10 \%$ and oftentimes much better than that. 


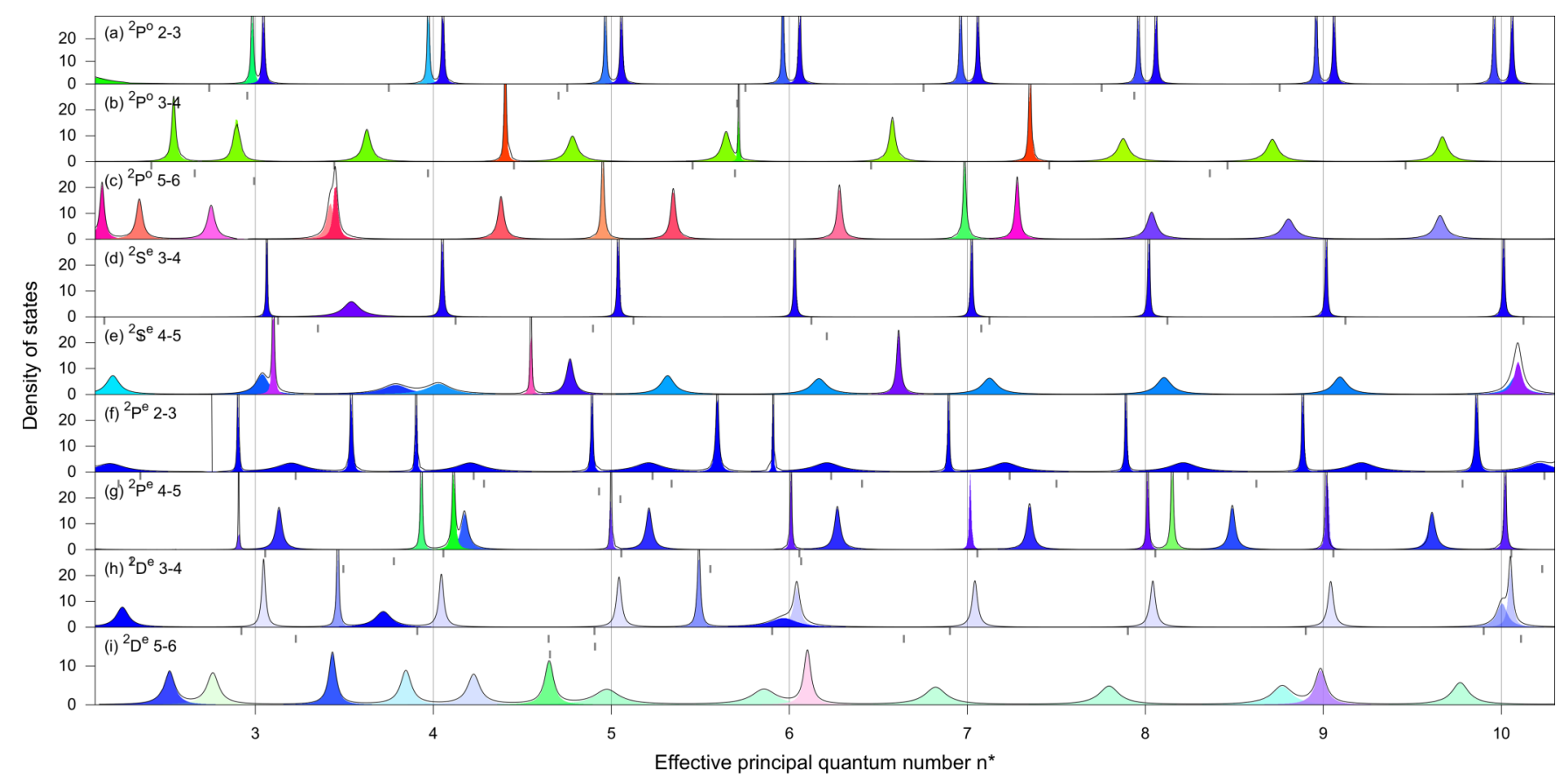

FIG. 3. Density of states $\rho\left(n^{*}\right)$, between selected pairs of consecutive thresholds, $E_{1}<E_{2}$, as a function of the effective principal quantum number $n^{*}=\left[2\left(E_{2}-E\right)\right]^{-1 / 2}$, for the four symmetries considered here: ${ }^{2} P^{o},{ }^{2} S^{e},{ }^{2} P^{e}$, and ${ }^{2} D^{e}$ (for threshold numbering, compare with Fig. 1). The density of states is defined here as $\rho\left(n^{*}\right)=\pi^{-1} \partial_{n^{*}} \varphi_{\text {tot }}$. Each resonance $i$ appears as an almost-Lorentzian peak that integrates to 1 and whose position and width is determined by the effective quantum number $n_{i}$ and by the reduced width $\bar{\Gamma}_{i}$, respectively, $\partial_{n^{*}} \varphi_{i} \simeq \frac{1}{2} \bar{\Gamma}_{i} /\left[4 c_{i}\left(n^{*}-n_{i}\right)^{2}+\left(\bar{\Gamma}_{i} / 2\right)^{2}\right]$, where $\varphi_{i}$ is the contribution of the $i$ th resonance to the total phase shift, while the factor $c_{i}=$ $\left[n^{*} / n_{i}\left(1+n^{*} / n_{i}\right) / 2\right]^{2}$ rapidly approaches 1 as $n^{*} \rightarrow \infty$. Each resonant contribution is separately highlighted with a shaded area, colored with an RGB code in which the red (r), blue (b), and green (g) weights are proportional to the $s, p$, and $d$ character of the electron wave emitted in their decay. A resonance that entirely decays by emitting a $p$ electron, therefore, will appear as blue, one decaying entirely by emitting an $s$ electron will appear as red, one decaying partly in an $s$ and partly in a $p$ wave will appear as magenta, and so on. Finally, the opacity of the shade is proportional to the weight of the $s+p+d$ decay channels with respect to the total (a resonance that decays predominantly emitting $s, p$, or $d$ waves is almost entirely opaque, while one that predominantly emits $f$ or $g$ waves is almost transparent). Vertical gray lines indicate the positions of the Rydberg states, in the single-PWC approximation, that give rise to the autoionizing states. Interchannel coupling often shifts the resonance energy considerably. A better predictor of intruder-state energies, therefore, is obtained by extrapolating the parameters of the corresponding series of autoionizing states from the next energy interval (see text for examples).

\section{Autoionizing states}

In this section we present the results for the autoionizing states within the ${ }^{2} S^{e},{ }^{2} P^{e},{ }^{2} P^{o}$, and ${ }^{2} D^{e}$ manifolds, up to the $2 p^{2}\left({ }^{1} S\right)$ threshold, for the natural symmetries, and up to the $2 s 3 p\left({ }^{3} P\right)$ threshold for the ${ }^{2} P^{e}$ symmetry. As shown in Fig. 1, all four symmetries have rich resonant structures, with both single and multiple Rydberg series decaying to multichannel continua and interspersed by several intruder states. Most of the autoionizing states examined in this work are associated with excited parent ions, whose energy with respect to the $2 s^{2} \mathrm{~B}^{+}$ground state is systematically, if slightly, overestimated compared with the experimental value (compare with Table I). To better predict the location of the resonances above the first ionization threshold, therefore, alongside the absolute energy position in atomic units, we also report their energy above the first ionization threshold, using as a reference the energy of the $2 s 3 s\left({ }^{1} S\right)$ parent ion, which is not split by spin-orbit interaction, it only has a singly occupied $2 s$ orbital, as is the case for most of the autoionizing states, and whose experimental elevation above the $1 s^{2}$ threshold is accurately known, $\bar{E}_{i(\mathrm{res})}=\left[E_{i}^{\mathrm{th}}-\right.$ $\left.E_{2 s 3 s\left({ }^{1} S\right)}^{\text {th }}\right] \mu_{\bar{B}} / m_{e}+E_{2 s 3 s\left({ }^{1} S\right)}^{\exp }-E_{2 s^{2}}^{\exp }$. In numbers, the con- version formula reads $\bar{E}_{i}(\mathrm{eV})=\left[E_{i}^{\text {th }}(\right.$ a.u. $\left.)+23.6772255\right] \times$ $27.210004 \mathrm{eV}+17.062984 \mathrm{eV}$. Figure 3 shows the density of states $\rho\left(n^{*}\right)$, for the four symmetries under examination here, between selected pairs of consecutive thresholds, where each resonance is visible as a quasi-Lorentzian peak. Apart for its position and width, a resonance can also be characterized by its branching ratios, $b_{\alpha, a}=\Gamma_{\alpha, a} / \Gamma_{a}$. To better highlight the differences between resonances, Fig. 3 shows their individual contribution to $\rho\left(n^{*}\right)$ colored according to the proportion with which they decay emitting an $s, p$, or $d$ electron. Regular autoionizing Rydberg series, which are characterized by an almost constant quantum defect $\mu_{i}=i-n_{i}^{*} \simeq \mu_{\infty}$ and reduced partial widths $\bar{\Gamma}_{\alpha, i} \simeq \bar{\Gamma}_{\alpha, \infty}$, appear in Fig. 3 as periodic sequences of identical, equally colored peaks, spaced by $\Delta n=1$. Resonances give rise to asymmetric Fano profiles in the photoionization cross section from a bound state [119], which may also be used to characterize them. In the following, we examine the individual symmetries in each of the energy intervals between consecutive thresholds. In most cases, we assign to the resonances a configuration based on the single-particle approximation. While the given assignment does indicate the dominant configuration, it is to be understood 
TABLE V. Parameters of the ${ }^{2} P^{o}$ resonances between the $2 s^{2}$ and the $2 s 2 p\left({ }^{3} P\right)$ threshold.

\begin{tabular}{lccccc}
\hline \hline State & $E$ (a.u.) & $E(\mathrm{eV})$ & $\Gamma$ (a.u.) & $n^{*}$ & $\bar{\Gamma}$ (a.u.) \\
\hline $2 s 2 p\left({ }^{3} P\right) 3 d$ & -24.189206 & 3.13198 & $5.53[-5]$ & 3.011 & $1.51[-3]$ \\
$2 s 2 p\left({ }^{3} P\right) 4 d$ & -24.165337 & 3.78148 & $2.77[-5]$ & 3.997 & $1.77[-3]$ \\
$2 s 2 p\left({ }^{3} P\right) 5 d$ & -24.154135 & 4.08628 & $1.52[-5]$ & 4.989 & $1.89[-3]$ \\
$2 s 2 p\left({ }^{3} P\right) 6 d$ & -24.148012 & 4.25288 & $9.18[-6]$ & 5.983 & $1.97[-3]$ \\
$2 s 2 p\left({ }^{3} P\right) 7 d$ & -24.144310 & 4.35362 & $5.95[-6]$ & 6.980 & $2.02[-3]$ \\
$2 s 2 p\left({ }^{3} P\right) 8 d$ & -24.141904 & 4.41909 & $4.06[-6]$ & 7.977 & $2.06[-3]$ \\
$2 s 2 p\left({ }^{3} P\right) 9 d$ & -24.140253 & 4.46400 & $2.90[-6]$ & 8.975 & $2.09[-3]$ \\
$2 s 2 p\left({ }^{3} P\right) 10 d$ & -24.139072 & 4.49613 & $2.14[-6]$ & 9.973 & $2.12[-3]$ \\
$2 s 2 p\left({ }^{3} P\right) 3 s$ & -24.243232 & 1.66195 & $5.73[-3]$ & 2.140 & $5.62[-2]$ \\
$2 s 2 p\left({ }^{3} P\right) 4 s$ & -24.184225 & 3.26751 & $1.59[-3]$ & 3.157 & $5.01[-2]$ \\
$2 s 2 p\left({ }^{3} P\right) 5 s$ & -24.162927 & 3.84705 & $6.69[-4]$ & 4.161 & $4.82[-2]$ \\
$2 s 2 p\left({ }^{3} P\right) 6 s$ & -24.152808 & 4.12238 & $3.44[-4]$ & 5.162 & $4.73[-2]$ \\
$2 s 2 p\left({ }^{3} P\right) 7 s$ & -24.147210 & 4.27470 & $2.00[-4]$ & 6.163 & $4.68[-2]$ \\
$2 s 2 p\left({ }^{3} P\right) 8 s$ & -24.143790 & 4.36775 & $1.27[-4]$ & 7.163 & $4.66[-2]$ \\
$2 s 2 p\left({ }^{3} P\right) 9 s$ & -24.141549 & 4.42874 & $8.54[-5]$ & 8.163 & $4.64[-2]$ \\
$2 s 2 p\left({ }^{3} P\right) 10 s$ & -24.140001 & 4.47087 & $6.03[-5]$ & 9.163 & $4.64[-2]$ \\
$2 s 2 p\left({ }^{3} P\right) 11 s$ & -24.138886 & 4.50119 & $4.42[-5]$ & 10.163 & $4.64[-2]$ \\
$2 s 2 p\left({ }^{3} P\right)$ & -24.134046 & 4.63290 & & & \\
\hline \hline
\end{tabular}

that an autoionizing state can occasionally exhibit a significant contamination from other configurations. This is most often the case when an intruder state is close in energy to one or more terms in a main series.

To confirm the parameters of boron autoionizing states computed in this work, it would be useful to have reliable experimental values to compare with. To the best of our knowledge, however, there is only one experimental paper, from 1976, claiming to have determined the position of autoionizing states of boron in symmetries of interest here [120]. In that work, the authors reported a value for the wavelength of the transition between both the $2 s 2 p\left({ }^{3} P\right) 3 p{ }^{2} S$ state and the ${ }^{2} D$ state with the ground $2 s^{2} 2 p$ state. The paper, which also reports measurements for the stronger transitions to the ${ }^{2} P^{e}$ manifold, specifies, on the one hand, that the transition to the $2 s 2 p\left({ }^{3} P\right) 3 p$ states are much weaker, and, on the other hand, provides for both of them spin-orbit-resolved values, with differences between the multiplets that are orders of magnitude smaller than the autoionization width computed here for those resonances. These puzzling circumstances, and the lack of any further details on how the transitions in question were identified or assigned, suggest that new experimental investigations are in order. In any case, in the following the comparison between these two experimental values and the ones computed here is discussed in detail.

\section{1. ${ }^{2} P^{o}$ autoionizing states}

The main field-free parameters of the ${ }^{2} P^{o}$ resonancesposition, width, and branching ratios, up to the sixth threshold - are listed in Tables V, VI, and VII, together with orientative assignments of their dominant configuration (see first panel in Fig. 1). The first energy interval, between the $2 s^{2}$ and the $2 s 2 p\left({ }^{3} P\right)$ threshold, features two main resonance series, which can be identified by comparison with the energy of the bound states in the corresponding PWC. These are the
$2 s 2 p\left({ }^{3} P\right) n s$ series and the narrower $2 s 2 p\left({ }^{3} P\right) n d$ series, both of which are regular; i.e., their quantum defect and reduced partial widths are stable across the series (compare with Table V).

The second energy interval, up to the $2 s 2 p\left({ }^{1} P\right)$ threshold, comprises again two main resonant Rydberg series, decaying to three open channels, and perturbed by one clear intruder, with $2 p^{3}$ dominant configuration. The two $2 s 2 p\left({ }^{1} P\right) n s$ and $2 s 2 p\left({ }^{1} P\right) n d$ series have comparable asymptotic reduced widths, $1.06 \times 10^{-2}$ and $1.01 \cdot 10^{-2}$ a.u., respectively, and they both decay preferentially to the $2 s^{2} \varepsilon_{p}$ channel (see Table VI). In contrast to the main series, the $2 p^{3}$ intruder state has a strong coupling to the $2 s 2 p\left({ }^{3} P\right) \varepsilon_{d}$ channel, which, with a prevalence of almost $90 \%$, completely dominates its branching ratio, and which imparts to the resonance a width more than one order of magnitude larger than that of any other autoionizing state in this interval. The first terms of the two main series, the $2 s 2 p\left({ }^{1} P\right) 3 s$ and $2 s 2 p\left({ }^{1} P\right) 3 d$ states, partly mix with the $2 p^{3}$ state, thus acquiring a noticeably larger decay component to the $\varepsilon_{d}$ channel. It is interesting to notice that the staggering prevalence of the decay of the $2 p^{3}$ state to the $2 s 2 p\left({ }^{3} P\right) \varepsilon_{d}$ channel is not replicated by the higher terms of the $2 p^{2}\left({ }^{3} P\right) n p$ autoionizing series in the next energy region. This circumstance suggests that the $2 p^{3}$ configuration in the intruder state is accompanied to secondary components that account for the large angular correlation between the three degenerated electrons. The density-of-state plot for this energy interval is shown in the top panel in Fig. 3. The upper edge of the $2 p^{3}$ intruder state is visible in green, on the left, owing to its preferential decay through emission of a $d$ electron, while the periodic pairs of narrower peaks represent terms of the two main series. The $2 s 2 p\left({ }^{1} P\right) n s$ series, which is less affected by the interference of the intruder, exhibits a uniform blue color, while the $2 s 2 p\left({ }^{1} P\right) n d$ terms switch gradually from green to blue, due to their partial mixing with the $2 p^{3}$ intruder configuration.

The third energy interval, between the $2 s 2 p\left({ }^{1} P\right)$ and the $2 p^{2}\left({ }^{3} P\right)$ thresholds, harbors at least five intruders from the series converging to the next threshold, $2 p^{2}\left({ }^{1} D\right)$, which is very close in energy to the upper limit of this interval. Four intruder states are clearly visible in the second panel of Fig. 3. Their positions can be estimated by extrapolating their effective principal quantum numbers from those of the terms in the main series from which they supposedly originate. In particular, for the $2 p^{2}\left({ }^{1} D\right) 4 f-5 f$ and $2 p^{2}\left({ }^{1} D\right) 3 p-5 p$, we guess the $n^{*}$ values, relative to the $2 p^{2}\left({ }^{1} D\right), 4.032,5.032,2.580,3.572$, and 4.565 , respectively, which correspond to the apparent $n^{*}$ values, relative to the $2 p^{2}\left({ }^{3} P\right)$ threshold, 5.708, 10.736, $2.894,4.586$, and 7.632, respectively. These values should be compared with those actually observed in the calculation: 5.716, 10.749, 2.895, 4.404, and 7.352. In the first three cases, the position estimated by extrapolating the parameters of the regular series is extremely good. In the last two cases, while the assignment is still qualitatively accurate, a significant deviation is observed. This is because the extrapolated position of the $2 p^{2}\left({ }^{1} D\right) 4 p$ and $2 p^{2}\left({ }^{1} D\right) 5 p$ intruder states, $n_{2 p^{2}\left({ }^{1} D\right) 4 p}^{* \text {, uess }}=4.586$ and $n_{2 p^{2}\left({ }^{1} D\right) 5 p}^{*, \text { uuss }}=7.632$, happen to be very close to the effective quantum numbers, $n_{2 p^{2}\left({ }^{3} P\right) 5 p}^{* \text { Guess }} \simeq 4.64$ and $n_{2 p^{2}\left({ }^{3} P\right) 8 p}^{* \text { Guess }} \simeq 7.64$, 
TABLE VI. Parameters of the ${ }^{2} P^{o}$ resonances between the second, $2 s 2 p\left({ }^{3} P\right)$, and the fifth, $2 p^{2}\left({ }^{1} D\right)$, thresholds. The six channels that open in these first four energy intervals are, in order, $2 s^{2} \varepsilon_{p}, 2 s 2 p\left({ }^{3} P\right) \varepsilon_{s}, 2 s 2 p\left({ }^{3} P\right) \varepsilon_{d}, 2 s 2 p\left({ }^{1} P\right) \varepsilon_{s}, 2 s 2 p\left({ }^{1} P\right) \varepsilon_{d}$, and $2 p^{2}\left({ }^{3} P\right) \varepsilon_{p}$.

\begin{tabular}{|c|c|c|c|c|c|c|c|c|c|c|c|}
\hline \multirow[b]{2}{*}{ State } & \multirow[b]{2}{*}{$E$ (a.u.) } & \multirow[b]{2}{*}{$E(\mathrm{eV})$} & \multirow[b]{2}{*}{$\Gamma$ (a.u.) } & \multirow[b]{2}{*}{$n^{*}$} & \multirow[b]{2}{*}{$\bar{\Gamma}$ (a.u.) } & \multicolumn{6}{|c|}{ Branching ratios $(\%)$} \\
\hline & & & & & & $\mathrm{ch}_{1}$ & $\mathrm{ch}_{2}$ & $\mathrm{ch}_{3}$ & $\mathrm{ch}_{4}$ & $\mathrm{ch}_{5}$ & $\mathrm{ch}_{6}$ \\
\hline $2 s 2 p\left({ }^{1} P\right) 3 d$ & -24.025619 & 7.58319 & $3.99[-4]$ & 2.983 & $1.06[-2]$ & 29.3 & 2.4 & 68.2 & & & \\
\hline $2 s 2 p\left({ }^{1} P\right) 4 d$ & -24.001114 & 8.24997 & $1.51[-4]$ & 3.972 & $9.44[-3]$ & 51.9 & 8.6 & 39.4 & & & \\
\hline $2 s 2 p\left({ }^{1} P\right) 5 d$ & -23.989687 & 8.56089 & $7.81[-5]$ & 4.966 & $9.56[-3]$ & 59.9 & 11.9 & 28.2 & & & \\
\hline $2 s 2 p\left({ }^{1} P\right) 6 d$ & -23.983475 & 8.72993 & $4.59[-5]$ & 5.964 & $9.73[-3]$ & 63.4 & 13.7 & 22.9 & & & \\
\hline $2 s 2 p\left({ }^{1} P\right) 7 d$ & -23.979732 & 8.83177 & $2.92[-5]$ & 6.962 & $9.86[-3]$ & 65.2 & 14.8 & 20.0 & & & \\
\hline $2 s 2 p\left({ }^{1} P\right) 8 d$ & -23.977306 & 8.89779 & $1.97[-5]$ & 7.961 & $9.96[-3]$ & 66.3 & 15.5 & 18.3 & & & \\
\hline $2 s 2 p\left({ }^{1} P\right) 9 d$ & -23.975645 & 8.94300 & $1.39[-5]$ & 8.960 & $1.00[-2]$ & 67.0 & 15.9 & 17.1 & & & \\
\hline $2 s 2 p\left({ }^{1} P\right) 10 d$ & -23.974457 & 8.97531 & $1.02[-5]$ & 9.959 & $1.01[-2]$ & 67.4 & 16.3 & 16.3 & & & \\
\hline $2 p^{3}$ & -24.084162 & 5.99025 & $2.50[-2]$ & 2.087 & $2.27[-1]$ & 3.0 & 8.4 & 88.6 & & & \\
\hline $2 s 2 p\left({ }^{1} P\right) 3 s$ & -24.090972 & 5.80494 & $1.56[-3]$ & 2.028 & $1.30[-2]$ & 63.1 & 4.5 & 32.4 & & & \\
\hline $2 s 2 p\left({ }^{1} P\right) 4 s$ & -24.023319 & 7.64577 & $3.47[-4]$ & 3.046 & $9.81[-3]$ & 86.6 & 13.1 & 0.3 & & & \\
\hline $2 s 2 p\left({ }^{1} P\right) 5 s$ & -23.999847 & 8.28445 & $1.52[-4]$ & 4.054 & $1.01[-2]$ & 87.8 & 11.6 & 0.5 & & & \\
\hline $2 s 2 p\left({ }^{1} P\right) 6 s$ & -23.988971 & 8.58039 & $8.00[-5]$ & 5.057 & $1.03[-2]$ & 88.4 & 11.0 & 0.7 & & & \\
\hline $2 s 2 p\left({ }^{1} P\right) 7 s$ & -23.983039 & 8.74179 & $4.70[-5]$ & 6.058 & $1.05[-2]$ & 88.6 & 10.6 & 0.8 & & & \\
\hline $2 s 2 p\left({ }^{1} P\right) 8 s$ & -23.979450 & 8.83946 & $2.99[-5]$ & 7.059 & $1.05[-2]$ & 88.8 & 10.4 & 0.8 & & & \\
\hline $2 s 2 p\left({ }^{1} P\right) 9 s$ & -23.977113 & 8.90304 & $2.02[-5]$ & 8.060 & $1.06[-2]$ & 88.9 & 10.2 & 0.9 & & & \\
\hline $2 s 2 p\left({ }^{1} P\right) 10 s$ & -23.975507 & 8.94673 & $1.43[-5]$ & 9.060 & $1.06[-2]$ & 89.0 & 10.2 & 0.9 & & & \\
\hline $2 s 2 p\left({ }^{1} P\right) 11 s$ & -23.974356 & 8.97805 & $1.04[-5]$ & 10.061 & $1.06[-2]$ & 89.0 & 10.1 & 0.9 & & & \\
\hline $2 s 2 p\left({ }^{1} P\right)$ & -23.969417 & 9.11247 & & & & & & & & & \\
\hline $2 p^{2}\left({ }^{3} P\right) 3 p$ & -23.930391 & 10.17436 & $1.53[-3]$ & 2.541 & $2.50[-2]$ & 1.5 & 27.2 & 28.4 & 2.9 & 40.0 & \\
\hline $2 p^{2}\left({ }^{1} D\right) 3 p$ & -23.912628 & 10.65768 & $1.50[-3]$ & 2.895 & $3.65[-2]$ & 0.4 & 16.5 & 48.0 & 16.8 & 18.4 & \\
\hline $2 p^{2}\left({ }^{3} P\right) 4 p$ & -23.890986 & 11.24655 & $1.06[-3]$ & 3.626 & $5.07[-2]$ & 0.8 & 29.7 & 35.3 & 0.2 & 34.0 & \\
\hline $2 p^{2}\left({ }^{1} D\right) 4 p$ & -23.878745 & 11.57965 & $9.63[-5]$ & 4.404 & $8.23[-3]$ & 1.8 & 9.8 & 1.6 & 69.0 & 17.7 & \\
\hline $2 p^{2}\left({ }^{3} P\right) 5 p$ & -23.874831 & 11.68614 & $5.88[-4]$ & 4.782 & $6.43[-2]$ & 0.4 & 25.7 & 41.0 & 9.2 & 23.7 & \\
\hline $2 p^{2}\left({ }^{3} P\right) 6 p$ & -23.868655 & 11.85420 & $3.03[-4]$ & 5.645 & $5.46[-2]$ & 0.6 & 33.2 & 32.7 & 0.8 & 32.7 & \\
\hline $2 p^{2}\left({ }^{1} D\right) 4 f$ & -23.868270 & 11.86465 & $7.94[-6]$ & 5.716 & $1.48[-3]$ & 0.1 & 13.1 & 61.3 & 2.0 & 23.6 & \\
\hline $2 p^{2}\left({ }^{3} P\right) 7 p$ & -23.864517 & 11.96679 & $1.30[-4]$ & 6.579 & $3.71[-2]$ & 0.8 & 35.7 & 25.4 & 0.7 & 37.3 & \\
\hline $2 p^{2}\left({ }^{1} D\right) 5 p$ & -23.862215 & 12.02941 & $2.99[-5]$ & 7.352 & $1.19[-2]$ & 1.7 & 2.5 & 10.6 & 77.6 & 7.6 & \\
\hline $2 p^{2}\left({ }^{3} P\right) 8 p$ & -23.861026 & 12.06178 & $1.46[-4]$ & 7.876 & $7.15[-2]$ & 0.3 & 24.0 & 41.6 & 15.9 & 18.3 & \\
\hline $2 p^{2}\left({ }^{3} P\right) 9 p$ & -23.859550 & 12.10194 & $1.12[-4]$ & 8.714 & $7.38[-2]$ & 0.4 & 31.5 & 36.6 & 4.2 & 27.4 & \\
\hline $2 p^{2}\left({ }^{3} P\right) 10 p$ & -23.858313 & 12.13559 & $7.32[-5]$ & 9.669 & $6.62[-2]$ & 0.5 & 33.8 & 33.6 & 1.8 & 30.3 & \\
\hline $2 p^{2}\left({ }^{3} P\right) 11 p$ & -23.857381 & 12.16095 & $4.79[-5]$ & 10.640 & $5.77[-2]$ & 0.7 & 36.8 & 29.0 & 1.2 & 32.4 & \\
\hline $2 p^{2}\left({ }^{1} D\right) 5 f$ & -23.857292 & 12.16336 & $4.82[-6]$ & 10.749 & $5.99[-3]$ & 0.2 & 8.0 & 68.9 & 4.6 & 18.2 & \\
\hline $2 p^{2}\left({ }^{3} P\right)$ & -23.852965 & 12.28111 & & & & & & & & & \\
\hline $2 p^{2}\left({ }^{1} D\right) 6 f$ & -23.851298 & 12.32646 & $2.78[-6]$ & 6.032 & $6.10[-4]$ & 1.3 & 3.2 & 41.1 & 11.7 & 3.0 & 39.7 \\
\hline $2 p^{2}\left({ }^{1} D\right) 7 f$ & -23.847670 & 12.42519 & $2.25[-6]$ & 7.031 & $7.81[-4]$ & 1.8 & 4.6 & 34.7 & 14.0 & 2.4 & 42.4 \\
\hline $2 p^{2}\left({ }^{1} D\right) 8 f$ & -23.845309 & 12.48943 & $1.75[-6]$ & 8.031 & $9.08[-4]$ & 2.2 & 5.0 & 33.1 & 16.5 & 2.3 & 40.9 \\
\hline $2 p^{2}\left({ }^{1} D\right) 9 f$ & -23.843688 & 12.53354 & $1.38[-6]$ & 9.030 & $1.02[-3]$ & 2.6 & 5.3 & 32.0 & 18.7 & 2.2 & 39.2 \\
\hline $2 p^{2}\left({ }^{1} D\right) 10 f$ & -23.842526 & 12.56515 & $1.10[-6]$ & 10.030 & $1.11[-3]$ & 3.0 & 5.5 & 31.1 & 20.6 & 2.1 & 37.7 \\
\hline $2 p^{2}\left({ }^{1} D\right) 7 p$ & -23.849196 & 12.38366 & $3.84[-4]$ & 6.554 & $1.08[-1]$ & 0.0 & 4.6 & 7.0 & 4.1 & 3.1 & 81.2 \\
\hline $2 p^{2}\left({ }^{1} D\right) 8 p$ & -23.846330 & 12.46166 & $2.61[-4]$ & 7.549 & $1.12[-1]$ & 0.0 & 4.5 & 6.4 & 3.3 & 3.0 & 82.8 \\
\hline $2 p^{2}\left({ }^{1} D\right) 9 p$ & -23.844401 & 12.51413 & $1.82[-4]$ & 8.546 & $1.14[-1]$ & 0.1 & 4.4 & 6.3 & 2.7 & 3.0 & 83.5 \\
\hline $2 p^{2}\left({ }^{1} D\right) 10 p$ & -23.843045 & 12.55104 & $1.32[-4]$ & 9.544 & $1.15[-1]$ & 0.1 & 4.4 & 6.2 & 2.3 & 2.9 & 84.1 \\
\hline $2 p^{2}\left({ }^{1} D\right) 11 p$ & -23.842055 & 12.57799 & $9.89[-5]$ & 10.543 & $1.16[-1]$ & 0.1 & 4.3 & 6.2 & 1.9 & 2.9 & 84.5 \\
\hline $2 p^{2}\left({ }^{1} D\right)$ & -23.837556 & 12.70039 & & & & & & & & & \\
\hline
\end{tabular}

one could guess for the third and sixth terms in the main series from the quantum defect of higher terms in the same series. As a consequence, the main and intruder states in these two pairs interact significantly with each other and cause a shift downward for the intruder energies and upward for the main-series terms, with respect to their projected position. Notice that the lowest term that emerges from the interaction within each pair has all but lost any propensity to decay to the $2 s 2 p\left({ }^{3} P\right) \varepsilon_{s}$ channel, whereas for the other autoionizing states with $2 p^{2}\left({ }^{1} D\right) n p$ dominant configurations, the branching ratios to the $2 s 2 p\left({ }^{3} P\right) \varepsilon_{s}$ and $2 s 2 p\left({ }^{1} P\right) \varepsilon_{s}$ channels are comparable. As a result, while all the resonances in this interval predominantly decay as $d$-wave electrons, the $2 p^{2}\left({ }^{1} D\right) 4 p$ and $2 p^{2}\left({ }^{1} D\right) 5 p$ states mostly decay as $s$ waves 
TABLE VII. Parameters of the ${ }^{2} P^{o}$ resonances located between the $2 p^{2}\left({ }^{1} D\right)$ and the $2 p^{2}\left({ }^{1} S\right)$ thresholds. The six channels that open in these first four energy intervals are, in order, $2 s^{2} \varepsilon_{p}, 2 s 2 p\left({ }^{3} P\right) \varepsilon_{s}, 2 s 2 p\left({ }^{3} P\right) \varepsilon_{d}, 2 s 2 p\left({ }^{1} P\right) \varepsilon_{s}, 2 s 2 p\left({ }^{1} P\right) \varepsilon_{d}, 2 p^{2}\left({ }^{3} P\right) \varepsilon_{p}, 2 p^{2}\left({ }^{1} D\right) \varepsilon_{p}$, and $2 p^{2}\left({ }^{1} D\right) \varepsilon_{f}$.

\begin{tabular}{|c|c|c|c|c|c|c|c|c|c|c|c|c|c|}
\hline \multirow[b]{2}{*}{ State } & \multirow[b]{2}{*}{$E$ (a.u.) } & \multirow[b]{2}{*}{$E(\mathrm{eV})$} & \multirow[b]{2}{*}{$\Gamma($ a.u. $)$} & \multirow[b]{2}{*}{$n^{*}$} & \multirow[b]{2}{*}{$\bar{\Gamma}$ (a.u.) } & \multicolumn{8}{|c|}{ Branching ratios (\%) } \\
\hline & & & & & & $\mathrm{ch}_{1}$ & $\mathrm{ch}_{2}$ & $\mathrm{ch}_{3}$ & $\mathrm{ch}_{4}$ & $\mathrm{ch}_{5}$ & $\mathrm{ch}_{6}$ & $\mathrm{ch}_{7}$ & $\mathrm{ch}_{8}$ \\
\hline 1 & -23.831819 & 12.85648 & $2.95[-3]$ & 2.139 & $2.89[-2]$ & 9.3 & 1.5 & 1.2 & 53.5 & 1.7 & 6.3 & 23.7 & 2.8 \\
\hline 2 & -23.813216 & 13.36267 & $3.17[-3]$ & 2.348 & $4.11[-2]$ & 7.5 & 38.1 & 17.3 & 4.3 & 7.6 & 7.2 & 7.8 & 10.1 \\
\hline 3 & -23.788597 & 14.03256 & $2.33[-3]$ & 2.752 & $4.86[-2]$ & 18.7 & 11.9 & 3.5 & 9.1 & 13.5 & 17.9 & 3.5 & 21.9 \\
\hline 4 & -23.765278 & 14.66707 & $1.13[-3]$ & 3.421 & $4.51[-2]$ & 0.2 & 12.7 & 24.6 & 5.9 & 0.6 & 21.5 & 5.2 & 29.3 \\
\hline 5 & -23.764580 & 14.68607 & $6.87[-4]$ & 3.450 & $2.82[-2]$ & 2.1 & 12.8 & 1.6 & 52.0 & 6.5 & 1.0 & 21.4 & 2.6 \\
\hline 6 & -23.748624 & 15.12024 & $4.57[-4]$ & 4.380 & $3.84[-2]$ & 3.3 & 8.0 & 18.8 & 25.0 & 0.9 & 17.1 & 2.8 & 24.0 \\
\hline 7 & -23.742951 & 15.27459 & $1.39[-4]$ & 4.952 & $1.69[-2]$ & 1.0 & 22.5 & 16.7 & 21.0 & 13.1 & 3.8 & 15.8 & 6.0 \\
\hline 8 & -23.740042 & 15.35375 & $2.11[-4]$ & 5.348 & $3.23[-2]$ & 9.8 & 7.4 & 15.4 & 35.3 & 1.8 & 11.3 & 3.7 & 15.3 \\
\hline 9 & -23.735232 & 15.48463 & $1.22[-4]$ & 6.282 & $3.01[-2]$ & 22.8 & 10.6 & 13.4 & 31.5 & 8.1 & 4.6 & 2.7 & 6.4 \\
\hline 10 & -23.732808 & 15.55059 & $5.07[-5]$ & 6.985 & $1.73[-2]$ & 18.8 & 15.1 & 7.2 & 0.3 & 52.3 & 4.3 & 0.9 & 1.2 \\
\hline 11 & -23.731994 & 15.57274 & $6.84[-5]$ & 7.280 & $2.64[-2]$ & 35.3 & 3.8 & 1.1 & 46.9 & 1.2 & 1.9 & 8.2 & 1.7 \\
\hline 12 & -23.730303 & 15.61873 & $1.16[-4]$ & 8.036 & $6.04[-2]$ & 37.1 & 5.3 & 0.7 & 8.6 & 14.0 & 14.1 & 7.1 & 13.2 \\
\hline 13 & -23.729010 & 15.65393 & $1.19[-4]$ & 8.805 & $8.15[-2]$ & 21.0 & 4.4 & 5.7 & 0.1 & 14.8 & 25.7 & 6.9 & 21.4 \\
\hline 14 & -23.727923 & 15.68352 & $7.73[-5]$ & 9.656 & $6.96[-2]$ & 9.1 & 4.3 & 11.1 & 2.6 & 14.7 & 32.0 & 6.3 & 19.9 \\
\hline 15 & -23.727043 & 15.70745 & $3.86[-5]$ & 10.561 & $4.54[-2]$ & 0.8 & 4.1 & 15.4 & 10.5 & 21.1 & 37.7 & 4.7 & 5.7 \\
\hline $2 p^{2}\left({ }^{1} S\right)$ & -23.722560 & 15.82943 & & & & & & & & & & & \\
\hline
\end{tabular}

instead $\left(78.8 \%\right.$ and $80.1 \%$ respectively). For the $2 p^{2}\left({ }^{1} D\right) 6 p$ intruder state, we estimate an apparent effective principal quantum number with respect to the $2 p^{2}\left({ }^{3} P\right)$ threshold of the order of $n^{*} \sim 25.5$, which is too close to threshold to be resolved in our calculation.

The narrow energy interval between the $2 p^{2}\left({ }^{3} P\right)$ and the $2 p^{2}\left({ }^{1} D\right)$ thresholds hosts two regular series, $2 p^{2}\left({ }^{1} D\right) n p$ and $2 p^{2}\left({ }^{1} D\right) n f$, with quite distinct character: The former decays predominantly to the $2 p^{2}\left({ }^{3} P\right) \varepsilon_{p}$ channel, while the latter is two orders of magnitude narrower and it decays in comparable proportions to the $2 s 2 p\left({ }^{3} P\right) \varepsilon_{d}$ and to the $2 p^{2}\left({ }^{3} P\right) \varepsilon_{p}$ channels.

The fifth and last energy interval, up to the $2 p^{2}\left({ }^{1} S\right)$ threshold, again features several intruder states owing to the small energy separation from the $2 s 3 s\left({ }^{3 / 1} S\right)$ nearby thresholds. In this case (see third panel from top in Fig. 3), the $2 s 3 s\left({ }^{3} S\right) 3-$ $6 p$ and the $2 s 3 s\left({ }^{1} s\right) 3-4 p$ intruder states interact strongly with the main $2 p^{2}\left({ }^{1} S\right) n p$ series. Indeed, the first 11 resonances in this interval are complex mixtures of terms from the main series and intruder series. As a consequence, no clear pattern is recognizable from either the resonance position or their

TABLE VIII. Parameters of the ${ }^{2} S^{e}$ resonances between the $2 s^{2}$ and the $2 s 2 p\left({ }^{3} P\right)$ thresholds.

\begin{tabular}{lccccc}
\hline \hline State & $E($ a.u. $)$ & $E(\mathrm{eV})$ & $\Gamma$ (a.u. $)$ & $n^{*}$ & $\bar{\Gamma}$ (a.u. $)$ \\
\hline $2 s 2 p\left({ }^{3} P\right) 3 p$ & -24.200990 & 2.81136 & $4.70[-4]$ & 2.733 & $9.59[-3]$ \\
$2 s 2 p\left({ }^{3} P\right) 4 p$ & -24.169861 & 3.65836 & $1.67[-4]$ & 3.736 & $8.69[-3]$ \\
$2 s 2 p\left({ }^{3} P\right) 5 p$ & -24.156336 & 4.02638 & $7.75[-5]$ & 4.736 & $8.23[-3]$ \\
$2 s 2 p\left({ }^{3} P\right) 6 p$ & -24.149244 & 4.21936 & $4.21[-5]$ & 5.736 & $7.95[-3]$ \\
$2 s 2 p\left({ }^{3} P\right) 7 p$ & -24.145067 & 4.33301 & $2.54[-5]$ & 6.735 & $7.77[-3]$ \\
$2 s 2 p\left({ }^{3} P\right) 8 p$ & -24.142402 & 4.40552 & $1.65[-5]$ & 7.735 & $7.64[-3]$ \\
$2 s 2 p\left({ }^{3} P\right) 9 p$ & -24.140599 & 4.45460 & $1.13[-5]$ & 8.735 & $7.54[-3]$ \\
$2 s 2 p\left({ }^{3} P\right) 10 p$ & -24.139321 & 4.48935 & $8.08[-6]$ & 9.735 & $7.45[-3]$ \\
$2 s 2 p\left({ }^{3} P\right)$ & -24.134046 & 4.63290 & & & \\
\hline \hline
\end{tabular}

branching ratio (compare with Table VII). A regular decay pattern for the main series emerges only above $n=8$.

\section{2. ${ }^{2} S^{e}$ autoionizing states}

The parameters of the resonances in ${ }^{2} S^{e}$ symmetry are reported in Tables VIII and IX. In the first energy interval, all the resonances belong to the regular $2 s 2 p\left({ }^{3} P\right) n p$ series. As mentioned in Sec. III C, in single-channel approximation the first term of this series, the $2 s 2 p\left({ }^{3} P\right) 2 p$ state, is above the $2 s^{2}$ ionization threshold. However, when the doubly occupied $2 p$ orbital is allowed to relax through interchannel interaction, the $2 s 2 p^{2}\left({ }^{2} S\right)$ state falls below the ionization threshold, thus giving rise to an additional state in the bound series, rather than to an autoionizing state.

As mentioned in the introduction to the present section, one experimental paper from 1976 claims to have measured the position of the $2 s 2 p\left({ }^{3} P\right) 3 p{ }^{2} S^{e}$ autoionizing state of the neutral boron atom [120]. The position calculated here for that state, $2.81136 \mathrm{eV}$ (compare with Table VIII), however, differs from the value $2.67701 \mathrm{eV}$ reported in [120] by as much as $134 \mathrm{meV}\left(\sim 1100 \mathrm{~cm}^{-1}\right)$. This apparent discrepancy is much larger than what one could expect on the basis of the excellent agreement already found for the position within the bound $2 s^{2} n s$ Rydberg series of the $2 s 2 p^{2}$ intruder state, which can be regarded as the very first term in the $2 s 2 p\left({ }^{3} P\right) n p$ series. In fact, the correct description of the $2 s 2 p^{2}$ state is arguably more challenging than that of the subsequent terms in the series. Furthermore, while the position of the $2 s 2 p^{2}$ is slightly affected by the mismatch between the experimental and theoretical separation of the $2 s^{2}$ and $2 s 2 p\left({ }^{3} P\right)$ thresholds, that of the $2 s 2 p\left({ }^{3} P\right) 3 p$ state is much less so, since we estimate the energy of the resonances using the $2 s 3 s\left({ }^{3} S\right)$ threshold as a reference. Indeed, the discrepancy between the experimental and the theoretical energy gap between the $2 s 2 p\left({ }^{3} P\right) 3 p$ and $2 s 3 s\left({ }^{3} S\right)$ is between just 1 and $4 \mathrm{meV}$, depending on which 
TABLE IX. Parameters of the ${ }^{2} S^{e}$. The decay channels are, in order, $2 s^{2} \varepsilon_{s}, 2 s 2 p\left({ }^{3} P\right) \varepsilon_{p}, 2 s 2 p\left({ }^{1} P\right) \varepsilon_{p}$, and $2 p^{2}\left({ }^{1} D\right) \varepsilon_{d}$.

\begin{tabular}{|c|c|c|c|c|c|c|c|c|c|}
\hline \multirow[b]{2}{*}{ State } & \multirow[b]{2}{*}{$E$ (a.u.) } & \multirow[b]{2}{*}{$E(\mathrm{eV})$} & \multirow[b]{2}{*}{$\Gamma$ (a.u.) } & \multirow[b]{2}{*}{$n^{*}$} & \multirow[b]{2}{*}{$\bar{\Gamma}$ (a.u.) } & \multicolumn{4}{|c|}{ Branching ratios (\%) } \\
\hline & & & & & & $\mathrm{ch}_{1}$ & $\mathrm{ch}_{2}$ & $\mathrm{ch}_{3}$ & $\mathrm{ch}_{4}$ \\
\hline $2 s 2 p\left({ }^{1} P\right) 3 p$ & -24.052112 & 6.86231 & $5.90[-3]$ & 2.459 & $8.78[-2]$ & 14.4 & 85.6 & & \\
\hline $2 s 2 p\left({ }^{1} P\right) 4 p$ & -24.010676 & 7.98980 & $2.17[-3]$ & 3.481 & $9.15[-2]$ & 16.8 & 83.2 & & \\
\hline $2 s 2 p\left({ }^{1} P\right) 5 p$ & -23.994237 & 8.43710 & $1.02[-3]$ & 4.488 & $9.18[-2]$ & 17.8 & 82.2 & & \\
\hline $2 s 2 p\left({ }^{1} P\right) 6 p$ & -23.985976 & 8.66187 & $7.94[-4]$ & 5.495 & $1.32[-1]$ & 18.4 & 81.6 & & \\
\hline $2 s 2 p\left({ }^{1} P\right) 7 p$ & -23.981286 & 8.78950 & $3.69[-4]$ & 6.490 & $1.01[-1]$ & 18.7 & 81.3 & & \\
\hline $2 s 2 p\left({ }^{1} P\right) 8 p$ & -23.978326 & 8.87004 & $2.39[-4]$ & 7.491 & $1.00[-1]$ & 18.9 & 81.1 & & \\
\hline $2 s 2 p\left({ }^{1} P\right) 9 p$ & -23.976350 & 8.92379 & $1.65[-4]$ & 8.492 & $1.01[-1]$ & 19.0 & 81.0 & & \\
\hline $2 s 2 p\left({ }^{1} P\right) 10 p$ & -23.974966 & 8.96146 & $1.19[-4]$ & 9.492 & $1.02[-1]$ & 19.1 & 80.9 & & \\
\hline $2 s 2 p\left({ }^{1} P\right)$ & -23.969417 & 9.11247 & & & & & & & \\
\hline $2 p^{2}\left({ }^{1} D\right) 3 d$ & -23.890814 & 11.25125 & $9.05[-5]$ & 3.064 & $2.60[-3]$ & 5.7 & 23.7 & 70.6 & \\
\hline $2 s 3 s^{2}$ & -23.877437 & 11.61522 & $2.43[-3]$ & 3.541 & $1.08[-1]$ & 30.4 & 7.1 & 62.6 & \\
\hline $2 p^{2}\left({ }^{1} D\right) 4 d$ & -23.868026 & 11.87129 & $1.34[-4]$ & 4.051 & $8.93[-3]$ & 10.3 & 19.2 & 70.5 & \\
\hline $2 p^{2}\left({ }^{1} D\right) 5 d$ & -23.857250 & 12.16452 & $5.19[-5]$ & 5.039 & $6.64[-3]$ & 9.6 & 30.9 & 59.5 & \\
\hline $2 p^{2}\left({ }^{1} D\right) 6 d$ & -23.851304 & 12.32630 & $2.84[-5]$ & 6.031 & $6.23[-3]$ & 9.6 & 42.0 & 48.3 & \\
\hline $2 p^{2}\left({ }^{1} D\right) 7 d$ & -23.847689 & 12.42467 & $1.80[-5]$ & 7.024 & $6.22[-3]$ & 9.4 & 51.9 & 38.7 & \\
\hline $2 p^{2}\left({ }^{1} D\right) 8 d$ & -23.845330 & 12.48886 & $1.24[-5]$ & 8.020 & $6.42[-3]$ & 9.0 & 59.7 & 31.4 & \\
\hline $2 p^{2}\left({ }^{1} D\right) 9 d$ & -23.843707 & 12.53302 & $9.15[-6]$ & 9.016 & $6.71[-3]$ & 8.5 & 65.4 & 26.1 & \\
\hline $2 p^{2}\left({ }^{1} D\right) 10 d$ & -23.842543 & 12.56469 & $7.00[-6]$ & 10.013 & $7.03[-3]$ & 8.1 & 69.5 & 22.5 & \\
\hline $2 p^{2}\left({ }^{1} D\right)$ & -23.837556 & 12.70039 & & & & & & & \\
\hline $2 p^{2}\left({ }^{1} S\right) 3 s$ & -23.825799 & 13.02029 & $8.27[-3]$ & 2.201 & $8.81[-2]$ & 0.1 & 30.9 & 21.7 & 47.4 \\
\hline $2 p^{2}\left({ }^{1} S\right) 4 s$ & -23.776690 & 14.35655 & $2.87[-3]$ & 3.039 & $8.06[-2]$ & 3.1 & 33.7 & 38.2 & 25.0 \\
\hline $2 s 3 s\left({ }^{3} S\right) 4 s$ & -23.774563 & 14.41444 & $2.99[-4]$ & 3.101 & $8.90[-3]$ & 39.7 & 42.8 & 9.0 & 8.5 \\
\hline $2 s 3 p^{2}$ & -23.757395 & 14.88157 & $3.17[-3]$ & 3.789 & $1.72[-1]$ & 7.0 & 66.2 & 9.4 & 17.4 \\
\hline $2 p^{2}\left({ }^{1} S\right) 5 s$ & -23.753330 & 14.99217 & $2.38[-3]$ & 4.031 & $1.56[-1]$ & 5.0 & 20.8 & 38.7 & 35.5 \\
\hline $2 s 3 s\left({ }^{3} S\right) 5 s$ & -23.746728 & 15.17182 & $4.09[-5]$ & 4.548 & $3.85[-3]$ & 48.3 & 6.1 & 29.5 & 16.0 \\
\hline $2 s 3 s\left({ }^{1} S\right) 4 s$ & -23.744557 & 15.23091 & $4.29[-4]$ & 4.768 & $4.65[-2]$ & 18.0 & 46.0 & 32.5 & 3.5 \\
\hline $2 p^{2}\left({ }^{1} S\right) 6 s$ & -23.740248 & 15.34815 & $5.88[-4]$ & 5.317 & $8.84[-2]$ & 0.7 & 43.2 & 17.2 & 38.9 \\
\hline $2 p^{2}\left({ }^{1} S\right) 7 s$ & -23.735707 & 15.47171 & $4.48[-4]$ & 6.167 & $1.05[-1]$ & 0.8 & 25.3 & 36.1 & 37.7 \\
\hline $2 s 3 s\left({ }^{3} S\right) 6 s$ & -23.733990 & 15.51841 & $8.88[-5]$ & 6.614 & $2.57[-2]$ & 30.7 & 55.7 & 10.3 & 3.3 \\
\hline $2 p^{2}\left({ }^{1} S\right) 8 s$ & -23.732409 & 15.56145 & $2.81[-4]$ & 7.125 & $1.02[-1]$ & 1.3 & 23.0 & 40.1 & 35.6 \\
\hline $2 p^{2}\left({ }^{1} S\right) 9 s$ & -23.730171 & 15.62235 & $1.83[-4]$ & 8.105 & $9.76[-2]$ & 1.8 & 21.2 & 43.1 & 34.0 \\
\hline $2 p^{2}\left({ }^{1} S\right) 10 s$ & -23.728606 & 15.66492 & $1.26[-4]$ & 9.094 & $9.49[-2]$ & 2.0 & 20.5 & 44.5 & 33.0 \\
\hline $2 p^{2}\left({ }^{1} S\right) 11 s$ & -23.727477 & 15.69563 & $8.26[-5]$ & 10.084 & $8.47[-2]$ & 7.3 & 48.3 & 16.2 & 28.2 \\
\hline $2 s 3 s\left({ }^{3} S\right) 7 s$ & -23.727468 & 15.69590 & $4.85[-5]$ & 10.094 & $4.98[-2]$ & 35.1 & 30.4 & 27.8 & 6.6 \\
\hline $2 p^{2}\left({ }^{1} S\right)$ & -23.722560 & 15.82943 & & & & & & & \\
\hline
\end{tabular}

values of $J$ one considers. For these reasons, the present calculated result for the position of this resonance should not be off the mark by more than $\simeq 200 \mathrm{~cm}^{-1}$.

To ascertain the convergence of our results, we carried out a larger calculation, using extended and thicker series of knots for the $B$ splines, more stringent thresholds for the determination of the LC basis, and including five additional virtual PWCs: $2 s 4 d\left({ }^{1,3} D\right) \varepsilon_{d}, 2 s 4 f\left({ }^{1,3} F\right) \varepsilon_{f}, 2 s 4 p\left({ }^{1} P\right) \varepsilon_{p}$. The new position of the $2 s 2 p\left({ }^{3} P\right) 3 p$ resonance, however, was found to differ from the original one by just few $\mathrm{cm}^{-1}$. To exclude that the discrepancy between the present theoretical result and the value reported in [120] might be due to a highly asymmetric photoabsorption profile, we computed the cross section of the neutral boron atom from the $2 s^{2} 2 p$ ground state in a small energy range close to the resonance in both length and velocity gauge. As shown in Fig. 4, the $2 s 2 p 3 p$ resonance gives rise to a sharp peak at -24.201046 a.u., which differs by a mere $1.5 \mathrm{meV}$ from the pole of the scattering matrix, at -24.200990 a.u. Moreover, the good agreement between the two gauges supports our opinion that the calculated cross section reliably reproduces the absorption spectrum of the atom. In conclusion, the position assigned to the $2 s 2 p\left({ }^{3} P\right) 3 p{ }^{2} S^{e}$ resonance in [120], which, to the best of our knowledge, is the only one available in the literature, is irreconcilable with the present theoretical result, and deserves further experimental investigation.

In the second interval, between the $2 s 2 p\left({ }^{3} P\right)$ and the $2 s 2 p\left({ }^{1} P\right)$ thresholds, the $2 s 2 p\left({ }^{1} P\right) n p$ series is also regular and it decays preferentially to the $2 s 2 p\left({ }^{3} P\right) \varepsilon_{p}$ channel, a process that does not require the parent ion to change configuration or the satellite electron to change angular momentum. In fact, the reduced width of this series, $\bar{\Gamma} \simeq 10^{-2}$ a.u., is the largest of this energy region among all natural-parity doublet resonances, with the only exception of the $2 p^{3}\left({ }^{2} P\right)$ state.

In the third interval, between the $2 s 2 p\left({ }^{1} P\right)$ and the $2 p^{2}\left({ }^{1} D\right)$ thresholds, we encounter the first autoionizing intruder state 


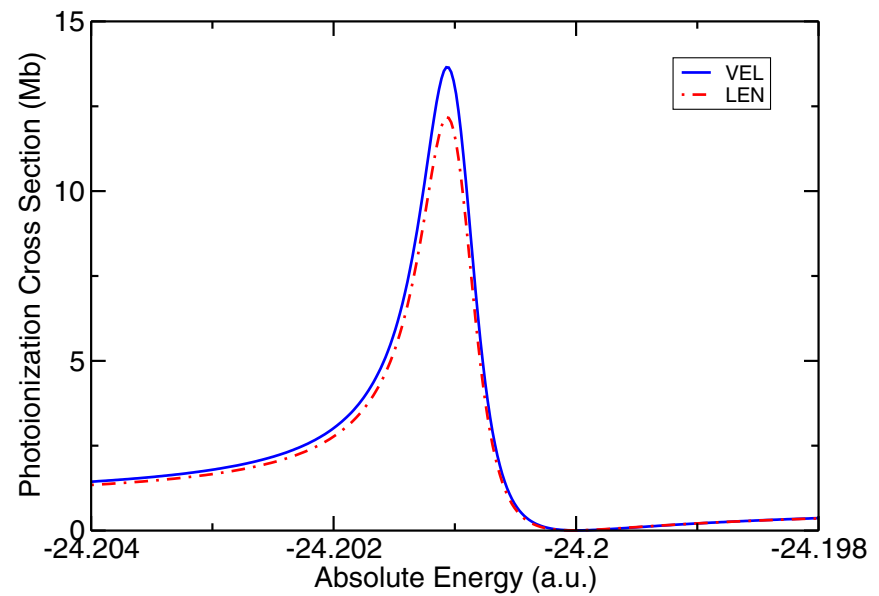

FIG. 4. Photoionization cross section of the boron atom from the $2 s^{2} 2 p{ }^{2} P^{o}$ ground state to the $1 s^{2} \varepsilon_{s}$ ionization continuum, in proximity of the $2 s 2 p\left({ }^{3} P\right) 3 p\left({ }^{2} S\right)$ autoionizing state, computed in velocity gauge (blue solid line) and length gauge (red dash-dotted line). The cross section has been convoluted with a FWHM $=5 \mathrm{meV}$ Gaussian.

of the ${ }^{2} S^{e}$ manifold, with dominant configuration $2 s 3 s^{2}$. As already observed for the $2 s 2 p^{2}\left({ }^{2} S\right)$ state, even in this case the multiple occupancy of an excited orbital results in a significant shift of the energy of the $2 s 3 s\left({ }^{3} S\right) 3 s$ single-channel approximation to the state, once the state is allowed to relax in the CI calculation. Indeed, the intruder is clearly recognizable as the large resonance between the first and the second terms of the $2 p^{2}\left({ }^{1} D\right)$ nd series (compare with the fourth panel from the top in Fig. 3). This conclusion is also confirmed by the analysis of the resonance composition in terms of Rydberg PWC states, which shows that while the first autoionizing state in this energy region is characterized by a large contribution from the $2 p^{2}\left({ }^{1} D\right) 3 d$ single-channel Rydberg state, the next autoionizing state has a distinct $2 s 3 s^{2}$ character. The branching ratio of the higher $2 p^{2}\left({ }^{1} D\right) n d$ states drifts steadily from the $2 s 2 p\left({ }^{1} P\right) \varepsilon_{p}$ to the $2 s 2 p\left({ }^{3} P\right) \varepsilon_{p}$ channel, either as a long-range consequence of the $2 s 3 s^{2}$ intruder state or as a general feature of the highly polarizable $2 s 2 p^{2}$ parent ion.

The fourth energy interval, between the $2 p^{2}\left({ }^{1} D\right)$ and the $2 p^{2}\left({ }^{1} S\right)$ thresholds, features, beyond the main $2 p^{2}\left({ }^{1} S\right) n s$ series, as many as six intruders states of diverse character: four from the very close $2 s 3 s\left({ }^{3} S\right) n s$ series $(n=4-7)$, as well as the $2 s 3 s\left({ }^{3} S\right) 4 s$ and the $2 s 3 p^{2}$ states. By looking at the fifth panel in Fig. 3, the terms of the principal series are recognizable as light-blue peaks with comparatively large width, $\bar{\Gamma} \simeq 0.1$ a.u., while the intruders from the $2 s 3 s\left({ }^{3} S\right) n s$ series give rise to narrower peaks with a characteristically small propensity to decay to the $2 p^{2}\left({ }^{1} D\right) \varepsilon_{d}$ channel. The latter circumstance is reasonable, given that the $\mathrm{B}^{* *}(2 s 3 s n s) \rightarrow \mathrm{B}^{+}\left[2 p^{2}\left({ }^{1} D\right)\right]+e_{d}^{-}$ decay path requires all three orbitals in the initial dominant configuration to change and the parent ion to exchange as much as two quanta of angular momentum with the bound $n s$ satellite in order to release it to the continuum as a $d$ wave. On the other hand, the assignment of the fourth resonance, with $n^{*}=3.789$, from the resonant scattering parameters alone, is not obvious. In this case, the analysis of the resonance composition in terms of its Rydberg components is useful to establish that this resonance is essentially due to the $2 s 3 p^{2}$

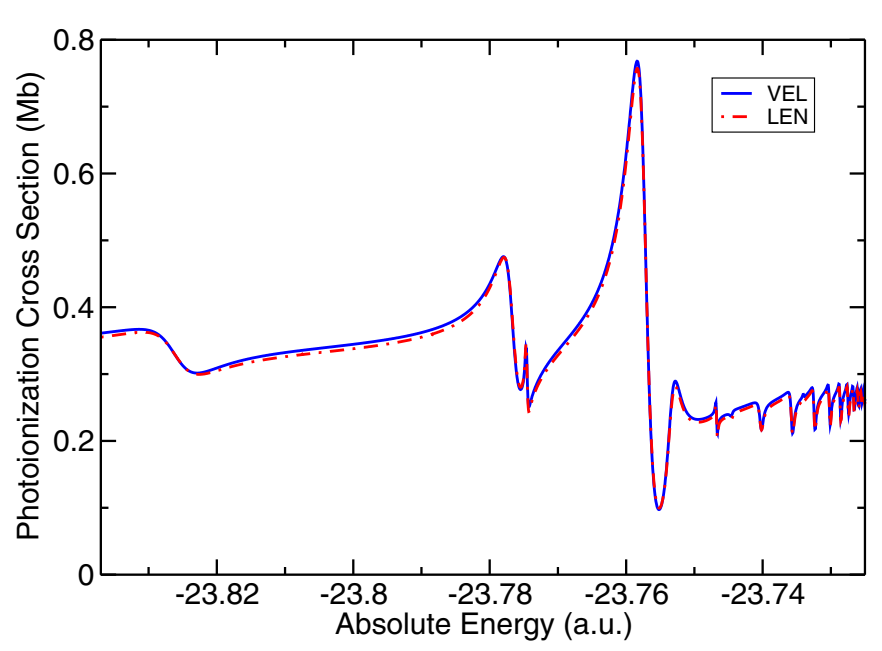

FIG. 5. Total photoionization cross section of the boron atom from the $2 s^{2} 2 p$ ground state to the ${ }^{2} S^{e}$ ionization continua, between the $2 p^{2}\left({ }^{1} D\right)$ and the $2 p^{2}\left({ }^{1} S\right)$ thresholds. See Fig. 4 and text for further details.

intruder state. Once again, the final energy of this state, in which the parent ion binds an electron in an orbital that is already occupied, is significantly lower than the value obtained for the $2 s 3 p\left({ }^{3} P^{o}\right) 3 p$ state in the frozen-ion approximation. The two resonances with $n^{*}$ close to 10 originate from the $2 p^{2}\left({ }^{1} S\right) 11 s$ term of the main series and the $2 s 3 s\left({ }^{3} S\right) 7 s$ intruder state. Even if these two states have extremely close unperturbed energies, -23.7274382 and -23.7266211 a.u., and indeed they give rise to two virtually overlapping resonances, their widths, and even more the small propensity of the narrowest to decay to the $2 p^{2}\left({ }^{1} D\right) \varepsilon_{d}$ channel, indicates that the resonance at $n^{*}=10.084$ belongs in fact to the main series, while the other still has marked intruder character. All the remaining resonances in the interval belong to the $2 p^{2}\left({ }^{1} S\right) n s$ series. The distinction of the resonances due to intruder states from those of the main series is also visible from their profile in the total photoionization cross section from the $2 s^{2} 2 p$ ground state to the ${ }^{2} S^{e}$ continuum, in the fourth energy interval, which is shown in Fig. 5. The cross section is dominated by the strong peak of the $2 s 3 p^{2}$ intruder state, at -23.757 a.u., which also alters dramatically the profile of the nearest terms of the main $2 p^{2}\left({ }^{1} S\right) n s$ series. The narrow $2 s 3 s\left({ }^{3} S\right) n s$ intruders, instead, are barely visible.

TABLE $X$. Parameters of the ${ }^{2} P^{e}$ resonances between the $2 s 2 p\left({ }^{3} P\right)$ and the $2 s 2 p\left({ }^{1} P\right)$ thresholds.

\begin{tabular}{lccccc}
\hline \hline State & $E$ (a.u.) & $E(\mathrm{eV})$ & $\Gamma$ (a.u.) & $n^{*}$ & $\bar{\Gamma}$ (a.u.) \\
\hline $2 s 2 p\left({ }^{1} P\right) 3 p$ & -24.052545 & 6.85054 & $1.88[-3]$ & 2.453 & $2.77[-2]$ \\
$2 s 2 p\left({ }^{1} P\right) 4 p$ & -24.011304 & 7.97271 & $2.46[-4]$ & 3.455 & $1.01[-2]$ \\
$2 s 2 p\left({ }^{1} P\right) 5 p$ & -23.994697 & 8.42457 & $3.57[-5]$ & 4.447 & $3.14[-3]$ \\
$2 s 2 p\left({ }^{1} P\right) 6 p$ & -23.986322 & 8.65246 & $2.87[-6]$ & 5.438 & $4.62[-4]$ \\
$2 s 2 p\left({ }^{1} P\right) 7 p$ & -23.981507 & 8.78349 & $1.27[-7]$ & 6.431 & $3.39[-5]$ \\
$2 s 2 p\left({ }^{1} P\right) 8 p$ & -23.978483 & 8.86576 & $1.80[-6]$ & 7.426 & $7.37[-4]$ \\
$2 s 2 p\left({ }^{1} P\right) 9 p$ & -23.976458 & 8.92086 & $3.06[-6]$ & 8.427 & $1.83[-3]$ \\
$\mathbf{2 s} \mathbf{2 p}\left({ }^{1} \boldsymbol{P}\right)$ & -23.969417 & 9.11247 & & & \\
\hline \hline
\end{tabular}


TABLE XI. Parameters of the ${ }^{2} P^{e}$ resonances between the $2 s 2 p\left({ }^{1} P\right)$ and the $2 s 3 p\left({ }^{3} P\right)$ thresholds. The decay channels are, in order, $2 s 2 p\left({ }^{3} P\right) \varepsilon_{p}, 2 s 2 p\left({ }^{1} P\right) \varepsilon_{p}, 2 p^{2}\left({ }^{3} P\right) \varepsilon_{s}, 2 p^{2}\left({ }^{3} P\right) \varepsilon_{d}$, and $2 p^{2}\left({ }^{1} D\right) \varepsilon_{d}$.

\begin{tabular}{|c|c|c|c|c|c|c|c|c|c|c|}
\hline \multirow[b]{2}{*}{ State } & \multirow[b]{2}{*}{$E$ (a.u.) } & \multirow[b]{2}{*}{$E(\mathrm{eV})$} & \multirow[b]{2}{*}{$\Gamma$ (a.u.) } & \multirow[b]{2}{*}{$n^{*}$} & \multirow[b]{2}{*}{$\bar{\Gamma}$ (a.u.) } & \multicolumn{5}{|c|}{ Branching ratios (\%) } \\
\hline & & & & & & $\mathrm{ch}_{1}$ & $\mathrm{ch}_{2}$ & $\mathrm{ch}_{3}$ & $\mathrm{ch}_{4}$ & $\overline{\mathrm{ch}_{5}}$ \\
\hline $2 p^{2}\left({ }^{3} P\right) 3 s$ & -23.957312 & 9.44183 & $1.86[-2]$ & 2.189 & $1.95[-1]$ & 49.6 & 50.4 & & & \\
\hline $2 p^{2}\left({ }^{3} P\right) 4 s$ & -23.901645 & 10.95654 & $5.64[-3]$ & 3.205 & $1.86[-1]$ & 52.1 & 47.9 & & & \\
\hline $2 p^{2}\left({ }^{3} P\right) 5 s$ & -23.881180 & 11.51338 & $2.44[-3]$ & 4.210 & $1.82[-1]$ & 52.8 & 47.2 & & & \\
\hline $2 p^{2}\left({ }^{3} P\right) 6 s$ & -23.871373 & 11.78023 & $1.28[-3]$ & 5.212 & $1.81[-1]$ & 53.6 & 46.4 & & & \\
\hline $2 p^{2}\left({ }^{3} P\right) 7 s$ & -23.865918 & 11.92865 & $7.51[-4]$ & 6.213 & $1.80[-1]$ & 53.4 & 46.6 & & & \\
\hline $2 p^{2}\left({ }^{3} P\right) 8 s$ & -23.862574 & 12.01966 & $4.78[-4]$ & 7.213 & $1.79[-1]$ & 53.7 & 46.3 & & & \\
\hline $2 p^{2}\left({ }^{3} P\right) 9 s$ & -23.860376 & 12.07947 & $3.23[-4]$ & 8.214 & $1.79[-1]$ & 53.9 & 46.1 & & & \\
\hline $2 p^{2}\left({ }^{3} P\right) 10 s$ & -23.858854 & 12.12088 & $2.29[-4]$ & 9.214 & $1.79[-1]$ & 54.2 & 45.8 & & & \\
\hline $2 p^{2}\left({ }^{3} P\right) 11 s$ & -23.857757 & 12.15072 & $1.67[-4]$ & 10.214 & $1.78[-1]$ & 56.9 & 43.1 & & & \\
\hline $2 p^{2}\left({ }^{1} D\right) 3 d$ & -23.892882 & 11.19496 & $1.79[-4]$ & 3.539 & $7.93[-3]$ & 35.7 & 64.3 & & & \\
\hline $2 p^{2}\left({ }^{1} D\right) 4 d$ & -23.868935 & 11.84658 & $9.91[-5]$ & 5.595 & $1.74[-2]$ & 35.4 & 64.6 & & & \\
\hline $2 p^{2}\left({ }^{1} D\right) 5 d$ & -23.857627 & 12.15425 & $4.25[-5]$ & 10.356 & $4.72[-2]$ & 36.5 & 63.5 & & & \\
\hline $2 p^{2}\left({ }^{3} P\right) 3 d$ & -23.912285 & 10.66702 & $1.33[-4]$ & 2.903 & $3.26[-3]$ & 13.1 & 86.9 & & & \\
\hline $2 p^{2}\left({ }^{3} P\right) 4 d$ & -23.885793 & 11.38787 & $4.55[-5]$ & 3.903 & $2.71[-3]$ & 11.7 & 88.3 & & & \\
\hline $2 p^{2}\left({ }^{3} P\right) 5 d$ & -23.873862 & 11.71251 & $4.23[-5]$ & 4.891 & $4.96[-3]$ & 18.1 & 81.9 & & & \\
\hline $2 p^{2}\left({ }^{3} P\right) 6 d$ & -23.867283 & 11.89151 & $6.62[-6]$ & 5.909 & $1.37[-3]$ & 4.6 & 95.4 & & & \\
\hline $2 p^{2}\left({ }^{3} P\right) 7 d$ & -23.863481 & 11.99497 & $1.16[-5]$ & 6.895 & $3.80[-3]$ & 15.4 & 84.6 & & & \\
\hline $2 p^{2}\left({ }^{3} P\right) 8 d$ & -23.860995 & 12.06261 & $9.83[-6]$ & 7.891 & $4.83[-3]$ & 17.7 & 82.3 & & & \\
\hline $2 p^{2}\left({ }^{3} P\right) 9 d$ & -23.859299 & 12.10877 & $9.08[-6]$ & 8.885 & $6.37[-3]$ & 20.2 & 79.8 & & & \\
\hline $2 p^{2}\left({ }^{3} P\right) 10 d$ & -23.858106 & 12.14121 & $1.45[-5]$ & 9.861 & $1.39[-2]$ & 26.4 & 73.6 & & & \\
\hline $2 p^{2}\left({ }^{3} P\right)$ & -23.852965 & 12.28111 & & & & & & & & \\
\hline $2 p^{2}\left({ }^{1} D\right) 6 d$ & -23.851501 & 12.32093 & $8.89[-5]$ & 5.988 & $1.91[-2]$ & 13.9 & 20.6 & 1.5 & 64.0 & \\
\hline $2 p^{2}\left({ }^{1} D\right) 7 d$ & -23.847799 & 12.42167 & $5.79[-5]$ & 6.987 & $1.97[-2]$ & 13.6 & 20.1 & 1.5 & 64.9 & \\
\hline $2 p^{2}\left({ }^{1} D\right) 8 d$ & -23.845397 & 12.48705 & $3.94[-5]$ & 7.986 & $2.01[-2]$ & 13.5 & 19.9 & 1.4 & 65.2 & \\
\hline $2 p^{2}\left({ }^{1} D\right) 9 d$ & -23.843749 & 12.53187 & $2.80[-5]$ & 8.985 & $2.03[-2]$ & 13.4 & 19.8 & 1.4 & 65.4 & \\
\hline $2 p^{2}\left({ }^{1} D\right) 10 d$ & -23.842571 & 12.56392 & $2.06[-5]$ & 9.985 & $2.05[-2]$ & 13.4 & 19.7 & 1.4 & 65.5 & \\
\hline $2 p^{2}\left({ }^{1} D\right)$ & -23.837556 & 12.70039 & & & & & & & & \\
\hline $2 s 3 p\left({ }^{3} P\right) 4 p$ & -23.707441 & 16.24082 & $3.65[-5]$ & 2.906 & $8.97[-4]$ & 54.6 & 6.3 & 24.3 & 0.4 & 14.4 \\
\hline $2 s 3 p\left({ }^{3} P\right) 5 p$ & -23.680565 & 16.97213 & $1.13[-4]$ & 3.933 & $6.85[-3]$ & 26.3 & 1.6 & 14.8 & 7.1 & 50.2 \\
\hline $2 s 3 p\left({ }^{3} P\right) 6 p$ & -23.668253 & 17.30711 & $1.74[-5]$ & 4.998 & $2.18[-3]$ & 56.4 & 4.9 & 24.8 & 2.7 & 11.2 \\
\hline $2 s 3 p\left({ }^{3} P\right) 7 p$ & -23.662086 & 17.47493 & $1.53[-5]$ & 6.010 & $3.33[-3]$ & 56.6 & 10.2 & 26.3 & 1.1 & 5.8 \\
\hline $2 s 3 p\left({ }^{3} P\right) 8 p$ & -23.658407 & 17.57504 & $1.32[-5]$ & 7.013 & $4.57[-3]$ & 53.4 & 14.1 & 26.3 & 0.7 & 5.5 \\
\hline $2 s 3 p\left({ }^{3} P\right) 9 p$ & -23.656028 & 17.63976 & $1.24[-5]$ & 8.013 & $6.39[-3]$ & 51.9 & 16.1 & 28.1 & 0.3 & 3.7 \\
\hline $2 s 3 p\left({ }^{3} P\right) 10 p$ & -23.654386 & 17.68445 & $1.25[-5]$ & 9.021 & $9.21[-3]$ & 41.6 & 26.4 & 22.8 & 0.9 & 8.3 \\
\hline $2 s 3 p\left({ }^{3} P\right) 11 p$ & -23.653220 & 17.71618 & $8.45[-6]$ & 10.021 & $8.51[-3]$ & 44.3 & 23.8 & 23.7 & 0.7 & 7.5 \\
\hline $2 s 3 p^{2}$ & -23.776131 & 14.37177 & $4.16[-3]$ & 1.977 & $3.22[-2]$ & 10.1 & 57.3 & 13.6 & 0.4 & 18.6 \\
\hline $2 s 3 p\left({ }^{1} P\right) 4 p$ & -23.699179 & 16.46562 & $1.26[-3]$ & 3.133 & $3.88[-2]$ & 14.3 & 59.9 & 13.9 & 0.4 & 11.4 \\
\hline $2 s 3 p\left({ }^{1} P\right) 5 p$ & -23.676933 & 17.07094 & $6.12[-4]$ & 4.175 & $4.45[-2]$ & 11.1 & 53.2 & 12.0 & 1.6 & 22.1 \\
\hline $2 s 3 p\left({ }^{1} P\right) 6 p$ & -23.666647 & 17.35081 & $2.79[-4]$ & 5.212 & $3.95[-2]$ & 15.2 & 58.6 & 13.3 & 0.6 & 12.3 \\
\hline $2 s 3 p\left({ }^{1} P\right) 7 p$ & -23.660960 & 17.50557 & $1.55[-4]$ & 6.270 & $3.81[-2]$ & 15.4 & 58.9 & 13.1 & 0.5 & 12.1 \\
\hline $2 s 3 p\left({ }^{1} P\right) 8 p$ & -23.657497 & 17.59979 & $9.01[-5]$ & 7.350 & $3.58[-2]$ & 16.1 & 59.2 & 13.3 & 0.4 & 11.0 \\
\hline $2 s 3 p\left({ }^{1} P\right) 9 p$ & -23.655180 & 17.66284 & $6.08[-5]$ & 8.489 & $3.72[-2]$ & 10.2 & 58.2 & 11.1 & 1.5 & 19.0 \\
\hline $2 s 3 p\left({ }^{1} P\right) 10 p$ & -23.653655 & 17.70434 & $4.95[-5]$ & 9.610 & $4.39[-2]$ & 12.4 & 60.0 & 11.9 & 0.8 & 14.9 \\
\hline $2 s 3 d^{2}$ & -23.677786 & 17.04774 & $2.26[-4]$ & 4.114 & $1.57[-2]$ & 7.6 & 3.1 & 5.6 & 14.1 & 69.6 \\
\hline $2 s 3 d\left({ }^{3} D\right) 4 d$ & -23.655767 & 17.64686 & $2.39[-5]$ & 8.151 & $1.29[-2]$ & 19.7 & 2.1 & 25.9 & 17.9 & 34.4 \\
\hline $2 s 3 p\left({ }^{3} P\right)$ & -23.648241 & 17.85165 & & & & & & & & \\
\hline
\end{tabular}

\section{3. ${ }^{2} \boldsymbol{P}^{e}$ autoionizing states}

The parameters for the ${ }^{2} P^{e}$ resonances are given in Tables X and XI. The first interval, which for this manifold is comprised between the $2 s 2 p\left({ }^{3} P\right)$ and the $2 s 2 p\left({ }^{1} P\right)$ thresholds, contains the $2 s 2 p\left({ }^{1} P\right) n p$ series of autoionizing states and no intruder states. While the effective quantum numbers of the terms in this series seem quite regular, however, the reduced width $\bar{\Gamma}_{n}$ exhibits a smooth yet very pronounced energy dependence, with a deep minimum in correspondence of the $2 s 2 p\left({ }^{1} P\right) 7 s$ states, for which $\bar{\Gamma}$ is almost three orders of magnitude smaller than that of the first term, followed by a steady recovery afterwards. This behavior is readily recognized as the hallmark 


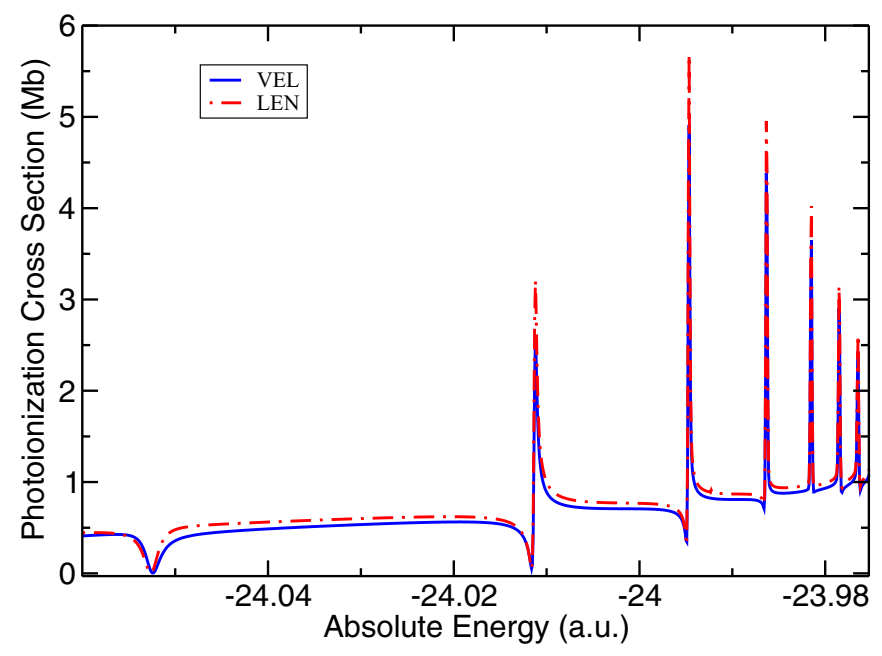

FIG. 6. Photoionization cross section of the boron atom from the $2 s^{2} 2 p{ }^{2} P^{o}$ ground state to the $2 s 2 p\left({ }^{3} P\right) \varepsilon_{p}\left({ }^{2} P\right)$ ionization continuum. See Fig. 4 and text for further details.

of the interaction with an intruder state, where the minimum in the reduced width results from the destructive interference between the decay amplitude of the main series and that of the contaminant configuration, as predicted by quantum-defect theory [118].

Such a mechanism, which is similar to the one responsible for the zero of a Fano profile in photoionization cross sections, is reflected here also in the inversion of the asymmetry parameter of the resonance profiles in the photoionization from the $2 s^{2} 2 p$ ground state, shown in Fig. 6. This prediction is confirmed by the presence, in the second energy interval, of the $2 p^{2}\left({ }^{3} P\right) 3 s$ resonance, which is located just $220 \mathrm{meV}$ above the $2 s 2 p\left({ }^{1} P\right)$ threshold and has a width as large as $506 \mathrm{meV}$.

In the second energy interval, between the $2 s 2 p\left({ }^{1} P\right)$ and the $2 p^{2}\left({ }^{3} P\right)$ thresholds, there are two main series, the broad $2 p^{2}\left({ }^{3} P\right) n s$ states $(\bar{\Gamma} \simeq 0.2$ a.u. $)$ and the narrower $2 p^{2}\left({ }^{3} P\right) n d$

TABLE XII. Parameters of the ${ }^{2} D^{e}$ resonances in the first energy interval, between the $2 s^{2}$ and the $2 s 2 p\left({ }^{3} P\right)$ thresholds.

\begin{tabular}{lccccc}
\hline \hline State & $E$ (a.u.) & $E(\mathrm{eV})$ & $\Gamma$ (a.u. $)$ & $n^{*}$ & $\bar{\Gamma}$ (a.u.) \\
\hline $2 s 2 p\left({ }^{3} P\right) 3 p$ & -24.209873 & 2.56964 & $1.74[-3]$ & 2.568 & $2.94[-2]$ \\
$2 s 2 p\left({ }^{3} P\right) 4 p$ & -24.172940 & 3.57460 & $5.68[-4]$ & 3.585 & $2.62[-2]$ \\
$2 s 2 p\left({ }^{3} P\right) 5 p$ & -24.157826 & 3.98584 & $2.87[-4]$ & 4.585 & $2.77[-2]$ \\
$2 s 2 p\left({ }^{3} P\right) 6 p$ & -24.150061 & 4.19713 & $1.54[-4]$ & 5.588 & $2.69[-2]$ \\
$2 s 2 p\left({ }^{3} P\right) 7 p$ & -24.145566 & 4.31945 & $9.34[-5]$ & 6.588 & $2.67[-2]$ \\
$2 s 2 p\left({ }^{3} P\right) 8 p$ & -24.142730 & 4.39662 & $6.12[-5]$ & 7.588 & $2.68[-2]$ \\
$2 s 2 p\left({ }^{3} P\right) 9 p$ & -24.140826 & 4.44842 & $4.25[-5]$ & 8.588 & $2.69[-2]$ \\
$2 s 2 p\left({ }^{3} P\right) 10 p$ & -24.139486 & 4.48488 & $3.09[-5]$ & 9.587 & $2.72[-2]$ \\
$2 s 2 p\left({ }^{3} P\right) 4 f$ & -24.165149 & 3.78659 & $1.57[-7]$ & 4.009 & $1.02[-5]$ \\
$2 s 2 p\left({ }^{3} P\right) 5 f$ & -24.153977 & 4.09057 & $1.19[-7]$ & 5.009 & $1.49[-5]$ \\
$2 s 2 p\left({ }^{3} P\right) 6 f$ & -24.147897 & 4.25601 & $8.65[-8]$ & 6.008 & $1.87[-5]$ \\
$2 s 2 p\left({ }^{3} P\right) 7 f$ & -24.144227 & 4.35587 & $6.24[-8]$ & 7.008 & $2.15[-5]$ \\
$2 s 2 p\left({ }^{3} P\right) 8 f$ & -24.141843 & 4.42073 & $4.59[-8]$ & 8.008 & $2.36[-5]$ \\
$2 s 2 p\left({ }^{3} P\right) 9 f$ & -24.140208 & 4.46522 & $3.47[-8]$ & 9.008 & $2.53[-5]$ \\
$2 s 2 p\left({ }^{3} P\right) 10 f$ & -24.139038 & 4.49706 & $2.70[-8]$ & 10.007 & $2.71[-5]$ \\
$2 s 2 p\left({ }^{3} P\right)$ & -24.134046 & 4.63290 & & & \\
\hline \hline
\end{tabular}

states $\left(\bar{\Gamma} \sim 10^{-2}-10^{-3}\right.$ a.u. $)$, as well as the three intruder states $2 p^{2}\left({ }^{1} D\right) 3 d-5 d$. The latter are readily recognized from their positions: Their apparent effective principal quantum numbers $n^{*}$ with respect to the $2 p^{2}\left({ }^{3} P\right)$ threshold, extrapolated from the quantum defect of the $2 p^{2}\left({ }^{1} D\right) 6 d-9 d$ terms above threshold, are $n_{2 p^{2}\left({ }^{1} D\right) 3 d}^{* \text {, Guess }}=3.514, n_{2 p^{2}\left({ }^{1} D\right) 4 d}^{* \text {, uuess }}=5.591$, and $n_{2 p^{2}\left({ }^{1} D\right) 5 d}^{* \text { Guess }}=10.337$, in excellent agreement with the values of $3.539,5.595$, and 10.356, respectively, obtained from resonance fitting. Furthermore, all these three states exhibit very characteristic branching ratio that are clearly distinct from those of the two main series. While the $2 p^{2}\left({ }^{3} P\right) n s$ states seem to be quite insensitive to the presence of the intruder states, the reduced width of the $2 p^{2}\left({ }^{3} P\right) n d$ series exhibits a pronounced modulation that indicates a strong interaction with the $2 p^{2}\left({ }^{1} D\right) n d$ intruders. This hypothesis, which is reasonable already on the basis of the similarity between the dominant configurations of these states, is confirmed by the decay pattern of the higher terms in the $2 p^{2}\left({ }^{1} D\right) n d$ series in the following energy interval, which shows that the branching ratio to the $2 p^{2}\left({ }^{3} P\right) \varepsilon_{d}$ channel dominates $(\sim 65 \%)$, while that to the $2 p^{2}\left({ }^{3} P\right) \varepsilon_{s}$ channel is negligible $(1.5 \%)$.

The fourth and last interval, between the $2 p^{2}\left({ }^{1} D\right)$ and the $2 s 3 p\left({ }^{3} P\right)$ threshold, harbors, beyond the main $2 s 3 p\left({ }^{3} P\right) n p$ series, as many as 30 terms of the $2 s 3 p\left({ }^{1} P\right) n p$ series, whose limit is just $13 \mathrm{meV}$ above the $2 s 3 p\left({ }^{3} P\right)$ threshold. As a result, these two sequences give rise to alternating resonances, recognizable as a sequence of pairs of distinct peaks in the density of states (compare with the seventh panel from above in Fig. 3) up to large principal quantum numbers. The same interval features also the $2 s 3 d^{2}$ and the $2 s 3 d\left({ }^{3} D\right) 4 d$ intruder states, each affecting one term in both $2 s 3 p\left({ }^{1,3} P\right) n p$ series. In Fig. 3, the intruder states appear as the second and third green narrow peaks. The strong interaction of the $2 s 3 d^{2}$ with the $2 s 3 p\left({ }^{3} P\right) 5 p$, in particular, displaces the latter significantly and imparts to it a singularly large preference for the decay to the $2 p^{2}\left({ }^{1} D\right) \varepsilon_{d}$ channel.

\section{4. ${ }^{2} D^{e}$ autoionizing states}

The autoionizing states of the ${ }^{2} D^{e}$ manifold are reported in Tables XII, XIII, and XIV. Additional values are provided in the Supplemental Material [117].

The first energy interval, between the $2 s^{2}$ and the $2 s 2 p\left({ }^{3} \mathrm{P}\right)$ thresholds, has two main autoionizing series. The first term of the larger $2 s 2 p\left({ }^{3} P\right) n p$ series, with $2 s 2 p^{2}$ configuration, actually gives the largest contribution to the ground state in this symmetry, as already discussed in Sec. III C. A substantial energy shift with respect to the single-channel Rydberg states is observed also for the $2 s 2 p\left({ }^{3} P\right) 3 p$ and $2 s 2 p\left({ }^{3} P\right) 4 p$ states. The $2 s 2 p\left({ }^{3} P\right) n f$ series is much narrower, with a reduced width of the order of $10^{-5}$ a.u., which exhibits a pronounced energy dependence. To the best of our knowledge, the $2 s 2 p\left({ }^{3} P\right) 3 p{ }^{2} D^{e}$ resonance is the only other autoionizing state with symmetry pertinent to the present work, beyond the $2 s 2 p\left({ }^{3} P\right) 3 p{ }^{2} S^{e}$ already discussed in Sec. IIID 2, whose position has been claimed to have been determined experimentally [120]. In this case, the values reported in the literature, $E_{2 s 2 p\left({ }^{3} P\right) 3 p\left({ }^{2} D_{3 / 2}^{e}\right)}=$ $2.55775 \mathrm{eV}$ and $E_{2 s 2 p\left({ }^{3} P\right) 3 p\left({ }^{2} D_{5 / 2}^{e}\right)}=2.55807 \mathrm{eV}$, differ from the present theoretical value of $2.56964 \mathrm{eV}$ by $12 \mathrm{meV}$ 
TABLE XIII. Parameters of the ${ }^{2} D^{e}$ autoionizing states between the $2 s 2 p\left({ }^{3} P\right)$ and the $2 p^{2}\left({ }^{1} D\right)$ thresholds. The decay channels are, in order, $2 s^{2} \varepsilon_{d}, 2 s 2 p\left({ }^{3} P\right) \varepsilon_{p}, 2 s 2 p\left({ }^{3} P\right) \varepsilon_{f}, 2 s 2 p\left({ }^{1} P\right) \varepsilon_{p}, 2 s 2 p\left({ }^{1} P\right) \varepsilon_{f}$, and $2 p^{2}\left({ }^{3} P\right) \varepsilon_{d}$.

\begin{tabular}{|c|c|c|c|c|c|c|c|c|c|c|c|}
\hline \multirow[b]{2}{*}{ State } & \multirow[b]{2}{*}{$E$ (a.u.) } & \multirow[b]{2}{*}{$E(\mathrm{eV})$} & \multirow[b]{2}{*}{$\Gamma$ (a.u.) } & \multirow[b]{2}{*}{$n^{*}$} & \multirow[b]{2}{*}{$\bar{\Gamma}$ (a.u.) } & \multicolumn{6}{|c|}{ Branching ratios $(\%)$} \\
\hline & & & & & & $\mathrm{ch}_{1}$ & $\mathrm{ch}_{2}$ & $\mathrm{ch}_{3}$ & $\mathrm{ch}_{4}$ & $\mathrm{ch}_{5}$ & $\mathrm{ch}_{6}$ \\
\hline $2 s 2 p\left({ }^{1} P\right) 4 f$ & -23.989463 & 8.56699 & $1.28[-7]$ & 4.994 & $1.59[-5]$ & 57.9 & 24.1 & 18.0 & & & \\
\hline $2 s 2 p\left({ }^{1} P\right) 5 f$ & -23.983337 & 8.73368 & $9.63[-8]$ & 5.993 & $2.07[-5]$ & 55.9 & 26.6 & 17.5 & & & \\
\hline $2 s 2 p\left({ }^{1} P\right) 6 f$ & -23.979642 & 8.83422 & $7.11[-8]$ & 6.993 & $2.43[-5]$ & 54.3 & 28.7 & 17.0 & & & \\
\hline $2 s 2 p\left({ }^{1} P\right) 7 f$ & -23.977244 & 8.89947 & $5.29[-8]$ & 7.992 & $2.70[-5]$ & 53.1 & 30.4 & 16.5 & & & \\
\hline $2 s 2 p\left({ }^{1} P\right) 8 f$ & -23.975601 & 8.94420 & $4.00[-8]$ & 8.992 & $2.91[-5]$ & 52.1 & 31.8 & 16.1 & & & \\
\hline $2 s 2 p\left({ }^{1} P\right) 9 f$ & -23.974425 & 8.97619 & $3.08[-8]$ & 9.992 & $3.07[-5]$ & 51.2 & 33.0 & 15.8 & & & \\
\hline $2 s 2 p\left({ }^{1} P\right) 10 f$ & -23.973555 & 8.99985 & $2.42[-8]$ & 10.991 & $3.21[-5]$ & 50.6 & 34.0 & 15.5 & & & \\
\hline $2 s 2 p\left({ }^{1} P\right) 3 p$ & -24.057946 & 6.70357 & $1.98[-3]$ & 2.377 & $2.65[-2]$ & 10.3 & 89.5 & 0.3 & & & \\
\hline $2 s 2 p\left({ }^{1} P\right) 4 p$ & -24.012632 & 7.93657 & $7.60[-4]$ & 3.401 & $2.99[-2]$ & 10.3 & 89.3 & 0.3 & & & \\
\hline $2 s 2 p\left({ }^{1} P\right) 5 p$ & -23.995149 & 8.41229 & $3.89[-4]$ & 4.408 & $3.33[-2]$ & 10.0 & 89.7 & 0.4 & & & \\
\hline $2 s 2 p\left({ }^{1} P\right) 6 p$ & -23.986500 & 8.64764 & $2.30[-4]$ & 5.410 & $3.64[-2]$ & 9.6 & 90.0 & 0.4 & & & \\
\hline $2 s 2 p\left({ }^{1} P\right) 7 p$ & -23.981583 & 8.78141 & $1.49[-4]$ & 6.411 & $3.91[-2]$ & 9.3 & 90.3 & 0.4 & & & \\
\hline $2 s 2 p\left({ }^{1} P\right) 8 p$ & -23.978522 & 8.86472 & $1.02[-4]$ & 7.410 & $4.14[-2]$ & 9.1 & 90.5 & 0.4 & & & \\
\hline $2 s 2 p\left({ }^{1} P\right) 9 p$ & -23.976486 & 8.92011 & $7.30[-5]$ & 8.410 & $4.34[-2]$ & 8.9 & 90.7 & 0.4 & & & \\
\hline $2 s 2 p\left({ }^{1} P\right) 10 p$ & -23.975064 & 8.95881 & $5.42[-5]$ & 9.410 & $4.51[-2]$ & 8.8 & 90.8 & 0.4 & & & \\
\hline $2 s 2 p\left({ }^{1} P\right) 11 p$ & -23.974031 & 8.98690 & $4.13[-5]$ & 10.409 & $4.66[-2]$ & 8.7 & 90.9 & 0.4 & & & \\
\hline $2 s 2 p\left({ }^{1} P\right)$ & -23.969417 & 9.11247 & & & & & & & & & \\
\hline $2 p^{2}\left({ }^{3} P\right) 3 d$ & -23.906838 & 10.81524 & $8.58[-4]$ & 3.046 & $2.43[-2]$ & 0.5 & 9.2 & 90.0 & 0.2 & 0.0 & \\
\hline $2 p^{2}\left({ }^{3} P\right) 4 d$ & -23.883526 & 11.44954 & $4.73[-4]$ & 4.045 & $3.13[-2]$ & 0.6 & 12.9 & 86.0 & 0.5 & 0.0 & \\
\hline $2 p^{2}\left({ }^{3} P\right) 5 d$ & -23.872623 & 11.74620 & $2.57[-4]$ & 5.043 & $3.29[-2]$ & 0.6 & 10.1 & 89.2 & 0.2 & 0.0 & \\
\hline $2 p^{2}\left({ }^{3} P\right) 6 d$ & -23.866662 & 11.90842 & $1.85[-4]$ & 6.042 & $4.07[-2]$ & 0.5 & 17.6 & 78.7 & 3.1 & 0.0 & \\
\hline $2 p^{2}\left({ }^{3} P\right) 7 d$ & -23.863046 & 12.00680 & $1.02[-4]$ & 7.042 & $3.55[-2]$ & 0.6 & 12.4 & 86.7 & 0.3 & 0.0 & \\
\hline $2 p^{2}\left({ }^{3} P\right) 8 d$ & -23.860696 & 12.07075 & $6.85[-5]$ & 8.042 & $3.56[-2]$ & 0.6 & 11.3 & 87.9 & 0.2 & 0.0 & \\
\hline $2 p^{2}\left({ }^{3} P\right) 9 d$ & -23.859081 & 12.11469 & $4.86[-5]$ & 9.041 & $3.59[-2]$ & 0.6 & 10.3 & 88.9 & 0.2 & 0.0 & \\
\hline $2 p^{2}\left({ }^{3} P\right) 10 d$ & -23.857914 & 12.14644 & $2.59[-5]$ & 10.051 & $2.63[-2]$ & 1.5 & 4.9 & 57.0 & 17.2 & 19.5 & \\
\hline $2 p^{2}\left({ }^{3} P\right) 11 d$ & -23.857067 & 12.16950 & $2.46[-5]$ & 11.040 & $3.32[-2]$ & 0.4 & 3.0 & 95.8 & 0.8 & 0.1 & \\
\hline $2 p^{2}\left({ }^{3} P\right) 12 d$ & -23.856414 & 12.18727 & $2.42[-5]$ & 12.040 & $4.22[-2]$ & 0.5 & 17.9 & 78.4 & 3.0 & 0.1 & \\
\hline $2 p^{2}\left({ }^{1} D\right) 3 d$ & -23.894657 & 11.14668 & $2.47[-4]$ & 3.463 & $1.03[-2]$ & 4.6 & 6.7 & 7.7 & 31.6 & 49.3 & \\
\hline $2 p^{2}\left({ }^{1} D\right) 4 d$ & -23.869540 & 11.83011 & $1.14[-4]$ & 5.492 & $1.89[-2]$ & 5.0 & 6.1 & 9.7 & 36.7 & 42.5 & \\
\hline $2 p^{2}\left({ }^{1} D\right) 5 d$ & -23.857961 & 12.14517 & $6.84[-5]$ & 10.004 & $6.85[-2]$ & 4.2 & 7.4 & 33.9 & 27.4 & 27.2 & \\
\hline $2 p^{2}\left({ }^{1} D\right) 3 s$ & -23.951380 & 9.60324 & $7.20[-3]$ & 2.254 & $8.24[-2]$ & 0.8 & 66.9 & 0.4 & 31.9 & 0.0 & \\
\hline $2 p^{2}\left({ }^{1} D\right) 4 s$ & -23.889107 & 11.29769 & $2.06[-3]$ & 3.719 & $1.06[-1]$ & 0.5 & 65.4 & 0.8 & 33.2 & 0.1 & \\
\hline $2 p^{2}\left({ }^{1} D\right) 5 s$ & -23.867005 & 11.89909 & $8.43[-4]$ & 5.968 & $1.79[-1]$ & 0.3 & 60.4 & 6.7 & 32.5 & 0.1 & \\
\hline $2 p^{2}\left({ }^{1} D\right) 6 s$ & -23.856608 & 12.18198 & $4.48[-4]$ & 11.715 & $7.20[-1]$ & 0.3 & 65.4 & 1.5 & 32.5 & 0.1 & \\
\hline $2 p^{2}\left({ }^{1} D\right) 5 g$ & -23.857435 & 12.15949 & $1.66[-8]$ & 10.576 & $1.96[-5]$ & & & & & & \\
\hline $2 p^{2}\left({ }^{3} P\right)$ & -23.852965 & 12.28111 & & & & & & & & & \\
\hline $2 p^{2}\left({ }^{1} D\right) 6 d$ & -23.851679 & 12.31610 & $3.58[-5]$ & 5.950 & $7.54[-3]$ & 5.1 & 4.5 & 10.3 & 38.9 & 36.8 & 4.4 \\
\hline $2 p^{2}\left({ }^{1} D\right) 7 d$ & -23.847910 & 12.41867 & $2.25[-5]$ & 6.949 & $7.56[-3]$ & 5.1 & 4.3 & 10.5 & 39.5 & 35.9 & 4.6 \\
\hline $2 p^{2}\left({ }^{1} D\right) 8 d$ & -23.845470 & 12.48506 & $1.51[-5]$ & 7.949 & $7.56[-3]$ & 5.1 & 4.2 & 10.7 & 39.9 & 35.3 & 4.8 \\
\hline $2 p^{2}\left({ }^{1} D\right) 9 d$ & -23.843800 & 12.53048 & $1.05[-5]$ & 8.948 & $7.56[-3]$ & 5.1 & 4.1 & 10.9 & 40.1 & 34.9 & 4.9 \\
\hline $2 p^{2}\left({ }^{1} D\right) 10 d$ & -23.842608 & 12.56292 & $7.67[-6]$ & 9.948 & $7.55[-3]$ & 5.1 & 4.0 & 11.0 & 40.3 & 34.6 & 5.0 \\
\hline $2 p^{2}\left({ }^{1} D\right) 11 d$ & -23.841728 & 12.58688 & $5.74[-6]$ & 10.948 & $7.54[-3]$ & 5.1 & 4.0 & 11.0 & 40.4 & 34.4 & 5.0 \\
\hline $2 p^{2}\left({ }^{1} D\right) 7 s$ & -23.850890 & 12.33756 & $2.64[-4]$ & 6.123 & $6.07[-2]$ & 0.3 & 65.5 & 0.6 & 32.2 & 0.1 & 1.2 \\
\hline $2 p^{2}\left({ }^{1} D\right) 8 s$ & -23.847407 & 12.43233 & $1.67[-4]$ & 7.124 & $6.05[-2]$ & 0.3 & 65.7 & 0.6 & 32.0 & 0.2 & 1.3 \\
\hline $2 p^{2}\left({ }^{1} D\right) 9 s$ & -23.845131 & 12.49428 & $1.13[-4]$ & 8.125 & $6.04[-2]$ & 0.3 & 65.9 & 0.6 & 31.8 & 0.2 & 1.3 \\
\hline $2 p^{2}\left({ }^{1} D\right) 10 s$ & -23.843561 & 12.53700 & $7.95[-5]$ & 9.125 & $6.04[-2]$ & 0.3 & 66.0 & 0.6 & 31.7 & 0.2 & 1.3 \\
\hline $2 p^{2}\left({ }^{1} D\right) 11 s$ & -23.842433 & 12.56768 & $5.82[-5]$ & 10.125 & $6.04[-2]$ & 0.3 & 66.1 & 0.6 & 31.6 & 0.2 & 1.3 \\
\hline $2 p^{2}\left({ }^{1} D\right)$ & -23.837556 & 12.70039 & & & & & & & & & \\
\hline
\end{tabular}


TABLE XIV. Parameters of the ${ }^{2} D^{e}$ autoionizing states between the $2 p^{2}\left({ }^{1} D\right)$ and the $2 p^{2}\left({ }^{1} S\right)$ thresholds. The decay channels are, in order, $2 s^{2} \varepsilon_{d}, 2 s 2 p\left({ }^{3} P\right) \varepsilon_{p}, 2 s 2 p\left({ }^{3} P\right) \varepsilon_{f}, 2 s 2 p\left({ }^{1} P\right) \varepsilon_{p}, 2 s 2 p\left({ }^{1} P\right) \varepsilon_{f}, 2 p^{2}\left({ }^{3} P\right) \varepsilon_{d}, 2 p^{2}\left({ }^{1} D\right) \varepsilon_{s}, 2 p^{2}\left({ }^{1} D\right) \varepsilon_{d}$, and $2 p^{2}\left({ }^{1} D\right) \varepsilon_{g}$.

\begin{tabular}{|c|c|c|c|c|c|c|c|c|c|c|c|c|c|c|}
\hline \multirow[b]{2}{*}{ Main configuration } & \multirow[b]{2}{*}{$E$ (a.u.) } & \multirow[b]{2}{*}{$E(\mathrm{eV})$} & \multirow[b]{2}{*}{$\Gamma$ (a.u.) } & \multirow[b]{2}{*}{$n^{*}$} & \multirow[b]{2}{*}{$\bar{\Gamma}$ (a.u.) } & \multicolumn{9}{|c|}{ Branching ratios $(\%)$} \\
\hline & & & & & & $\mathrm{ch}_{1}$ & $\mathrm{ch}_{2}$ & $\mathrm{ch}_{3}$ & $\mathrm{ch}_{4}$ & $\mathrm{ch}_{5}$ & $\mathrm{ch}_{6}$ & $\mathrm{ch}_{7}$ & $\mathrm{ch}_{8}$ & $\mathrm{ch}_{9}$ \\
\hline $2 p^{2}\left({ }^{1} S\right) 3 d$ & -23.788102 & 14.04605 & $3.76[-3]$ & 2.762 & $7.91[-2]$ & 5.2 & 4.9 & 0.4 & 0.6 & 73.5 & 4.9 & 6.1 & 1.0 & 3.4 \\
\hline $2 p^{2}\left({ }^{1} S\right) 4 d$ & -23.756360 & 14.90973 & $1.29[-3]$ & 3.846 & $7.33[-2]$ & 2.5 & 9.8 & 0.9 & 3.3 & 72.0 & 7.3 & 0.7 & 0.9 & 2.5 \\
\hline $2 p^{2}\left({ }^{1} S\right) 5 d$ & -23.742743 & 15.28024 & $1.35[-3]$ & 4.977 & $1.66[-1]$ & 0.2 & 2.5 & 1.6 & 6.3 & 58.9 & 11.9 & 0.3 & 9.4 & 8.9 \\
\hline $2 p^{2}\left({ }^{1} S\right) 6 d$ & -23.737126 & 15.43310 & $8.18[-4]$ & 5.859 & $1.64[-1]$ & 3.1 & 1.9 & 0.8 & 6.5 & 69.1 & 9.8 & 0.4 & 3.2 & 5.3 \\
\hline $2 p^{2}\left({ }^{1} S\right) 7 d$ & -23.733301 & 15.53717 & $4.49[-4]$ & 6.823 & $1.43[-1]$ & 4.0 & 2.5 & 0.7 & 4.8 & 71.2 & 9.6 & 0.1 & 2.7 & 4.4 \\
\hline $2 p^{2}\left({ }^{1} S\right) 8 d$ & -23.730784 & 15.60565 & $2.82[-4]$ & 7.797 & $1.34[-1]$ & 5.8 & 3.3 & 0.6 & 5.6 & 70.1 & 8.7 & 0.0 & 2.2 & 3.6 \\
\hline $2 p^{2}\left({ }^{1} S\right) 9 d$ & -23.729062 & 15.65252 & $2.00[-4]$ & 8.769 & $1.35[-1]$ & 9.2 & 5.1 & 0.8 & 10.2 & 62.7 & 6.9 & 0.7 & 1.7 & 2.6 \\
\hline $2 p^{2}\left({ }^{1} S\right) 10 d$ & -23.727799 & 15.68688 & $1.20[-4]$ & 9.769 & $1.12[-1]$ & 7.0 & 4.2 & 0.6 & 4.3 & 70.8 & 8.3 & 0.1 & 1.9 & 2.8 \\
\hline $2 s 3 s\left({ }^{3} S\right) 3 d$ & -23.801461 & 13.68254 & $4.56[-3]$ & 2.517 & $7.28[-2]$ & 10.0 & 33.2 & 2.2 & 34.3 & 6.8 & 0.0 & 9.8 & 3.7 & 0.1 \\
\hline $2 s 3 s\left({ }^{3} S\right) 4 d$ & -23.750545 & 15.06796 & $1.09[-3]$ & 4.227 & $8.26[-2]$ & 0.1 & 0.3 & 1.0 & 20.2 & 37.4 & 10.6 & 14.1 & 5.0 & 11.3 \\
\hline $2 s 3 s\left({ }^{3} S\right) 5 d$ & -23.735990 & 15.46401 & $2.02[-4]$ & 6.102 & $4.59[-2]$ & 11.4 & 0.4 & 14.2 & 28.6 & 0.1 & 1.0 & 31.3 & 12.7 & 0.4 \\
\hline $2 s 3 s\left({ }^{3} S\right) 6 d$ & -23.728754 & 15.66089 & $9.78[-5]$ & 8.984 & $7.10[-2]$ & 9.6 & 5.9 & 11.4 & 33.1 & 2.9 & 1.4 & 27.1 & 7.9 & 0.5 \\
\hline $2 s 3 p^{2}$ & -23.764984 & 14.67508 & $1.17[-3]$ & 3.433 & $4.72[-2]$ & 13.5 & 62.2 & 1.1 & 1.8 & 1.7 & 4.6 & 9.1 & 0.8 & 5.3 \\
\hline $2 s 3 s\left({ }^{1} S\right) 4 d$ & -23.745677 & 15.20041 & $5.59[-4]$ & 4.651 & $5.62[-2]$ & 16.0 & 14.0 & 10.6 & 0.4 & 23.0 & 1.4 & 6.7 & 27.1 & 0.7 \\
\hline $2 p^{2}\left({ }^{1} S\right)$ & -23.722560 & 15.82943 & & & & & & & & & & & & \\
\hline
\end{tabular}

(compare with Table XII), which is, in principle, a satisfactory agreement. Indeed, a 12-meV difference is compatible with the accuracy expected for the present calculation, and it is only a fraction of the resonance width, which is of the order of 50 $\mathrm{meV}$, and which should be unambiguously visible as an almost window resonance in the transition from the ground state (see Fig. 7). That said, the experiment reports two different values for the position of the $3 / 2$ and $5 / 2$ components of the autoionizing multiplet, supposedly separated by a mere 0.32 meV, i.e., less than $1 \%$ of the resonance width. This apparently contradictory circumstance, together with the lack of any details on how the transitions are extracted from the measured spectrum, which is not available, or the assignments made, calls those experimental results into question and suggests that further experimental investigation is needed.

In the second interval, between the $2 s 2 p\left({ }^{3} P\right)$ and the $2 s 2 p\left({ }^{1} P\right)$ thresholds, there are again two main series, the

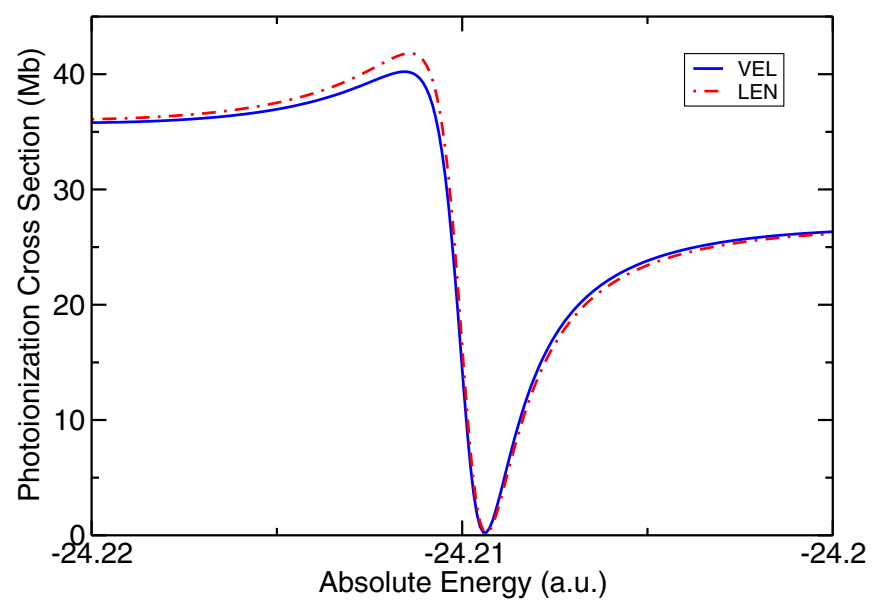

FIG. 7. Photoionization cross section of the boron atom from the $2 s^{2} 2 p{ }^{2} P^{o}$ ground state to the $2 s^{2} \varepsilon_{d}$ ionization continuum, in the proximity of the $2 s 2 p\left({ }^{3} P\right) 3 p\left({ }^{2} D\right)$ autoionizing state. See Fig. 4 and text for further details. narrow $2 s 2 p\left({ }^{1} P\right) n f$ series, and a much broader $2 s 2 p\left({ }^{1} P\right) n p$ series, with a very pronounced preferential decay to the $2 s 2 p\left({ }^{3} P\right) \varepsilon_{p}$ channel $(\sim 90 \%)$. In this case, both these seemingly regular series actually exhibit a steady increase of their reduced width, possibly as a result of the influence of the $2 p^{2}\left({ }^{1} D\right) 3 s$, which is located slightly above the $2 s 2 p\left({ }^{1} P\right)$ threshold.

The third interval, between the $2 s 2 p\left({ }^{1} P\right)$ and the $2 p^{2}\left({ }^{3} P\right)$ thresholds, has a very rich structure due to the presence, just $15 \mathrm{meV}$ above its upper limit, of the $2 p^{2}\left({ }^{1} D\right)$ threshold, which binds several Rydberg satellites, with $s, d$, and $g$ angular momentum and that appear here as intruder states. The distribution of the main series and of the intruder states is clearly seen in the second panel from the bottom in Fig. 3. The regular series is given by the light-violet peaks, which indicates

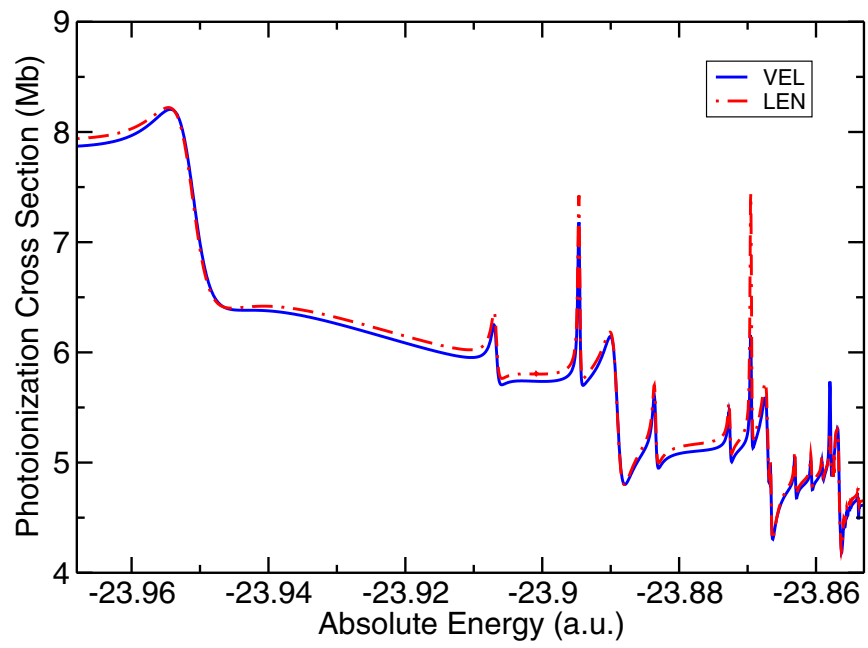

FIG. 8. Total photoionization cross section of the boron atom from the $2 s^{2} 2 p$ ground state to the ${ }^{2} D^{e}$ ionization continuum, between the $2 s 2 p\left({ }^{1} P\right)$ and the $2 p^{2}\left({ }^{3} P\right)$ thresholds. See Fig. 4 and text for further details. 
a preferential decay by emission of an electron with angular momentum larger than 2 . Indeed, the main series $2 p^{2}\left({ }^{3} P\right) n d$ decays preponderantly to the $2 s 2 p\left({ }^{3} P\right) \varepsilon_{f}$ channel. The lightblue peaks correspond to the $2 p^{2}\left({ }^{1} D\right) 3 d-5 d$ intruder states, the last of which interacts strongly with the $2 p^{2}\left({ }^{3} P\right) 10 d$ term of the main series. The $2 p^{2}\left({ }^{1} D\right) 3 s-6 s$ intruder states give rise to the broadest resonances, which decay preferentially to the $2 s 2 p\left({ }^{3} P\right) \varepsilon_{p}$ channel. The intruder $2 p^{2}\left({ }^{1} D\right) 5 g$ gives rise to a vanishingly narrow resonance, with $\Gamma=1.66 \times 10^{-8}$ a.u. Beyond $n^{*}=12$, where no further intruder states can be found, the parameters of the main $2 p^{2}\left({ }^{3} P\right) n d$ series steadily approach their asymptotic values.

The many intruder states in this region give also rise to recognizable resonant features in the total photoionization cross section from the ground state (see Fig. 8), superposed to those of the denser main series.

In the narrow energy interval between the $2 p^{2}\left({ }^{3} P\right)$ and the $2 p^{2}\left({ }^{1} D\right)$ thresholds there are, in principle, three regular main series. The terms of the narrowest $2 p^{2}\left({ }^{1} D\right) n g$ series, however, are virtually bound and have not been resolved here. The two remaining $2 p^{2}\left({ }^{1} D\right) n s$ and $2 p^{2}\left({ }^{1} D\right) n d$ series have very stable reduced parameters. For both series, the branching ratio to the newly open $2 p^{2}\left({ }^{3} P\right) \varepsilon_{d}$ channel is very small, and the branching ratio to the other channels closely reproduces that of the corresponding intruder states examined in the previous energy interval.

Finally, as in the case of the other two natural symmetries, the fifth and last interval, between the $2 p^{2}\left({ }^{1} D\right)$ and the $2 p^{2}\left({ }^{1} S\right)$ thresholds, features one main series and numerous intruder states from the nearby $2 s 3 s\left({ }^{3,1} S\right)$ and $2 s 3 p\left({ }^{3} P\right)$ thresholds. The parameter of the resonances in this energy region are listed in Table XIV, together with their tentative assignment. The density of states corresponding to this interval, shown in the bottom panel of Fig. 3, conveys the idea that, while for $n^{*} \geqslant 5$ there is a reasonably clear-cut distinction between the $2 p^{2}\left({ }^{1} S\right) n d$ main series and the intruder states, at smaller energies the terms of the main series are strongly mixed with the four $2 s 3 s\left({ }^{3} S\right) 3 d, 2 s 3 s\left({ }^{3} S\right) 4 d, 2 s 3 s\left({ }^{1} S\right) 3 d$, and $2 s 3 p^{2}$ intruder states.

\section{CONCLUSIONS}

We have presented a new implementation of the $B$-spline $K$-matrix method, based on the close-coupling expansion with a localized channel to complement short-range correlation, for three-active electron atoms with a polarizable core, in which the core-valence interaction potential comprises both a one-body and a two-body dipolar component. The method is designed to predict, at a moderate computational cost and with competitive and uniform precision, the properties of the single-ionization continuum of polyelectronic atoms, and it has been applied to compute a large number of bound and autoionizing doublet states of the neutral boron atom in the ${ }^{2} S^{e},{ }^{2} P^{e, o}$, and ${ }^{2} D^{e}$ symmetries, for which little or no data were previously available, thus filling an important gap in the literature. The present theoretical data are in very good agreement with the few validated experimental and theoretical records that are available for comparison. The extensive theoretical analysis conducted in this work permitted us to highlight several rearrangement processes driven by the electronic correlation between three similarly excited electrons. Finally, the $\mathrm{CC}+\mathrm{LC}$ bases examined in this work constitute a suitable space in which to conduct time-resolved studies of the correlated dynamics of three electrons triggered by subfemtosecond extreme-ultraviolet pulses. Therefore, this work, which demonstrates the possibility of building accurate scattering states within those same spaces, opens the way to the "exact" asymptotic analysis of correlated three-electron wave packets in the single-ionization continuum of three-electron systems, in a way similar to the one that has already been successfully employed for the helium atom [83-86,88].

\section{ACKNOWLEDGMENTS}

L.A. acknowledges support from the European Research Council under the European Union's Seventh Framework Programme (FP7/2007-2013)/ERC grant XCHEM 290853, the MINECO project no. FIS2013-42002-R, the ERA-Chemistry Project PIM2010EEC-00751, the European grant MC-ITN CORINF and the European COST Action XLIC CM1204.

\section{APPENDIX : MATRIX ELEMENTS BETWEEN THREE-ELECTRON CONFIGURATIONS}

In this Appendix we provide the expressions for the matrix elements of one- and two-body spin-free operators, such as the Hamiltonian and the dipole transition operators, between products of three angularly coupled electrons, $|123\rangle$, built from nonorthogonal radial orbitals,

$$
\left\langle\left\{\vec{r}_{i}\right\} \mid 123\right\rangle=\left[\left[\phi_{n_{1} \ell_{1}}\left(\vec{r}_{1}\right) \otimes \phi_{n_{2} \ell_{2}}\left(\vec{r}_{2}\right)\right]_{\ell_{12}} \otimes \phi_{n_{3} \ell_{3}}\left(\vec{r}_{3}\right)\right]_{L M} .
$$

For bra states, we use primed indexes. As shown in Sec. II B, one needs to evaluate matrix elements of operators of the form $n_{\gamma}^{-1} O \hat{\rho}_{S_{a} S_{b}}^{\bar{\gamma}_{s}}$, where $\bar{\gamma}_{s}$ identifies the total spin, whereas $S_{a}$ and $S_{b}$ indicate the intermediate spin coupling, between the first two electrons, in the bra and ket states, respectively. In the case of one-body operators, such as the kinetic energy or the dipole, we can write $n_{\gamma}^{-1} O \hat{\rho}_{S_{a} S_{b}}^{\bar{\gamma}_{s}}=\frac{1}{6} \sum_{i \sigma} D_{S_{a} S_{b}}^{\bar{\gamma} *}(\sigma) O(i) \sigma$, whereas for two-body operators we can write $n_{\gamma}^{-1} G \hat{\rho}_{S_{a} s_{b}}^{\bar{s}_{s}}=$ $\frac{1}{6} \sum_{k \sigma} D_{S_{a} S_{b}}^{\bar{\gamma} *}(\sigma) g(i, j) \sigma$, where it is assumed that in the last expression $i j k$ are cyclic permutations of 123 . The full matrix elements between antisymmetrized spin and angularly coupled three-electron states, therefore, are simple linear combinations of matrix elements of the form $\left\langle 1^{\prime} 2^{\prime} 3^{\prime}\left\|o_{T}(i) \sigma\right\| 123\right\rangle$, for onebody tensor operator of rank $T$, and $\left\langle 1^{\prime} 2^{\prime} 3^{\prime} \mid g_{(i, j) \sigma} \sigma 123\right\rangle$, for two-body operators. In the latter case, in particular, we are interested in the interelectronic repulsion $r_{12}^{-1}=\sum_{l} g_{l}(1,2)$, with $g_{l}(1,2)=r_{<}^{l} / r_{>}^{l+1} P_{l}\left(\hat{r}_{1} \cdot \hat{r}_{2}\right)$. These matrix elements can be written as the product of overlap integrals, radial transition integrals, and angular coefficients $\mathcal{F}_{i \sigma}$ and $\mathcal{G}_{k \sigma}$,

$$
\begin{aligned}
& \left\langle 1^{\prime} 2^{\prime} 3^{\prime}\left\|o_{T}(i) \sigma\right\| 123\right\rangle=\mathcal{F}_{i \sigma} \prod_{\ell_{12}^{\prime} \ell_{12} L L^{\prime}}\left\langle i^{\prime}\left\|o_{T}\right\| \bar{\sigma}_{i}\right\rangle \prod_{j \neq i}\left\langle j^{\prime} \mid \bar{\sigma}_{j}\right\rangle, \\
& \left\langle 1^{\prime} 2^{\prime} 3^{\prime}\left|g_{l}(i, j) \sigma\right| 123\right\rangle=\mathcal{G}_{k \sigma} \prod_{\ell_{12}^{\prime} \ell_{12}}\left\{i^{\prime} j^{\prime} \mid \bar{\sigma}_{i} \bar{\sigma}_{j}\right\}_{l}\left\langle k^{\prime} \mid \bar{\sigma}_{k}\right\rangle, \\
& \text { with } \prod_{a b \cdots}=\sqrt{(2 a+1)(2 b+1) \cdots},\left\langle i^{\prime} \mid j\right\rangle=\delta_{\ell_{i}^{\prime} \ell_{j}}\left\langle\phi_{i}^{\prime} \mid \phi_{j}\right\rangle,\left\{1^{\prime} 2^{\prime} \mid 12\right\}_{l} \\
& =(-)^{l} \prod_{\ell_{1} \ell_{2}} C_{l 0, \ell_{1} 0}^{\ell_{1}^{\prime} 0} C_{l 0, \ell_{2} 0}^{\ell_{2}^{\prime} 0}\left\langle\phi_{1}^{\prime} \phi_{2}^{\prime}\left|\frac{r_{l}^{l}}{r_{>}^{l+1}}\right| \phi_{1} \phi_{2}\right\rangle \text {, and } \sigma \bar{\sigma}=1 \text {. The }
\end{aligned}
$$


TABLE XV. Coefficients $\mathcal{F}_{i \sigma}$ and $\mathcal{G}_{k \sigma}$, for the matrix elements of the one-body tensorial operator $\boldsymbol{o}_{T}(i) \sigma$ and the two-body multipolar scalar operator $g_{l}(i, j) \sigma$, respectively, between angularly coupled three-electron configurations. Here, the permutation $\sigma=(123)$ is defined such that $\sigma f\left(x_{1}, x_{2}, x_{3}\right) \equiv f\left(x_{\sigma_{1}}, x_{\sigma_{2}}, x_{\sigma_{3}}\right)=f\left(x_{3}, x_{1}, x_{2}\right)$, and hence $\sigma \phi_{1} \phi_{2} \phi_{3}=\phi_{\bar{\sigma}_{1}} \phi_{\bar{\sigma}_{2}} \phi_{\bar{\sigma}_{3}}=\phi_{2} \phi_{3} \phi_{1}$. The naturality $v$ is defined so that $v=1(v=-1)$ for natural (unnatural) states and operators. For all other angular symbols we follow the conventions in [121].

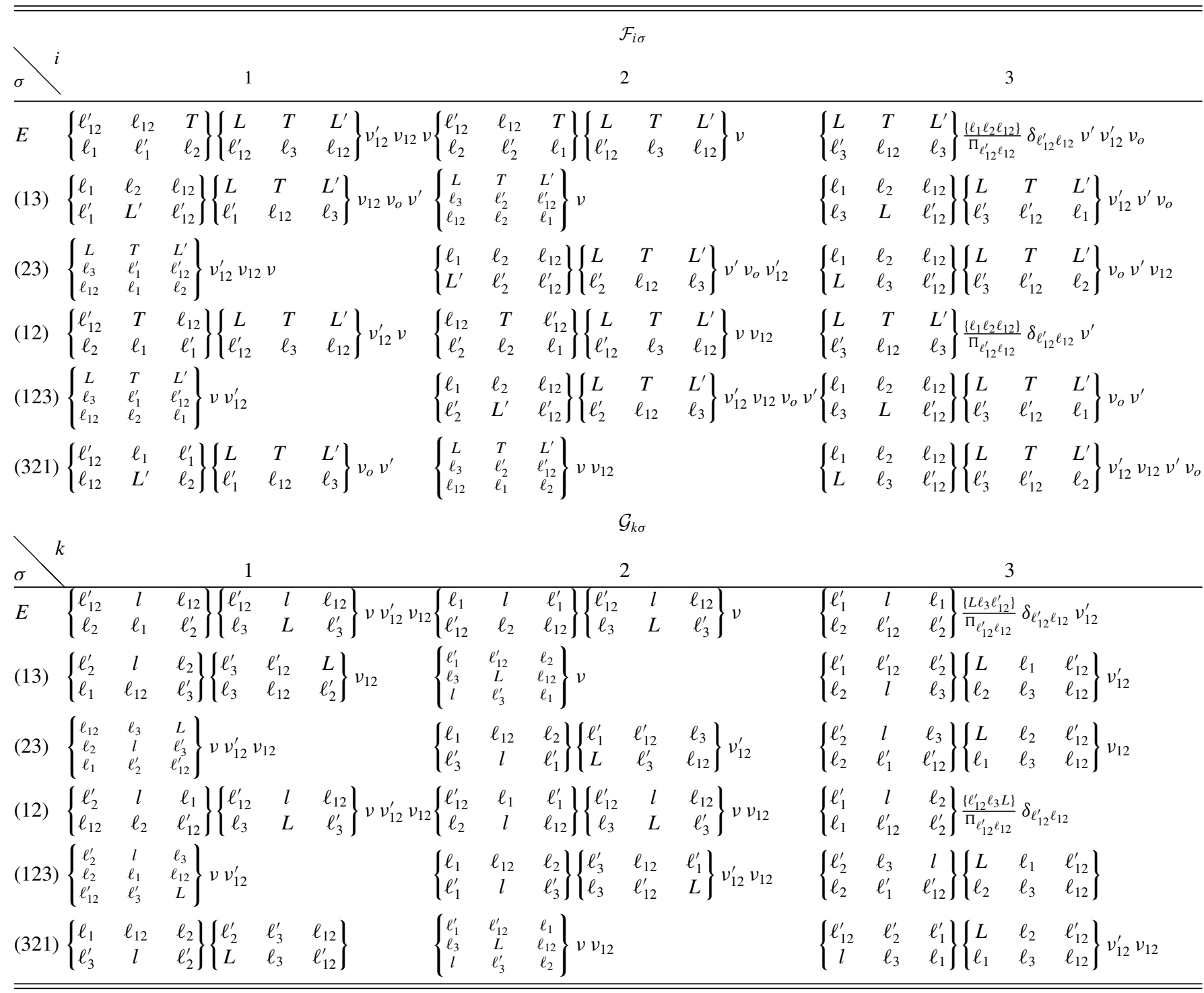

angular coefficients $\mathcal{F}_{i \sigma}$ and $\mathcal{G}_{k \sigma}$ can be computed with standard techniques of angular algebra [121]. Their expressions in terms of $3 n j$ symbols, as a function of bra and ket angular momentum quantum numbers, are listed in Table XV.
[1] T. S. Perry, S. J. Davidson, F. J. D. Serduke, D. R. Bach, C. C. Smith, J. M. Foster, R. J. Doyas, R. A. Ward, C. A. Iglesias, F. J. Rogers, J. Abdallah, R. E. Stewart, J. D. Kilkenny, and R. W. Lee, Phys. Rev. Lett. 67, 3784 (1991).

[2] U. Feldman, P. Mandelbaum, J. F. Seely, G. A. Doschek, and H. Gursky, Astrophys. J. Suppl. Ser. 81, 387 (1992).

[3] J. Dubau and S. Volonte, Rep. Prog. Phys. 43, 199 (1980).

[4] J. M. Miller, J. Raymond, C. S. Reynolds, A. C. Fabian, T. R. Kallman, and J. Homan, Astrophys. J 680, 1359 (2008).

[5] T. Yaqoob, I. M. George, K. Nandra, T. J. Turner, P. J. Serlemitsos, and R. F. Mushotzky, Astrophys. J 546, 759 (2001).
[6] K. R. Sembach and B. D. Savage, Astrophys. J. Suppl. Ser. 83, 147 (1992).

[7] J. S. Bolton and M. G. Haehnelt, Mon. Not. R. Acad. Sci. 382, 325 (2007).

[8] M. Donahue and J. M. Shull, Astrophys. J 383, 511 (1991).

[9] J. S. Kaastra and R. Mewe, Astron. Astrophys. Suppl. Ser. 97, 443 (1993).

[10] V. Schmidt, Rep. Prog. Phys. 55, 1483 (1992).

[11] B. Sonntag and P. Zimmermann, Rep. Prog. Phys. 55, 911 (1992).

[12] D. H. Bilderback, P. Elleaume, and E. Weckert, J. Phys. B: At. Mol. Opt. Phys. 38, S773 (2005). 
[13] W. F. Chan, G. Cooper, and C. E. Brion, Phys. Rev. A 44, 186 (1991).

[14] M. Domke, G. Remmers, and G. Kaindl, Phys. Rev. Lett. 69, 1171 (1992).

[15] M. Domke, K. Schulz, G. Remmers, G. Kaindl, and D. Wintgen, Phys. Rev. A 53, 1424 (1996).

[16] N. de Oliveira, M. Roudjane, D. Joyeux, D. Phalippou, J.-C. Rodier, and L. Nahon, Nature Photonics 5, 149 (2011).

[17] A. T. J. B. Eppink and D. H. Parker, Rev. Sci. Instrum. 68, 3477 (1997).

[18] J. Ullrich, R. Moshammer, A. Dorn, R. Dörner, L. P. H. Schmidt, and H. Schmidt-Böcking, Rep. Prog. Phys. 66, 1463 (2003).

[19] F. J. Wuilleumier and M. Meyer, J. Phys. B: At. Mol. Opt. Phys. 39, R425 (2006).

[20] P. O'Keeffe, E. V. Gryzlova, D. Cubaynes, G. A. Garcia, L. Nahon, A. N. Grum-Grzhimailo, and M. Meyer, Phys. Rev. Lett. 111, 243002 (2013).

[21] M. Hentschel, R. Kienberger, C. Spielmann, G. A. Reider, N. Milosevic, T. Brabec, P. Corkum, U. Heinzmann, M. Drescher, and F. Krausz, Nature (London) 414, 509 (2001).

[22] M. Drescher, M. Hentschel, R. Kienberger, M. Uiberacker, V. Yakovlev, A. Scrinzi, T. Westerwalbesloh, U. Kleineberg, U. Heinzmann, and F. Krausz, Nature (London) 419, 803 (2002).

[23] R. Kienberger, E. Goulielmakis, M. Uiberacker, A. Baltuska, V. Yakovlev, F. Bammer, A. Scrinzi, T. Westerwalbesloh, U. Kleineberg, U. Heinzmann, M. Drescher, and F. Krausz, Nature (London) 427, 817 (2004).

[24] F. Krausz and M. Y. Ivanov, Rev. Mod. Phys. 81, 163 (2009).

[25] J. M. Bizau, J.-P. Champeaux, D. Cubaynes, F. J. Wuilleumier, F. Folkmann, T. S. Jacobsen, F. Penent, C. Blancard, and H. Kjeldsen, Astron. Astrophys. 439, 387 (2005).

[26] P. G. Burke and K. T. Taylor, J. Phys. B: At. Mol. Phys. 8, 2620 (1975).

[27] K. A. Berrington, W. B. Eissner, and P. H. Norrington, Computer Physics Communications 92, 290 (1995).

[28] M. Seaton, J. Phys. B: At. Mol. Phys. 20, 6363 (1987).

[29] K. A. Berrington, P. G. Burke, K. Butler, M. J. Seaton, P. J. Storey, K. T. Taylor, and Y. Yan, J. Phys. B: At. Mol. Phys. 20, 6379 (1999).

[30] J. A. Fernley, K. T. Taylor, and M. J. Seaton, J. Phys. B: At. Mol. Phys. 20, 6457 (1987).

[31] D. A. Verner, G. J. Ferland, K. T. Korista, and D. G. Yakovlev The Astrophysical Journal 465, 487 (1996).

[32] The Opacity Project Team, The Opacity Project Vol. 1 (Institute of Physics Publications, Bristol, UK, 1995).

[33] K. P. Dere, E. Landi, H. E. Mason, B. C. Monsignori Fossi, and P. R. Young, Astron. Astrophys. Suppl. Ser. 125, 149 (1997).

[34] E. Landi, P. R. Young, K. P. Dere, G. Del Zanna, and H. E. Mason, Astrophys. J 763, 86 (2013).

[35] O. Zatsarinny and C. Froese Fischer, J. Phys. B: At. Mol. Opt. Phys. 33, 313 (2000).

[36] O. Zatsarinny, Comp. Phys. Commun. 174, 273 (2006).

[37] O. Zatsarinny and C. Froese Fischer, Comp. Phys. Commun. 180, 2041 (2009).

[38] O. Zatsarinny and K. Bartschat, J. Phys. B: At. Mol. Opt. Phys. 46, 112001 (2013).

[39] C. Froese Fischer, Comp. Phys. Commun. 128, 635 (2000).
[40] C. Froese Fischer, G. Tachiev, G. Gaigalas, and M. R. Godefroid, Comp. Phys. Commun. 176, 559 (2007).

[41] T. Carette, J. M. Dahlström, L. Argenti, and E. Lindroth, Phys. Rev. A 87, 023420 (2013).

[42] Z. Sibincic, Phys. Rev. A 5, 1150 (1972).

[43] D. Goorvitch and F. P. J. Valero, Astrophys. J 171, 643 (1972).

[44] C. M. Brown, S. G. Tilford, and M. L. Ginter, J. Opt. Soc. Am. 64, 877 (1974).

[45] G. A. Odintzova and A. R. Stringanov, J. Phys. Chem. Ref. Data 8, 63 (1979).

[46] G. Merkelis, M. J. Vilkas, G. Gaigalas, and R. Kisielius, Phys. Scr. 51, 233 (1995).

[47] J. Olsen, M. R. Godefroid, P. Jönsson, P. A. Malmqvist, and C. F. Fischer, Phys. Rev. E 52, 4499 (1995).

[48] M. R. Godefroid, C. F. Fischer, and P. Jönsson, Phys. Scr. T 65, 70 (1996).

[49] G. Tachiev and C. Froese Fischer, J. Phys. B: At. Mol. Opt. Phys. 33, 2419 (2000).

[50] H. Lundberg, Z. S. Li, and P. Jönsson, Phys. Rev. A 63, 032505 (2001).

[51] W. L. Glab and A. M. Falleur, J. Quant. Spectrosc. Rad. Transf. 73, 121 (2002).

[52] L. Liang and Y. C. Wang, J. Phys. B: At. Mol. Opt. Phys. 36, 4387 (2003).

[53] C. a. Nicolaides, Int. J. Quant. Chem. 102, 250 (2005).

[54] F. J. Gálvez, E. Buendía, and A. Sarsa, J. Chem. Phys. 123, 034302 (2005).

[55] U. I. Safronova, J. Plasma Fusion Res. SERIES 7, 282 (2006).

[56] Z. Tian-yi and Z. Neng-wu, Acta Phys. Polon. A 116, 141 (2009).

[57] C. X. Almora-Díaz and C. F. Bunge, Int. J. Quant. Chem. 110, 2982 (2010).

[58] M. B. Ruiz, M. Rojas, G. Chicón, and P. Otto, Int. J. Quant. Chem. 111, 1921 (2011).

[59] S. Bubin and L. Adamowicz, Phys. Rev. A 83, 022505 (2011).

[60] C. Froese Fischer, A. Ynnerman, and G. Gaigalas, Phys. Rev. A 51, 4611 (1995).

[61] C. Froese Fischer and G. Gaigalas, J. Phys. B: At. Mol. Opt. Phys. 29, 1169 (1996).

[62] P. J. Marchalant and K. Bartschat, J. Phys. B: At. Mol. Opt. Phys. 30, 4373 (1997).

[63] Y.-K. Kim and P. M. Stone, Phys. Rev. A 64, 052707 (2001).

[64] C. P. Ballance, D. C. Griffin, K. a. Berrington, and N. R. Badnell, J. Phys. B: At. Mol. Opt. Phys. 40, 1131 (2007).

[65] J. C. Berengut, S. D. Loch, M. S. Pindzola, C. P. Ballance, and D. C. Griffin, Phys. Rev. A 76, 042704 (2007).

[66] M. R. Talukder, Appl. Phys. B 93, 567 (2008).

[67] C. Chen, Y. Sun, and B. Cong Gou, J. Quant. Spectrosc. Rad. Transf. 145, 1 (2014).

[68] N. R. Badnell, D. Petrini, and S. Stoica, J. Phys. B: At. Mol. Opt. Phys. 30, L665 (1999).

[69] W. G. Lynam, P. K. Carroll, J. T. Costello, D. Evans, and G. O'Sullivan, J. Phys. B: At. Mol. Opt. Phys. 25, 3963 (1999).

[70] U. Litzén, T. Zethson, P. Jönsson, J. Kasten, R. Kling, and F. Launay, Phys. Rev. A 57, 2477 (1998).

[71] S. Schippers, A. Müller, B. M. McLaughlin, A. Aguilar, C. Cisneros, E. D. Emmons, M. F. Gharaibeh, and R. A. Phaneuf, J. Phys. B: At. Mol. Opt. Phys. 36, 3371 (2003). 
[72] I. Martinson, Y. Awaya, J. O. Ekberg, I. Kink, S. Mannervik, and A. N. Ryabtsev, J. Phys. B: At. Mol. Opt. Phys. 36, 419 (2003).

[73] D.-S. Kim and S. T. Manson, J. Phys. B: At. Mol. Opt. Phys. 37, 4013 (2004).

[74] A. N. Ryabtsev, I. Kink, Y. Awaya, J. O. Ekberg, S. Mannervik, A. Ölme, and I. Martinson, Phys. Scr. 71, 489 (2005).

[75] J.-T. Hsiao, H.-L. Sun, S.-F. Lin, and K.-N. Huang, J. At. Mol. Opt. Phys. 2011, 452026 (2011).

[76] A. Kupliauskiene, Phys. Scr. 55, 445 (1997).

[77] A. M. Boesgaard, E. J. McGrath, D. L. Lambert, and K. Cunha, Astrophys. J 606, 306 (2004).

[78] F. Primas, Proc. Int. Astron. Union 5, 221 (2009).

[79] S. G. Johansson, U. Litzen, J. Kasten, and M. Kock, Astrophys. J 403, L25 (1993).

[80] G. Tanner, K. Richter, and J.-M. Rost, Rev. Mod. Phys. 72, 497 (2000).

[81] J. L. Sanz-Vicario, J. C. Cardona, and E. Lindroth, Phys. Rev. A 78, 053411 (2008).

[82] L. Argenti and R. Moccia, J. Phys. B: At. Mol. Opt. Phys. 39, 2773 (2006).

[83] L. Argenti and E. Lindroth, Phys. Rev. Lett. 105, 053002 (2010).

[84] L. Argenti, R. Pazourek, J. Feist, S. Nagele, M. Liertzer, E. Persson, J. Burgdörfer, and E. Lindroth, Phys. Rev. A 87, 053405 (2013).

[85] Á. Jiménez Galán, L. Argenti, and F. Martín, New J. Phys. 15, 113009 (2013).

[86] Á. Jiménez-Galán, L. Argenti, and F. Martín, Phys. Rev. Lett. 113, 263001 (2014).

[87] S. Heuser, Á. Jiménez-Galán, C. Cirelli, M. Sabbar, R. Boge, M. Lucchini, L. Gallmann, I. Ivanov, A. S. Kheifets, M. J. Dahlström, E. Lindroth, L. Argenti, F. Martín, and U. Keller, arXiv:1503.08966.

[88] Á. Jiménez Galán, F. Martín, and L. Argenti, Phys. Rev. A 93, 023429 (2016).

[89] R. G. Newton, Scattering Theory of Waves and Particles, 2nd ed. (Springer-Verlag, New York, 1982).

[90] J. C. Cardona, J. L. Sanz-Vicario, and F. Martín, Phys. Rev. A 82, 022501 (2010).

[91] I. Cacelli, V. Carravetta, and R. Moccia, J. Chem. Phys. 85, 7038 (1986).

[92] R. Moccia and P. Spizzo, Phys. Rev. A 43, 2199 (1991).

[93] I. Cacelli, V. Carravetta, A. Rizzo, and R. Moccia, Phys. Rep. 205, 283 (1991).

[94] L. Argenti and R. Moccia, J. Phys. B: At. Mol. Opt. Phys. 41, 035002 (2008).

[95] E. Lindroth and L. Argenti, Adv. Quantum Chem. 63, 247 (2012).

[96] J. Taylor, Scattering Theory: The Quantum Theory on Nonrelativistic Collisions (Wiley \& Sons, New York, 1972).
[97] C. Bottcher and A. Dalgarno, Proc. Phys. Soc. A 340, 187 (1974).

[98] W. Müller and W. Meyer, J. Chem. Phys. 80, 3311 (1984).

[99] R. Moccia and P. Spizzo, Phys. Rev. A 39, 3855 (1989).

[100] E. L. Shirley, X. Zhu, and S. G. Louie, Phys. Rev. B 56, 6648 (1997).

[101] M. Kutzner, J. A. Robertson, and P. Pelley, Phys. Rev. A 62, 062717 (2000).

[102] V. K. Voora, J. Ding, T. Sommerfeld, and K. D. Jordan, J. Phys. Chem. B 117, 4365 (2013).

[103] S. Mengali and R. Moccia, J. Phys. B: At. Mol. Opt. Phys. 29, 1597 (1996).

[104] H. Bachau, E. Cormier, P. Decleva, J. E. Hansen, and F. Martín, Rep. Prog. Phys. 64, 1815 (2001).

[105] L. Argenti and R. Colle, Comp. Phys. Commun. 180, 1442 (2009).

[106] M. Hamermesh, Group Theory and Its Application to Physical Problems, 2nd ed. (Addison-Wesley, Reading, MA, 1964).

[107] R. McWeeny, Methods of Molecular Quantum Mechanics, 2nd ed. (Academic Press, London, 1992).

[108] A. Kramida, Y. Ralchenko, J. Reader, and N. A. Team, NIST Atomic Spectra Database (version 5.2) (2014); A. E. Kramida and A. N. Ryabtsev, Phys. Scr. 76, 544 (2007).

[109] E. R. Davidson, S. A. Hagstrom, S. J. Chakravorty, V. M. Umar, and C. F. Fischer, Phys. Rev. A 44, 7071 (1991).

[110] J. Carlsson, P. Jönsson, L. Sturesson, and C. Froese Fischer, Phys. Rev. A 49, 3426 (1994).

[111] P. Jönsson, C. F. Fischer, and M. R. Godefroid, J. Phys. B: At. Mol. Opt. Phys. 29, 2393 (1996).

[112] J. A. Fernley, A. Hibbert, A. E. Kingston, and M. J. Seaton, J. Phys. B: At. Mol. Opt. Phys. 32, 5507 (1999).

[113] F. J. Gálvez, E. Buendía, and A. Sarsa, J. Chem. Phys. 122, 154307 (2005).

[114] H. Gharibnejad and A. Derevianko, Phys. Rev. A 86, 022505 (2012).

[115] C. Froese Fischer, S. Verdebout, M. R. Godefroid, P. Rynkun, P. Jönsson, and G. Gaigalas, Phys. Rev. A 88, 062506 (2013).

[116] J. R. Fuhr and W. L. Wiese, J. Phys. Chem. Ref. Data 39, 013101 (2010).

[117] See Supplemental Material at http://link.aps.org/supplemental/ 10.1103/PhysRevA.93.042503 for more values from some of the tables.

[118] H. Friedrich, Theoretical Atomic Physics (Springer-Verlag, Heidelberg, 1990).

[119] U. Fano, Phys. Rev. 124, 1866 (1961).

[120] R. A. Roig and G. Tondello, J. Phys. B: At. Mol. Phys. 9, 2373 (1976).

[121] D. A. Varshalovich, A. N. Moskalev, and V. K. Kersonskii, Quantum Theory of Angular Momentum (World Scientific, Singapore, 1984), p. 514. 